\title{
Untersuchungen über die Auswirkung kommunaler Abwässer auf das benthische Ökosystem mariner Watten*
}

\author{
G. OTTE** \\ Institut für Hydrobiologie und Fischereiwissenschaften der Universität Hamburg, \\ Palmaille 55, D-2000 Hamburg 50, Bundesrepublik Deutschland
}

\begin{abstract}
Investigations on the effects of domestic sewage on the benthic ecosystem of marine intertidal flats. The influence of domestic sewage on intertidal sand and mud flat benthic communities has been studied at three minor outfalls around the North Sea island of Sylt (F. R. Germany) and at the main outfall of Vancouver, B. C. (Canada). During high tide, domestic waste water was found at the water surface due to its lower specific gravity. Consequently, $\mathrm{O}_{2}$ depletion and salinity decrease occured mainly in surface waters. Oxy g e $\mathrm{def}$ i c i e n cy prevailed in the sediment-water layer, when settled sewage particles were re-suspended by strong tidal currents. This resulted in anaerobic conditions at the sediment surface for up to $40 \%$ of the day. Substantial pH decrease and increase in seston load of the water were observed only along the sewage channels and around the outlets when large quantities of sewage were discharged. The amount of particulate organic material increased up to $10 \%$ and more in muddy sediments due to sedimentation of sewage sludge. Considerable e u troph ic a t i o $\mathrm{n}$ of the mud flats resulted from nutrient load of the sewage and from decomposition of faecal material deposited on the sediments. The vertical distribution of microalgae in the sediment was not influenced by sewage. Primary productivity of microalgae on the sediment surface was positively correlated with the degree of pollution. In heavily and mediumly polluted zones, heterotrophic bacterial production exceeded primary production rates at the sediment surface. Compared with unpolluted intertidal flats the $\mathrm{number}$ of $\mathrm{macrobenthic}$ $\mathrm{speci}$ e $\mathrm{s}$ was not reduced in polluted mud flats. In regard to overall species abundance, the macrofauna associations of the disposal area showed three distinct zones: (1) Heavy pollution around the outfall resulted in a degradation zone either without benthic invertebrates or with a very low density of individuals; (2) medium polluted areas were characterized by a zone of maximum density; (3) with decreasing or diminishing pollution the number of individuals returned to normal values ("background values"). The distribution of species within these zones indicated a significant difference between the response of the "channel fauna" and the "flat fauna" to pollution: while the number of species increased continously inside the channels with decreasing pollution, the species variety of the tidal flats was positively correlated with the number of individuals. The dive r s it y of the $\mathrm{b}$ e $\mathrm{nth}$ os inside the channels was negatively correlated with the degree of pollution, whereas the diversity indices for the fauna on the tidal flats increased quickly from the centre of pollution, remained at a high level in the medium polluted zone, and decreased again to the area of diminishing pollution. The $\mathrm{number}$ of $\mathrm{dominating} \mathrm{species}$ in the sewage channel increased from the outlet towards the low-tide level, with ratios of 0.9 to 0.33 ; on the flats the number remained constant throughout the three zones, with maximum ratios of 0.5 , demonstrating that no qualitative
\end{abstract}

\footnotetext{
* Dissertation aus dem Fachbereich Biologie der Universität Hamburg.

$*$ Neue Anschrift: Biologische Anstalt Helgoland (Zentrale), Palmaille 9, D-2000 Hamburg 50.
} 
changes for the macrobenthos of intertidal flats result from domestic sewage disposals. In polluted intertidal flats the macrobenthos is dominated by muddy-sand species whose adaptability to varying sedimentary factors, such as grain size distribution and content of organic material (coefficients of variability: 50.7 and $45.7 \%$, respectively), is relatively high compared with mud and sand species. A special indicator community for polluted intertidal areas does not exist. Intensified waste-water treatment prior to discharge reduced the size of the degradation zone around the outfalls while it simultaneously caused a lower density of the macrobenthos in the medium polluted zone.

\section{EINLEITUNG}

Wattengebiete dienen den Küstenmeeren als natürliche Ablagerungs- und Faulräume für Schwebstoffe. Besonders deutlich läßt sich dies im Mündungsgebiet von Flüssen zeigen, wo dem Festland breite Schlickwattengürtel als Auffanggebiete für den ins Meer eingebrachten Detritus natürlichen und anthropogenen Ursprungs vorgelagert sind (Caspers, 1968). Unter günstigen Sauerstoffverhältnissen werden die partikulären organischen Substanzen hier der weiteren Dekomposition zugeführt oder bei zu starker Aufschlickung dem Stoffkreislauf im Meer durch einen Verlandungsprozeß zum Teil entzogen.

Wattengebiete sind allgemein an einen hohen Import abbaubaren Restmaterials adaptiert. Die Produktionskraft solcher Gebiete zeigt, daß das hohe trophische Niveau vom gesamten "Ókosystem Schlickwatt" genutzt werden kann (Wohlenberg, 1937; Dörjes, 1970; Hargrave, 1972; Cadée \& Hegemann, 1974).

Die Frage ist, wie Schlickwatten bei einer zusätzlichen, künstlichen Erhöhung ihrer Trophie und Saprobität durch das Einleiten von organischen Abwässern reagieren, ob (1) dieses an die Oxydation großer Detritusmengen adaptierte System zusammenbricht, (2) es keine meßbare Reaktion zeigt oder (3) durch solchen zusätzlichen Input von Energie eine Biostimulation dieser schon hochproduktiven Meeresgebiete möglich ist.

Uber die Auswirkungen von sublitoralen Abwassereinleitungen ins Meer sind schon intensive Untersuchungen durchgeführt worden (Wilhelmi, 1915 a, b; Reish, 1957; Felice, 1959; Croft, 1960; Young, 1964; Olsen \& Burgess, 1967; Beyer, 1968; Tulkki, 1969; O'Sullivan, 1971; Borowitzka, 1972; Anderson \& Read, 1974 u. a.). Auch ist vielfach versucht worden, die im Süßwasser entwickelte Systematik für Indikatorarten und -gesellschaften (Kolkwitz \& Marson, 1908, 1909; Liebmann, 1947, 1960) auf marine Biotope mit unterschiedlichen Verschmutzungsstufen zu übertragen (Nakajima, 1958; Kolt \& Ben Ari, 1968; Akesson, 1970; Reish, 1960, 1972; Oglesby \& Jamison, 1968; Tulkki, 1969).

Über den direkten Einfluß von organischen Abwässern auf Watten-Lebensgemeinschaften ist nur wenig bekannt (Oglesby \& Jamison, 1968; Perkins \& Abbott, 1972; BC Research, 1973, 1974), obwohl zu vermuten war, daß wegen der hydrographischen Bedingungen die Aufnahmepotenz von Watten für fäulnisfähige Stoffe wesentlich größer sein muß als die limnischer Gewässer oder mariner sublitoraler Schlickgebiete: der tidenbedingte kontinuierliche Wasserwechsel sorgt für ein hohes $\mathrm{O}_{2}$-Angebot an der Sedimentoberfläche, das beim Trockenfallen der Wattflächen noch verbessert wird. Zudem verhindern Turbulenzen und Tidenströmungen eine Schichtung des Wasserkörpers.

Die vorliegende Arbeit betrifft die Frage, in welchem Ausmaß eine Abwassereinleitung in das "Okosystem Wattenmeer" die Lebensgemeinschaften über die natürlichen Fluktuationen hinaus beeinflußt. $\mathrm{Da}$ Veränderungen einzelner Umweltfaktoren Auswirkungen auf das Gesamtsystem haben, wurden möglichst viele abiotische und biotische 
Faktoren quantitativ erfaßt. Hierzu gehören Untersuchungen zur Hydrochemie des Wasserkörpers und des Sedimentes, zum Sedimentgefüge und zur -morphometrie der Untersuchungsgebiete. Ferner sind Analysen der stoffwechseldynamischen Umsatzprozesse in den Sedimenten und der Struktur der Makrobenthos-Assoziationen in den Zonen unterschiedlicher Belastungen essentieller Bestandteil zur Abschätzung der Folgen einer künstlichen Trophie- und Saprobitätserhöhung in Wattengebieten.

Die Untersuchungen wurden von 1970 bis 1974 in den Wattengebieten bei Sylt (Einleitungsbereiche der Gemeinden Kampen, List, Braderup) sowie in einem Wattenabschnitt des Fraser River-Ästuars (Einleitungsbereich der Stadt Vancouver/Canada) durchgeführt.

\section{PROBLEMATIK EINER ABWASSEREINLEITUNG IN WATTENGEBIETE}

Die Zeit geringer Strömungsgeschwindigkeiten, die eine verstärkte Sedimentation erlaubt, ist bei Hochwasser länger als bei Niedrigwasser. Da die Wassermassen sich zur Hochwasserzeit am weitesten landwärts befinden, dienen die Schlickwattengebiete als Ablagerungsräume für das mitgeführte Seston (Postma, 1954, 1960; Reineck, 1970; DeJonge \& Postma, 1974).

Die organischen Bestandteile des Sestons - im holländischen Wattenmeer durchschnittlich $13 \%$ (Postma, 1954) - werden hier der Dekomposition zugeführt. Die dabei anfallenden Nährstoffe (z. B. Phosphat und Nitrat) gehen zum Teil in Lösung und werden mit den Tiden verdriftet. Die Mineralisation und die Freisetzung der Endprodukte aus dem Sediment wird durch Benthosorganismen gefördert (Hecht, 1933; van Straaten, 1954; Lillelund, 1969). Ein anderer Teil der Mineralien wird an Ort und Stelle von benthischen Mikroalgen und Seegräsern aufgenommen. Ausgedehnte Seegraswiesen in Watten weisen auf einen Eutrophierungseffekt hin. Durch den Import von fäulnisfähigen Stoffen und den hohen Sauerstoffeinträgen durch die Tiden kommt es in diesen "Faulräumen des Meeres" zu einer gesteigerten mikrobiellen Aktivität. Wegen des darauffolgenden Exportes der Abbauprodukte lassen sich von den Schlickwatten im Prinzip Parallelen zu biologischen Abwasserreinigungsanlagen ziehen. Die Kapazitäten solcher natürlichen Selbstreinigungsräume des Meeres werden begrenzt durch die Diffusionsgeschwindigkeit bzw. Eindringtiefe des Sauerstoffs ins Sediment; je feiner das sedimentierte Seston und je höher sein Anteil an abbaubarem organischen Material, desto höher ist die Bioaktivität im Sediment, die nachgewiesen werden kann durch erhöhte Respirationsraten und höhere Keimzahlen (Krumbein, 1971; Hargrave, 1972; Bianchi, 1973; Dale, 1974). Das führt zu gesteigertem Sauerstoffverbrauch im Sediment, wodurch die Eindringtiefe des Sauerstoffs abnimmt; er wird schon in den oberen Schichten vollständig aufgebraucht.

Ist die Sedimentationsrate so hoch, daß der aerobe, mikrobielle Abbau der organischen Substanzen nicht mehr gewährleistet ist, kommt es im Sediment schon unter der Oberflächenschicht zu anaeroben Verhältnissen. Dabei wird der noch unvollständig abgebaute Detritus kontinuierlich von neuem, sedimentierenden Detritus überlagert. Der weitere Abbau muß also anaerob stattfinden. Er führt nicht zur vollständigen Mineralisation. Dadurch werden dem Meer laufend Nährstoffe entzogen.

Sekundäre Verschmutzungseffekte, die aus einer rasanten Eutrophierung resultieren 
würden, bleiben daher begrenzt. Als sichtbarer Effekt kann lediglich eine starke Aufschlikkung der Wattflächen beobachtet werden.

Durch den von Ebbe und Flut bedingten Wasseraustausch und den Zutritt der Luft zur Sedimentoberfläche kommt es unter natürlichen Bedingungen nur äußerst selten zu einem vollständigen Fehlen von Sauerstoff auf dem Watt. Zumindest die Sedimentoberfläche ist fast ausnahmslos dem oxydativen Abbau zugänglich. Dies ist für das Makrobenthos entscheidend, da die meisten Arten aus der Wasser-Sediment-Grenzschicht ihren Sauerstoffbedarf decken (Friedrich, 1965; Dörjes, 1970; Newell, 1970).

Bei einer Einleitung von häuslichen Abwässern in Wattengebiete findet eine Erhöhung der Saprobität und der Trophie statt. Durch die zusätzliche Zufuhr von organischen, abbaubaren Substanzen muß also eine verstärkte mikrobielle Aktivität einsetzen (soweit die $\mathrm{O}_{2}$-Verhältnisse noch einen aeroben Abbau zulassen).

Ferner wird bei der Einleitung von im Abwasser vorhandenen eutrophierenden Mineralien sowie durch deren Freisetzung aus Dekompositionsprozessen im Sediment eine Förderung der Primärproduktion der benthischen Mikroalgen zu erwarten sein. Beide Komponenten - die erhöhte mikrobielle Aktivität und die Steigerung der Primärproduktion - bedeuten für Detritusfresser, also den Hauptanteil des in Schlickwatten lebenden Makrobenthos, ein gesteigertes Nahrungsangebot.

Eine solche Biostimulation auf den verschiedenen Stufen der Nahrungsketten wird jedoch nur festzustellen sein, solange es nicht zu Belastungen kommt, die zu einem "Umkippen" des gesamten Systems Schlickwatt führen. Man kann allerdings davon ausgehen, daß die Pufferungskapazität der Watten für extreme Umwelteinflüsse wegen ihrer günstigen hydrographischen Verhältnisse und der Prädisposition für die Verarbeitung organischen Restmaterials (s. o.) größer ist als die anderer aquatischer Biotope.

\section{MATERIAL UND METHODEN}

\section{Untersuchungsgebiete}

Nur wenige Städte und Siedlungen leiten ihre Abwässer in Wattengebiete. An der deutschen Nordseeküste sind es vor allem die Inselgemeinden, die die Watten als Vorfluter nutzen. Die seeseitigen Strände bleiben dem Tourismus vorbehalten.

Für die vorliegenden Untersuchungen wurden die Abwassereinleitungsstellen von Kampen, List und Braderup auf Sylt ausgewählt (Abb. 1). Allerdings sind die hier anfallenden Abwassermengen gering und - bedingt durch den Tourismus - starken saisonalen Schwankungen unterworfen. Es war daher sinnvoll, vergleichende Untersuchungen in einem Wattengebiet durchzuführen, das von einer Großstadt als Vorfluter benutzt wird. Dazu bot sich ein Abschnitt des Fraser River Ästuars an, in das die Stadt Vancouver/Canada ihre Abwässer einleitet (Abb. 2).

\section{Sylt}

Die Kläranlagen der drei Gemeinden Kampen, List und Braderup entwässern in ein ausgedehntes, überwiegend schlickiges Wattengebiet, das im Osten vom schleswig-holstei- 
nischen Festland, im Süden vom Inseldamm und im Westen vom Nordteil der Insel Sylt begrenzt wird. Der am Damm noch sehr schlickige und breite Wattstreifen mit geringem Gefälle geht in Richtung List in ein festeres Feinsandwatt über. An der Nordspitze der Insel wird dieses durch ein Grobsandwatt mit abnehmender Flächenausdehnung und daher stärkerem Gefälle abgelöst.

Das Schwergewicht der Untersuchungen im Sylter Wattenmeer wurde auf den Einflußbereich der Kampener Abwässer gelegt, da hier (1) die größte Abwassermenge eingeleitet wird, und (2) das Watt sehr homogen und übersichtlich ist. Daher ist ein Abwassereinfluß hier am besten zu erfassen. Nachfolgend werden die drei Untersuchungsgebiete wegen ihrer unterschiedlichen Lage innerhalb des Wattenstreifens getrennt beschrieben.

K a m p e n : Etwa 2 Kilometer nördlich vom Ort Kampen liegt die Kläranlage der Gemeinde. Ihr Abfluß führt über einen Wiesenentwässerungsgraben in eine offene, von Vorlandwiesen begrenzte Bucht des an dieser Stelle rund $800 \mathrm{~m}$ breiten Wattenstreifens; nach der kleiig-sandigen Abbruchkante der Wiesen beginnt ein von Queller (Salicornia europaea) bewachsener, nur mäßig verschlickter Feinsandstreifen, der nach ungefähr $30 \mathrm{~m}$ in eine stärker verschlickte Faulschlammzone übergeht. Dieser Streifen ist nicht bewachsen. Nach weiteren $150 \mathrm{~m}$ beginnen ausgedehnte, dichte Seegraswiesen, die von Zostera nana gebildet werden und sich fast bis zur Niedrigwasserlinie erstrecken. Dort lockern sie sich auf und werden von einzelnen Gruppen einer anderen Seegras-Art (Zostera angustifolia) abgelöst. Im Bereich der Seegraswiesen findet man weichen, tiefen Schlick, da das dichtstehende Seegras als Sestonfalle wirkt (Wohlenberg, 1937). Durch das geringe Gefälle existiert keine ausgeprägte Tiden-Niedrigwasserlinie. Die Breite der trockenfallenden Zone ist daher stark vom Wasserstand bei Niedrigwasser (TNW) abhängig. Im Bereich dieser variablen TNW-Linie beginnen große Mytilus-Bänke, die kommerziell genutzt werden. Der flache Abwasserpriel durchschneidet die unbewachsenen Wattflächen und die Seegraswiesen etwa senkrecht zum Ufer. Entlang der Prielränder wird das Seegras zurückgedrängt. Die Stationen, von denen Proben für die biologischen und chemisch-physikalischen Analysen entnommen wurden, lagen auf drei Transekten: neun Stationen von der Einleitungsstelle bis zur Niedrigwasserlinie jeweils im bzw. am Rande des Abwasserprieles (K-Transekt) und je sechs Stationen nach Norden (L-Transekt) und Süden (M-Transekt) vom Priel in einem Abstand von etwa $30 \mathrm{~m}$ parallel zum Ufer (Abb. 1).

L i s $t$ : Die Abwässer der Gemeinde List fließen bei Hochwasser von der Kläranlage in einen lagunenartigen Binnensee und mischen sich hier mit Süßwasser aus Wiesengräben sowie mit dem nachströmenden Seewasser. Bei Niedrigwasser läuft das Abwasser in eine Bucht, die durch eine hakenförmige Sanddüne gebildet wird, und weiter über ausgedehnte Muschelbänke mit dem Haupttidenstrom in die offene Nordsee. Die Sedimente der maximal $400 \mathrm{~m}$ breiten Bucht bestehen überwiegend aus Grobsand, sind jedoch mit einer feinen Schlickschicht überzogen. Wegen des hohen Wassergehaltes ist der Boden der Mulde sehr weich. Bei Niedrigwasser bleibt an der tiefsten Stelle ein Wattentümpel zurück. Die drei Probenstationen lagen $10 \mathrm{~m}, 50 \mathrm{~m}$ und $80 \mathrm{~m}$ von der Einleitungsstelle entfernt auf einer geraden Linie, parallel zu dem bei Ebbe abfließenden Abwasser.

B r a de r u p : Das Abwasser fließt hier vom Klärwerk durch einen unterirdischen Düker ab und tritt rund $30 \mathrm{~m}$ vom Ufer entfernt - bei Niedrigwasser springbrunnenartig aus einem Steinhaufen im Watt wieder zutage. Es läuft dann nach $300 \mathrm{~m}$ bis $400 \mathrm{~m}$ über das 
sehr weiche, schlickige Sediment in einen breiten Priel. Die Schlickflächen sind im Sommer dicht mit Zostera bewachsen, mit Ausnahme des unmittelbaren Einleitungsbereiches und des Streifens, über dem bei Niedrigwasser das Abwasser abfließt. In diesem Untersuchungsgebiet wurden Proben von drei Stationen im Abstand von $10 \mathrm{~m}$ um den Auslauf herum entnommen.

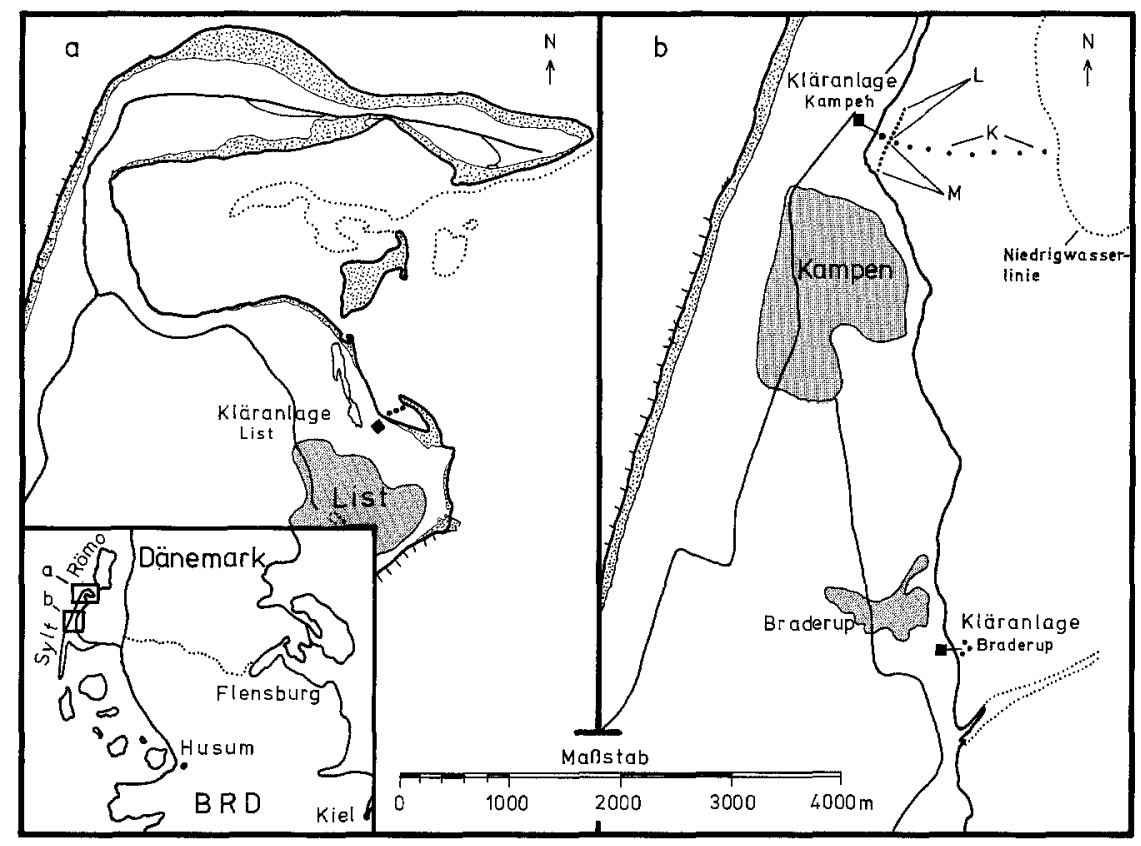

Abb. 1: Untersuchungsgebiete im Sylter Wattenmeer. Eingetragen sind die Punkte der Probenentnahme und die Meßstationen. Transektbezeichnungen: L = Kampen Nord (im Text L1-L6, von Süd nach Nord); $\mathrm{M}=$ Kampen Süd (im Text M1-M6, von Nord nach Süd); $\mathrm{K}=$ Abwasserpriel (im Text KO-K12, von West nach Ost). Links unten: Ubersichtsskizze für die Lage der dargestellten Kartenausschnitte

\section{Vancouver}

Vancouver liegt im Mündungsdelta des Fraser Rivers, der in die Strait of Georgia mündet. Im Bereich des Deltas hat der Fluß weite Wattflächen an der sonst überwiegend felsigen Pazifik-Küste aufgeschwemmt. In dieses Watt leitet die Stadt Voncouver ihre Abwässer (Abb. 2).

Das Einleitungsgebiet wird im Norden durch eine bis zur Niedrigwasserlinie reichende Mole begrenzt. Sie verhindert, daß das Abwasser an die Badestrände der Stadt gelangt.

Im Süden bilden eine kurze Mole für Navigationslichter des Flughafens und der Mittelarm des Fraser Rivers die Grenze. Zur Küste hin geht das Watt durch einen $50 \mathrm{~m}$ bis 
$100 \mathrm{~m}$ breiten Streifen mit üppig wuchernden halophilen Kormophyten in Festland über. Vom Ufer bis zur Niedrigwasserlinie beträgt die Entfernung rund 5,8 km, während das Watt zwischen der Nordmole und dem Mittelarm des Fraser Rivers rund 2,8 km breit ist. Das gesamte Untersuchungsgebiet hat damit eine Ausdehnung von etwa $16,2 \mathrm{~km}^{2}$.

Das Watt fällt nur langsam zur Niedrigwasserlinie hin ab und ist im allgemeinen ohne deutliche Konturen. Es wird lediglich vom künstlichen Abwasserkanal und von einem natürlichen Priel durchschnitten, der in der Mitte des Wattstreifens aus den Salzwiesen kommt und zunächst seewärts fließt. Dann wendet er sich nach Norden und mündet im Abwasserkanal. Das Abwasser wird über eine Kaskade am landseitigen Ende der Nordmole ins Watt eingeleitet und fließt bei Niedrigwasser in dem künstlichen, $1 \mathrm{~m}$ bis $3 \mathrm{~m}$ tiefen Kanal im Abstand von ungefähr $50 \mathrm{~m}$ parallel zur Mole in die offene See. Das in Küstennähe sehr schlickige Sediment wird seewärts kontinuierlich sandiger. Das weichste Sediment fand sich um die Einleitungsstelle herum. Auch im Abwasserkanal wurde teilweise sehr weiches Sediment festgestellt. Zur Niedrigwasserlinie überwiegen dagegen reine Sandgebiete.

Die Sedimentoberfläche war im Sommerhalbjahr mit Diatomeen bedeckt, mit Ausnahme des Gebietes um die Einleitungsstelle und auf beiden Seiten des oberen Abwasser-

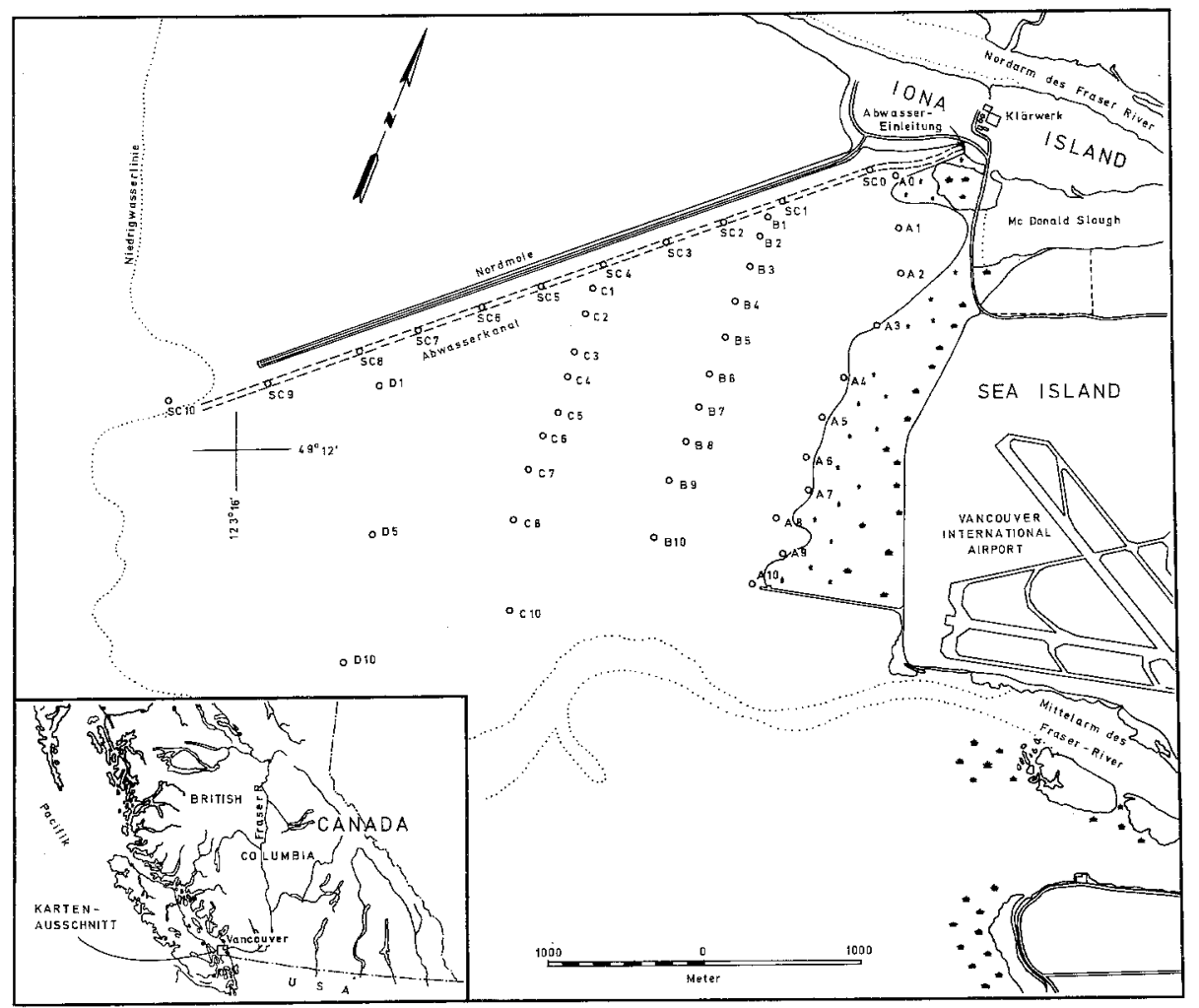

Abb. 2: Untersuchungsgebiet bei Vancouver mit den Positionen der Probenstationen. Links unten: Ubersichtskarte und Lage des Kartenausschnittes 
kanals. In diesem Gebiet konnten dichte Polster von gelb-grünen Cyanophyceen beobachtet werden. Im gesamten Untersuchungsgebiet wurde kein Seegras gefunden, obwohl es auf benachbarten Wattenabschnitten teilweise recht häufig vorkam. Vermutlich ist der Süßwassereinfluß des Fraser Rivers für das Fehlen verantwortlich (mündl. Mitt. von Dr. Levings, Pacific Environment Institute, Vancouver).

Die Probenstationen lagen auf fünf Transekten, wovon der A-, B-, C- und DTransekt auf dem Watt verliefen und der SC-Transekt im Abwasserkanal lag (Abb. 2). Die Stationen jedes einzelnen Transektes wurden mit Hilfe einer Höhenkarte (Swan Wooster, 1967) so ausgewählt, daß sie etwa die gleichen Wasserbedeckungszeiten aufwiesen. Die Stationen des D-Transektes fielen nicht bei jeder Tide trocken, da die kanadische PazifikKüste in einem Gebiet mit gemischten Tiden liegt, deren Amplitude einen Tages- und einen Zwei-Wochen-Zyklus aufweist.

\section{Feld- und Laboruntersuchungen}

In den Sylter Wattengebieten wurden die Untersuchungsstationen mit einem Meßrad eingemessen und mit Eisenstangen markiert. Alle Stationen konnten bequem zu Fuß erreicht werden.

Im Untersuchungsgebiet bei Vancouver dagegen war das Watt teilweise nicht begehbar. Darüber hinaus waren die Entfernungen zu einigen Stationen von Land aus zu groß, um sie während der Niedrigwasserzeit ohne Fahrzeug zu erreichen. Die Stationen des Aund D-Transektes sowie die am weitesten seewärts liegenden Stationen des C-Transektes sind daher mit einem mit Radar ausgerüsteten Hoverkraft-Fahrzeug aufgesucht worden. Dieses wurde freundlicherweise von der kanadischen Coastguard zur Verfügung gestellt. Die übrigen Wattenstationen wurden mit einem Schlauchboot oder zu Fuß besucht und ihre Positionen mit einem Sextanten eingemessen. Die SC-Stationen, die alle im Abwasserkanal lagen, konnten nur mit einem Schlauchboot erreicht werden. Anhand von Sichtzeichen auf der Nordmole ließ sich ihre Position bestimmen.

\section{Hydrographische Messungen}

Der im Wasser gelöste Sauerstoff wurde sowohl nach der Winkler-Methode (Strickland \& Parsons, 1972) als auch membran-polarographisch mit Sauerstoff-Elektroden ermittelt (WTW-Chemograph M 245 und Eo 40 Elektrode).

Die Messungen des Salzgehaltes erfolgte durch Titration des Chlorids mit Silbernitrat (Strickland \& Parsons, 1972) und konduktometrisch mit Feld- und Labor-Salinometer (Beckmann RS 5-3, YSI Modell 33, Autolab $601 \mathrm{Mk}$ III).

Die Ermittlungen der pH-Werte erfolgte mit einem WTW-Feld-pH-Meter, der Temperaturen mit Quecksilber-Thermometern, mit dem WTW-Chemographen und dem Beckmann RS 5-3.

Für die Analysen des gelösten organischen Kohlenstoffs stand ein Beckmann Carbon Analyzer zur Verfügung.

Zur Erfassung des Sestongehaltes und seines Anteils an organischer partikulärer 
Substanz wurden mit einem van Dorn-Wasserschöpfer 1-1-Proben entnommen und formolfixiert; im Labor erfolgte dann die Vakuum-Filtration durch $0,45 \mu$ Whatmann GF/C Glasfaserfilter mit anschließender Trocknung bei $60^{\circ} \mathrm{C}$ über 48 Stunden und die Veraschung bei $550^{\circ} \mathrm{C}$ während 24 Stunden im Muffelofen.

Die Wasserbedeckungszeiten wurden (bei jeder Anwesenheit im Untersuchungsgebiet) durch Beobachtung festgehalten und anhand des Tidenkalenders des Deutschen Hydrographischen Institutes (DHI) in Prozent pro Tag umgerechnet.

Der Abwasserbelastungsfaktor ergab sich für jede Station aus der Abwasserkonzentration und der Zeit, in der diese Konzentration auf das Sediment einwirken kann. Die Abwasserkonzentration war aus dem Salzgehalt des Prielwassers an der betreffenden Station, der Salzkonzentration des eingeleiteten Abwassers und der des aus Seitenprielen zulaufenden Meerwassers zu berechnen.

Für die $\mathrm{O}_{2}$-Dauermessungen registrierte ein WTW-Chemograph (M 245) kontinuierlich die Werte. Die Elektrode (Eo 40) war dazu an einem Ständer dicht über dem Sediment fixiert und durch ein wasserdichtes Kabel mit dem Chemographen am Ufer verbunden.

Bei der Berechnung der Mittelwerte aus den hydrographischen Messungen wurden Extremwerte (z. B. durch starke, kurze Regenfälle) nicht berücksichtigt, weil ihnen für die Charakterisierung der Gesamtsituation nur eine geringe Bedeutung zukommt.

\section{Sedimentanalysen}

Für die Sedimentanalysen wurden Proben von ungestörten Sedimenten mit Plexiglasrohren bis zu einer Tiefe von $5 \mathrm{~cm}$ entnommen und tiefgefroren. Die Analyse der Korngrößen erfolgte durch Naßsiebung mit einem Siebsatz der Wenthworth-Skala. Aus den Gewichtsanteilen der verschiedenen Korngrößen-Fraktionen konnte dann zeichnerisch der Medianwert ermittelt werden. (Zur Problematik der Charkterisierung von Sedimenten durch den Medianwert siehe Trask, 1939; Köster, 1964 und Folk, 1966).

Der Anteil der partikulären organischen Substanzen ergab sich aus dem Glühverlust (vgl. dazu Buchmann \& Longbottom, 1970): Ein Kern der ungestörten oberen Sedimentschicht (1 cm Schichtdicke, etwa 3,5 g Trockengewicht) wurde bei $60^{\circ} \mathrm{C}$ getrocknet und bei $550{ }^{\circ} \mathrm{C}$ vier Stunden geglüht. $\mathrm{Da}$ in den Proben aus dem Untersuchungsgebiet bei Vancouver Holzpartikel enthalten waren, mußten diese Proben 24 Stunden geglüht werden.

Bis zu welcher Tiefe sich im Sediment noch aerobe Prozesse abspielen, kann an der Stärke der braungefärbten Oxydationsschicht eines Schlickkernes erkannt werden. Dazu wurde mit einem graduierten Glasrohr dem ungestörten Sediment ein Kern entnommen und die Höhe von der Oberfläche bis zum Beginn der durch Eisensulfid schwarz gefärbten Zone abgelesen.

\section{Messungen des Chlorophylls im Sediment}

Mit einem Plexiglasrohr von 1,6 cm Durchmesser wurde ein ungestörter Sedimentkern mit einer Oberfläche von $2,45 \mathrm{~cm}^{2}$ und $5 \mathrm{~cm}$ Tiefe im Watt ausgestochen, das Rohr 
oben und unten verkorkt und innerhalb von zwei Stunden nach Zugabe von Magnesiumcarbonat tiefgefroren (je Station zwei Proben). Für die weitere Bearbeitung mußten die Sedimentkerne in gefrorenem Zustand zersägt werden. Die Analyse auf Chlorophyll a und Phaeophytin a der einzelnen 0,5 cm langen Abschnitte erfolgte dann nach der SCORUNESCO-Methode in Anlehnung an Strickland \& Parsons (1972): Das Sediment wurde im Mörser mit $90 \%$ igem Aceton 10 Minuten lang zerrieben, die Pigmente an einem kühlen, dunklen Platz unter häufigem Schütteln vier Stunden lang extrahiert und das Sediment abzentrifugiert. Die Menge der Pigmente ist spektrophotometrisch mit einem PMQ II der Firma Zeiss bzw. einem Carey Modell 15 Spektrophotometer bei einer Wellenlänge von $665 \mathrm{~nm}$ vor und nach Ansäuerung bestimmt worden. Die Analysenwerte werden in Mikrogramm pro Kubikzentimeter angegeben.

\section{Messung der biologischen Aktivität des Sedimentes mit Hilfe der Isotopentechnik}

Die Produktionsmessungen im Sediment lehnten sich an die Methode von Grøntved (1960) an: Ein ungestörter Sedimentkern von 1,6 cm Durchmesser und $1 \mathrm{~cm}$ Tiefe, der vor der Entnahme eine Stunde in situ verdunkelt worden war, wurde mit membrangefiltertem Seewasser durch kräftiges Schütteln in einem Kolben ausgewaschen. Der Vorgang wurde so oft wiederholt, bis keine optisch erkennbare Trübung des Waschwassers mehr sichtbar war und nur Sandkörner und gröbere Partikel zurückblieben. Aus den gesammelten Suspensionen der Auswaschprozedur (max. $200 \mathrm{ml}$ ) wurde nach guter Durchmischung ein 2-ml-Äquivalent mit einer Spritze entnommen und zu gefiltertem Seewasser in $100 \mathrm{ml}$ Flaschen zugegeben. Die Flaschen wurden dann mit $8 \mu \mathrm{Ci} \mathrm{NaH}{ }^{14} \mathrm{CO}_{3}$ zwei Stunden - im Winter drei Stunden - wie bei der Planktonmethode nach Steemann Nielsen (1965) inkubiert. Die Dunkelflaschen waren dazu in Alufolie gewickelt worden. Um eine Erhitzung der Flaschen zu vermeiden, erfolgte ihre Plazierung im Watt so, daß Prielwasser sie ständig umspülte. Zur Vermeidung einer Sedimentation der Bakterien und Algen mußten sie dabei häufig geschüttelt und gewendet werden. Nach Ablauf.der Inkubationszeit wurde die Produktion durch Zugabe von Formol gestoppt, die Flaschen ins Labor gebracht und der gesamte Inhalt auf Membranfilter von 0,3 $\mu$ Porendurchmesser gezogen. Nach Waschen mit verdünnter Salzsäure konnten die Filter getrocknet und bis zum Zählen der Aktivitäten aufbewahrt werden.

Das radioaktive Material sowie die Saug-, Filter- und Trockengeräte waren von der "International Agency for ${ }^{14} \mathrm{C}$-Determination" in Søborg, Dänemark, bezogen worden. Diese Firma nahm auch die Zählungen der Filteraktivitäten vor. Die Korrektur der Ergebnisse zweier Parallelmessungen erfolgte nach Steemann Nielsen (1965). (Angabe der Ergebnisse in $\mathrm{mg}$ Kohlenstoff pro $\mathrm{m}^{2}$ Sedimentoberfläche und Stunde.)

Für die Berechnung der $\mathrm{CO}_{2}$-Dunkelfixierung in bakterielle Produktion (P) geben Sorokin \& Kadota (1972) Umrechnungsfaktoren (f) für unterschiedliche Sedimente an nach der Formel $\mathrm{P}=\mathrm{f} \cdot \mathrm{A}$, wobei $\mathrm{A}$ die $\mathrm{CO}_{2}$-Aufnahme der benthischen Mikroflora bei Dunkelheit (in mg C) angibt. Da im Untersuchungsgebiet auf Sylt der Anteil an organischen Substanzen in und auf dem Sediment recht hoch war, wurde der Faktor 5 benutzt.

Die Bestimmung des Gesamt- $\mathrm{CO}_{2}$-Gehaltes im filtrierten Seewasser erfolgte nach Strickland \& Parsons (1972) und die der Lichtmenge während der Inkubationszeit mit einem Luxmeter der Firma Gossens (Pan Lux). 
In Abweichung zur Grøntved-Methode blieben die an Sandkörnern fest angehefteten Bakterien und Algen unberücksichtigt, da der Sandanteil der Sedimentkerne im Vergleich zur suspendierbaren Fraktion sehr gering war. Außerdem kam es bei diesen Untersuchungen auf graduelle Produktionsunterschiede in Abhängigkeit vom Abwassereinfluß an. Weitere Einzelheiten der Methode siehe Grøntved (1960), Informationen und Gebrauchsanleitung der International Agency, Steemann Nielsen $(1952,1965)$, Cadée \& Hegemann (1974).

\section{Makrobenthos-Untersuchungen}

Um die Ergebnisse der quantitativen Makrobenthos-Analysen aus dem gesamten Untersuchungszeitraum miteinander vergleichen zu können, wurden die Proben fast ausschließlich im Sommerhalbjahr (Juni-September) entnommen. Die Biomassenwerte für das Makrobenthos in Wattengebieten erreichen in diesem Zeitraum ihre Maxima (Hughes, 1970; Longbottom, 1970; Essink, 1972; Hauser, 1973; Beukema, 1974).

An allen Stationen der Sylter Untersuchungsgebiete wurden mit einem Metallzylinder zwei Sedimentsäulen mit einer Oberfläche von je $0,025 \mathrm{~m}^{2}$ (Gesamtfläche $0,05 \mathrm{~m}^{2}$ ) und einer Tiefe von $30 \mathrm{~cm}$ ausgestochen. Im Untersuchungsgebiet bei Vancouver wurde dagegen ein Metallzylinder benutzt, der eine Oberfläche von $0,05 \mathrm{~m}^{2}$ ausstach, so daß nur eine Probe pro Station gewonnen zu werden brauchte. Die Sedimentkerne wurden im Labor durch ein Sieb mit einer Maschenweite von 0,8 mm gespült. Diese Weite erwies sich als annehmbarer Kompromiß, um einerseits den Siebrest so gering zu halten, daß das Aussuchen der Tiere auf ein arbeitsmäßig vertretbares $\mathrm{Maß}$ reduziert bleibt, andererseits Organismen von $1 \mathrm{~mm}$ Größe noch weitgehend zurückgehalten werden.

Die Identifizierung der Fauna aus den Sylter Watten erfolgte mit nachstehenden Bestimmungsschlüsseln und Artenbeschreibungen: Polychaeta: Hartmann-Schröder (1971), Friedrich (1938), Fauvel (1923, 1928); Crustacea: Schellenberg (1942), Crawford (1937); Molluska: Tebble (1966), Ziegelmeier (1957). Die Hydrobien wurden wegen ihrer gewaltigen Individuenzahlen nicht artlich differenziert. Wenn auch in Stichproben nur $H y d r o b i a$ ulvae gefunden wurde, kann nicht ausgeschlossen werden, daß auch $H$. neglecta und $H$. ventrosa dort vorkommen (Muus, 1967).

Die Fauna aus dem pazifischen Wattengebiet bei Vancouver wurde mit folgender Literatur artlich determiniert: Polychaeta mit veröffentlichten und teilweise unpublizierten Bestimmungsschlüsseln von Banse \& Hobson (1974) sowie Erläuterungen von Hobson (Provincial Museum, Victoria, British Columbia), ferner mit Schlüsseln von Berkeley \& Berkeley (1952), Uschakov (1955) und Day (1967). Amphipoden: mit den Beschreibungen von Barnard (1959, 1969), Shoemaker (1934 a, b; 1941, 1949), Crawford (1937) und Otte (1975). Mysidacea und Decapoda wurden von McDaniel, Pacific Environment Institute, bestimmt und die Mollusca mit Hilfe der Beschreibungen von Quayle (1960) und Rice (1972).

Die Oligochaeten und Nemertinen sind nicht artlich differenziert worden. Viele Oligochaeten-Arten können nur lebend sicher bestimmt werden. Deshalb sind sie auch nur, ebenso wie die Nemertinen und die Hydrobien aus den Sylter Watten, summarisch erfaßt worden. 


\title{
ERGEBNISSE
}

Vorbehandlung und Qualität der eingeleiteten Abwässer

\author{
Sylt
}

Die Kläranlagen der Inselgemeinden reinigen nur häusliches Abwasser. Die anfallenden Mengen sind großen saisonalen Schwankungen unterworfen. Wegen der Unterschiede in der Abwasserbehandlung und der daraus resultierenden qualitativen Verschiedenheit der Vorflutereinleitungen werden die drei untersuchten Anlagen getrennt betrachtet.

$\mathrm{K}$ a m p e n. Während der Voruntersuchungen 1970 wurde das Abwasser von Kampen noch ganzjährig mittels eines Schlackentropfkörpers biologisch gereinigt. Dieses erwies sich aber in den Sommermonaten wegen der starken Zunahme des Tourismus als nicht ausreichend (etwa 16000 Kurgäste pro Jahr bei 900 Einwohnern). Daher wurde die Anlage im darauffolgenden Jahr erweitert und das Abwasser während des Sommers nach dem Belebtschlammverfahren geklärt. Der Vorteil dieser Methode zeigt sich an den Analysendaten aus dem Ablauf der Kläranlage (Tab. 1): niedrige BSB $_{5}$-Werte und relativ hohe Sauerstoffwerte (besonders in den Sommermonaten) sowie ein geringer Gehalt an organischen Sinkstoffen weisen auf eine gute Abbauleistung hin. Der $\mathrm{pH}$-Wert und der niedrige Phosphatgehalt waren an allen Untersuchungstagen sehr konstant. Unregelmäßigkeiten zeigen sich nur bei der Nitrifikation $\left(\mathrm{NH}_{4}-\mathrm{N}-, \mathrm{NO}_{2}-\mathrm{N}-, \mathrm{NO}_{3}-\mathrm{N}\right.$-Werte), die erfahrungsgemäß bei der kurzen Verweilzeit des Abwassers im Klärwerk unvollständig bleiben muß (Downing \& Wheatland, 1962; Johnson \& Schroepfer, 1964). Diese Werte hängen mehr vom zufließenden, ungereinigten $A b w a s s e r$ ab als vom Klärprozeß selbst.

Bei der Beurteilung der in Tabelle 1 angegebenen Analysenwerte muß allerdings berücksichtigt werden, daß das Abwasser vom Klärwerk bis zum Abfluß ins Watt einen längeren Graben durchfließt, die Abwasserwerte sich folglich noch ändern können. So wurde trotz des noch ausreichend vorhandenen $\mathrm{O}_{2}$-Gehaltes im Ablauf der Anlage bei der Ankunft des Abwassers im Watt häufig kein Sauerstoff mehr nachgewiesen. Man kann also davon ausgehen, daß das ins Watt einfließende Abwasser zeitweise sauerstofffrei ist.

Wegen der geringen Abwassermenge im Winterhalbjahr wird ab Herbst der Tropfkörper wieder benutzt; die Belebtschlammanlage wäre nun überdimensioniert. Dieses Verfahren - zwei verschiedene Anlagen zu benutzen - hat jedoch einen entscheidenden Nachteil: erfahrungsgemäß benötigt eine biologische Kläranlage einige Zeit, bis sie ihre volle Klärleistung erreicht. In dieser Zeit wird unvollständig gereinigtes Abwasser in den Vorfluter - hier also ins Wattengebiet - eingeleitet.

$\mathrm{L}$ i s t. Im Gegensatz zu Kampen wird von dieser Gemeinde das Abwasser der rund 4000 Einwohner und 3000 Kurgästen (Tagesdurchschnitt der Sommermonate) lediglich mechanisch gereinigt. Das abfließende Wasser ist in der Regel $\mathrm{O}_{2}$-frei; eine Teilnitrifikation ist daher nicht möglich (s. hohe $\mathrm{NH}_{4}-\mathrm{N}$-Werte, kein Nitrit und Nitrat: Tab. 1). Die hohen $\mathrm{BSB}_{5}-$ Werte (bis zu $475 \mathrm{mg} / \mathrm{l}$ ) bei verhältnismäßig geringem Anteil an organischen partikulären Substanzen deuten zwar auf eine wirksame mechanische Reinigungsleistung hin, zeigen jedoch, daß ein Abbau der organischen gelösten Stoffe nicht stattfindet.

Das anaerobe Abwasser mit seinen eventuell toxischen Inhaltsstoffen wirkt sich nicht direkt auf die Wattflächen aus, da es bei auflaufender Tide mit dem Meerwasser vermischt 
Tabelle 1

Charakteristik der eingeleiteten Abwässer. Analysenwerte vom Kläranlagenablauf in Kampen, List und Braderup (Sylt). (Werte aus dem städrischen Laboratorium Kiel, Dr. Heincke; Angaben in mg/l)

\begin{tabular}{|c|c|c|c|c|c|c|c|c|}
\hline $\begin{array}{l}\text { Datum der } \\
\text { Probennahmen }\end{array}$ & $\begin{array}{l}16.2 . \\
1971\end{array}$ & $\begin{array}{l}5.8 . \\
1971\end{array}$ & $\begin{array}{l}26.4 . \\
1972\end{array}$ & $\begin{array}{l}3.8 . \\
1972\end{array}$ & $\begin{array}{c}28.3 . \\
1973\end{array}$ & $\begin{array}{l}26.6 . \\
1973\end{array}$ & $\begin{array}{l}30.8 . \\
1973\end{array}$ & $\begin{array}{l}6.11 . \\
1973\end{array}$ \\
\hline \multicolumn{9}{|l|}{$\mathrm{K}$ a mpen } \\
\hline \multirow{3}{*}{$\begin{array}{l}\mathrm{pH} \\
\text { org. Sink- } \\
\text { stoffe } \\
\mathrm{BSB}_{5} \\
\mathrm{O}_{2} \\
\mathrm{PO}_{4}-\mathrm{P} \\
\mathrm{NH}_{4}-\mathrm{N} \\
\mathrm{NO}_{2}-\mathrm{N} \\
\mathrm{NO}_{3}-\mathrm{N}\end{array}$} & 7,45 & 7,6 & 7,7 & - & 7,19 & 7,97 & 7,73 & 7,21 \\
\hline & 15,4 & 6,6 & 3,7 & - & 29,6 & 12,8 & 14,2 & 12,0 \\
\hline & $\begin{array}{c}83 \\
5,9 \\
44 \\
41,2 \\
3,2 \\
6,6\end{array}$ & $\begin{array}{c}32 \\
2,7 \\
45 \\
48,6 \\
0,0 \\
0,0\end{array}$ & $\begin{array}{c}65 \\
5,2 \\
63 \\
49,7 \\
1,1 \\
0,0\end{array}$ & $\begin{array}{l}- \\
- \\
- \\
- \\
- \\
-\end{array}$ & $\begin{array}{c}22 \\
9,6 \\
58 \\
33,1 \\
0,4 \\
23,0\end{array}$ & $\begin{array}{c}3 \\
4,3 \\
54 \\
45,7 \\
1,4 \\
3,4\end{array}$ & $\begin{array}{c}6 \\
3,6 \\
48 \\
40,3 \\
2,0 \\
0,0\end{array}$ & $\begin{array}{l}23 \\
10,1 \\
59 \\
23,4 \\
0,0 \\
6,4\end{array}$ \\
\hline \multicolumn{9}{|l|}{ Lis $t$} \\
\hline \multirow{8}{*}{$\begin{array}{l}\mathrm{pH} \\
\text { org. Sink- } \\
\text { stoffe } \\
\mathrm{BSB}_{5} \\
\mathrm{O}_{2} \\
\mathrm{PO}_{4}-\mathrm{P} \\
\mathrm{NH}_{4}-\mathrm{N} \\
\mathrm{NO}_{2}-\mathrm{N} \\
\mathrm{NO}_{3}-\mathrm{N}\end{array}$} & - & - & 7,24 & 7,7 & 6,86 & - & 7,64 & - \\
\hline & - & - & 40,6 & 29,8 & 38,0 & - & 19,6 & - \\
\hline & - & - & 167 & 475 & 271 & - & 250 & - \\
\hline & - & - & 0,0 & 0,0 & 3,2 & - & 0,0 & - \\
\hline & - & - & 69 & 76 & 43 & - & 43 & - \\
\hline & - & - & 69,1 & 83,9 & 51,8 & - & 74,2 & - \\
\hline & - & - & 0,0 & 0,0 & 0,0 & - & 0,0 & - \\
\hline & - & - & 3,6 & 0,0 & 0,0 & - & 0,0 & - \\
\hline \multicolumn{9}{|l|}{ Braderup } \\
\hline \multirow{2}{*}{$\begin{array}{l}\text { pH } \\
\text { org. Sink- } \\
\text { stoffe }\end{array}$} & - & - & 7,8 & 7,57 & 7,17 & - & 7,86 & - \\
\hline & - & - & 21,8 & 83,2 & 56,4 & - & 30,4 & - \\
\hline $\mathrm{BSB}_{5}$ & - & - & 14 & 294 & 27 & - & 52 & - \\
\hline $\mathrm{O}_{2}$ & - & - & 7,9 & 0,0 & 10,6 & - & 2,3 & - \\
\hline $\mathrm{PO}_{4}^{-}-\mathrm{P}$ & - & - & 73 & 75 & 69 & - & 39 & - \\
\hline $\mathrm{NH}_{4}-\mathrm{N}$ & - & - & 22,3 & 98,5 & 21,6 & - & 59,8 & - \\
\hline $\mathrm{NO}_{2}-\mathrm{N}$ & - & - & 1,9 & 2,4 & 0,5 & - & 0,8 & - \\
\hline $\mathrm{NO}_{3}-\mathrm{N}$ & - & - & 31,1 & 0,0 & 0,0 & - & 0,0 & - \\
\hline
\end{tabular}

wird und in die oben beschriebene Lagune abfließt. Während der Niedrigwasserphase läuft dieses Gemisch zusammen mit dem Kläranlagenabfluß in einen seichten Priel durch die kleine Wattenbucht (s. Abb. 1). Dabei nimmt es geringfügig Sauerstoff auf. Erst wenn das aufgestaute Wasser aus der Lagune abgelaufen ist, fließt in der letzten Niedrigwasserphase konzentriertes, unverdünntes und damit $\mathrm{O}_{2}$-freies Abwasser direkt aus der Kläranlage über das Wattensediment.

$\mathrm{B} \mathrm{r}$ a d e r u p. Die Kläranlage dieses Ortes besitzt eine mechanische und eine biologische Reinigungsstufe (geschlossener Schlackentropfkörper). Sie ist im Winterhalbjahr für die etwa 2000 Einwohner ausreichend, im Sommer aber völlig überlastet (etwa 28000 Kurgäste pro Saison). Der geringe bis fehlende Sauerstoff, der hohe BSB $_{5}$-Wert (bis zu 294 $\mathrm{mg} / \mathrm{l}$ ) und der große Anteil an organischer partikulärer Substanz im Sommerhalbjahr (max. $83,2 \mathrm{mg} / \mathrm{l}$ ) beweisen dies (Tab. 1). Die Phosphat-Werte liegen auch außerhalb der Saison 
relativ hoch, während die Nitrifikation - wie auch in Kampen - nur zögernd einsetzt. Das Abwasser wird vom Klärwerk durch einen unterirdischen Düker direkt ins Watt geleitet. Am Auslauf war es stets sauerstofffrei, auch wenn am Kläranlagenabfluß ein Restgehalt von $\mathrm{O}_{2}$ festgestellt werden konnte.

\section{Vancouver}

Ein Mischabwasser kommunalen und industriellen Ursprungs wird in das Wattengebiet des Fraser River bei Vancouver eingeleitet. Es wird lediglich mechanisch gereinigt und der Ablauf gechlort (etwa $1 \mathrm{ppm} \mathrm{Cl}{ }^{-}$). An das Klärwerk angeschlossen sind rund 400000 Einwohner und Kleinbetriebe, jedoch keine Großindustrie. Die täglich im Untersuchungszeitraum (Mai 1974-Januar 1975) eingeleitete Abwassermenge betrug durchschnittlich 273 $\times 10^{3} \mathrm{~m}^{3}$ mit einem BSB $_{5}$-Wert von 65-130 mg/l. Der Sauerstoffgehalt an der Einleitungsstelle schwankte von Juni bis Oktober zwischen 1,9 und $5,2 \mathrm{mg} / \mathrm{l}$, der $\mathrm{pH}$-Wert lag zwischen 6,8 und 7,0. Der Sestongehalt im Abwasser betrug 42-62 mg/l, wovon durchschnittlich $88 \%$ organischer Natur war.

\section{Beeinflussung der chemisch-physikalischen Umweltfaktoren im "Vorfluter Watt" durch das Abwasser}

Eine Abwassereinleitung in Wattengebiete beeinflußt zwei voneinander abgrenzbare Biotope: (1) Die Priele und Kanäle, in denen das Abwasser lediglich bei Niedrigwasser abfließt, und (2) die Wattflächen, auf denen es sich während der Uberflutungszeiten ausbreitet. Dieser zeitlich und räumlich unterschiedliche Einfluß auf beide Lebensräume ließ sich durch die hydrochemischen Messungen deutlich nachweisen. Es war daher sinnvoll, die Beeinflussung der chemisch-physikalischen Faktoren bei unterschiedlichen Tidenphasen örtlich getrennt nach Priel (bzw. Kanal) und Wattflächen zu analysieren.

\section{Einfluß des Abwassers auf den Sauerstoffgehalt des Wattes}

Die Erhaltung eines ausreichenden Sauerstoffangebotes für die Lebensgemeinschaften im Vorfluter ist bei kommunalen Abwassereinleitungen problematisch. Sind der Sauerstoffeintrag in den Vorfluter und die für die Dekomposition der Abwasserinhaltsstoffe notwendige Sauerstoffmenge bekannt, so kann abgeschätzt werden, ob eine kontinuierlich einzuleitende Abwassermenge zu Sauerstoffdefiziten bzw. zu anaeroben Bedingungen führt. Eine solche - rein theoretische - Berechnung ist für das Untersuchungsgebiet Vancouver überschlagsmäßig durchgeführt worden: Für die Dekomposition der täglichen Abwassermenge von durchschnittlich $273 \times 10^{3} \mathrm{~m}^{3}$ mit einem BSB $_{5}$-Wert von $65-130 \mathrm{mg} / \mathrm{l}$ würden etwa $25 \times 10^{3} \mathrm{~kg} \mathrm{O}_{2}$ verbraucht werden. Ausgehend von einer im Einflußbereich der Abwassereinleitung liegenden Wattfläche von $8 \mathrm{~km}^{2}$ kann unter Berücksichtigung der Bodentopographie ein mittleres Wasservolumen von $12 \times 10^{6} \mathrm{~m}^{3}$ angenommen werden. Somit wird bei einmaligem Austausch dieses Wasserkörpers eine Sauerstoffmenge von ca. 
$108 \times 10^{3} \mathrm{~kg} \mathrm{O}_{2}$ für den Abbau verfügbar gemacht, wenn man einen mittleren Sauerstoffgehalt des einströmenden Meerwassers von $9 \mathrm{mg} / \mathrm{l}$ annimmt. Diese Zahlen verdeutlichen, daß theoretisch das Sauerstoffangebot im Wasser für den biologischen Abbau der Abwasserfracht ausreichen würde, wenn das Abwasser gleichmäßig über das Watt verteilt werden könnte. Darüber hinaus steht für den Abbau der sedimentierten Partikel auf der Sedimentoberfläche bei Niedrigwasser der Luftsauerstoff zur Verfügung. Ferner muß berücksichtigt werden, daß innerhalb von $24 \mathrm{~h}$ zwei Tidenzyklen ablaufen, sich also die obige Kalkulation des Sauerstoffangebotes für den Abbau noch günstiger darstellt.

In der Praxis wird Abwasser jedoch punktförmig ins Watt eingeleitet. Eine deutliche Beeinträchtigung des Sauerstoffhaushaltes findet man daher unmittelbar an den $\mathrm{E}$ i n l e i t u n g s t e 1 l e n. Wegen längerer Zuleitungswege von den Kläranlagen weist das Abwasser beim Eintritt ins Watt meist nur noch einen geringen oder gar keinen Gehalt an gelöstem Sauerstoff auf; der noch am Kläranlagenablauf nachzuweisende $\mathrm{O}_{2}$-Gehalt (Tab. 1) wird durch hohe BSB $_{5}$-Belastungen während der Zuleitung weggezehrt. So zeigten Messungen in Braderup ebenso wie in List und Kampen (nur Beobachtungsjahr 1970) an der Einleitungsstelle zu keiner Zeit Spuren von Sauerstoff. Erst nachdem die biologische

Tabelle 2

Einige Umweltfaktoren im Abwasserpriel von Kampen (Sylt) bei Niedrigwasser. Daten aus verschiedenen Beobachtungsjahren (jeweils zwischen Juni und September). $\mathrm{n}=$ Anzahl der Beobachtungswerte; $\overline{\mathrm{x}}=$ Mittelwert; $s=$ Standardabweichung; DOC $=$ gelöster organischer Kohlenstoff. Berechnung der Abwasserkonzentration und des Abwasser-Belastungsfaktors vgl. p. 95. Die Werte für die Uberflutungszeiten können in Abhängigkeit vom Wind um 20-30\% schwanken

\begin{tabular}{|c|c|c|c|c|c|c|c|c|c|c|c|}
\hline \multirow[b]{2}{*}{ Faktoren } & & \multicolumn{10}{|c|}{$\begin{array}{l}\text { Probenstationen } \\
\text { und ihre Entfernungen von der Einleitungsstelle (m) }\end{array}$} \\
\hline & & $\mathbf{n}$ & $\begin{array}{c}\mathrm{K} O \\
0\end{array}$ & $\begin{array}{c}\mathrm{K} 1 \\
50\end{array}$ & $\begin{array}{l}\mathrm{K} 2 \\
100\end{array}$ & $\begin{array}{l}K 3 \\
150\end{array}$ & $\begin{array}{l}\mathrm{K} 4 \\
200\end{array}$ & $\begin{array}{l}\text { K } 6 \\
300\end{array}$ & $\begin{array}{l}K 8 \\
400\end{array}$ & $\begin{array}{c}\mathrm{K} 10 \\
500\end{array}$ & $\underset{600}{K} 12$ \\
\hline $\begin{array}{l}\mathrm{O}_{2}(\mathrm{mg} / \mathrm{l}) \\
1970\end{array}$ & $\begin{array}{l}\overline{\mathbf{x}} \\
\mathrm{s}\end{array}$ & 9 & $\begin{array}{l}0,0 \\
0,0\end{array}$ & $\begin{array}{l}0,8 \\
0,48\end{array}$ & $\begin{array}{l}2,8 \\
1,33\end{array}$ & $\begin{array}{l}3,5 \% \\
0,77\end{array}$ & $\begin{array}{l}6,8 \\
0,25\end{array}$ & $\begin{array}{l}8,2 \\
0,69\end{array}$ & $\begin{array}{l}8,6 \\
0,86\end{array}$ & $\begin{array}{l}9,2 \\
0,46\end{array}$ & $\begin{array}{l}8,6 \\
0,28\end{array}$ \\
\hline $\begin{array}{l}\mathrm{O}_{2}(\mathrm{mg} / \mathrm{l}) \\
1971-73\end{array}$ & $\begin{array}{l}\widetilde{\mathbf{x}} \\
\mathrm{s}\end{array}$ & 5 & $\begin{array}{l}0,5 \\
0,6\end{array}$ & $\begin{array}{l}2,7 \\
1,23\end{array}$ & $\begin{array}{l}3,4 \\
0,96\end{array}$ & $\begin{array}{l}4,0 \\
0,81\end{array}$ & $\begin{array}{l}6,9 \\
0,3\end{array}$ & $\begin{array}{l}8,1 \\
0,52\end{array}$ & $\begin{array}{l}8,9 \\
0,72\end{array}$ & $\begin{array}{l}8,9 \\
0,33\end{array}$ & $\begin{array}{l}8,4 \\
0,15\end{array}$ \\
\hline $\begin{array}{l}S \% \\
1970-73\end{array}$ & $\begin{array}{l}\overline{\mathrm{x}} \\
\mathrm{s}\end{array}$ & 7 & $\begin{array}{l}2,43 \\
0,31\end{array}$ & $\begin{array}{l}2,94 \\
0,74\end{array}$ & $\begin{array}{l}4,07 \\
1,88\end{array}$ & $\begin{array}{l}6,45 \\
2,54\end{array}$ & $\begin{array}{r}15,14 \\
9,14\end{array}$ & $\begin{array}{r}22,87 \\
7,88\end{array}$ & $\begin{array}{r}25,4 \\
5,5\end{array}$ & $\begin{array}{c}26,05 \\
5,7\end{array}$ & $\begin{array}{r}26,21 \\
5,37\end{array}$ \\
\hline $\begin{array}{l}\mathrm{pH} \\
1970-73\end{array}$ & $\begin{array}{l}\overline{\mathrm{x}} \\
\mathrm{s}\end{array}$ & 6 & $\begin{array}{l}7,45 \\
0,16\end{array}$ & $\begin{array}{l}7,75 \\
0,05\end{array}$ & $\begin{array}{l}8,06 \\
0,09\end{array}$ & $\begin{array}{l}8,3 \\
0,09\end{array}$ & $\begin{array}{l}8,79 \\
0,12\end{array}$ & $\begin{array}{l}8,78 \\
0,03\end{array}$ & $\begin{array}{l}8,8 \\
0,05\end{array}$ & $\begin{array}{l}8,81 \\
0,02\end{array}$ & $\begin{array}{l}8,9 \\
0,07\end{array}$ \\
\hline $\begin{array}{l}\mathrm{DOC}(\mathrm{mg} / \mathrm{l}) \\
1970\end{array}$ & $\overline{\mathbf{x}}$ & 2 & 50,9 & 52,7 & 39,5 & - & 34,8 & 14,5 & 18,5 & 16,7 & 13,8 \\
\hline $\begin{array}{l}\text { Uberflutungs- } \\
\text { zeit }(\% / \mathrm{d})\end{array}$ & & & 18,1 & 41,7 & 43,3 & 45,0 & 48,6 & 56,8 & 58,6 & 64,1 & 70,1 \\
\hline $\begin{array}{l}\text { Abwasser- } \\
\text { konzentration }(\%)\end{array}$ & & & 100 & 97,6 & 93,9 & 84,2 & 50,2 & 20,0 & 10,2 & 7,6 & 7,0 \\
\hline $\begin{array}{l}\text { Abwasserbela- } \\
\text { stungsfaktor }\end{array}$ & & & 0,82 & 0,57 & 0,53 & 0,46 & 0,26 & 0,08 & 0,04 & 0,03 & 0,02 \\
\hline$* \mathrm{n}=5$ & & & & & & & & & & & \\
\hline
\end{tabular}


Abwasserreinigung im Klärwerk Kampen (ab 1972) verbessert wurde, konnte hier ein durchschnittlicher Sauerstoffgehalt von $0,5 \mathrm{mg} / \mathrm{l}$ im Abwasser nachgewiesen werden (Tab. 2).

Der geringe oder fehlende $\mathrm{O}_{2}$-Gehalt im eingeleiteten Abwasser wirkt sich nur bei Niedrigwasser auf das Sediment an der Einleitungsstelle aus. Wie Dauermessungen bei Kampen und List zeigen, stieg der $\mathrm{O}_{2}$-Gehalt am Boden mit auflaufendem Wasser sofort steil an und fiel erst wieder auf Null zurück, wenn das Meerwasser ablief und konzentriertes Abwasser über das Sediment floß (Abb. 3 und 4). Der Grund für den steilen Anstieg der Sauerstoffwerte ist darin zu sehen, daß das auflaufende Meerwasser das Abwasser zunächst in den Zuleitungsgraben zurückdrängte und dann wegen seiner geringeren Dichte an der Wasseroberfläche ablief.

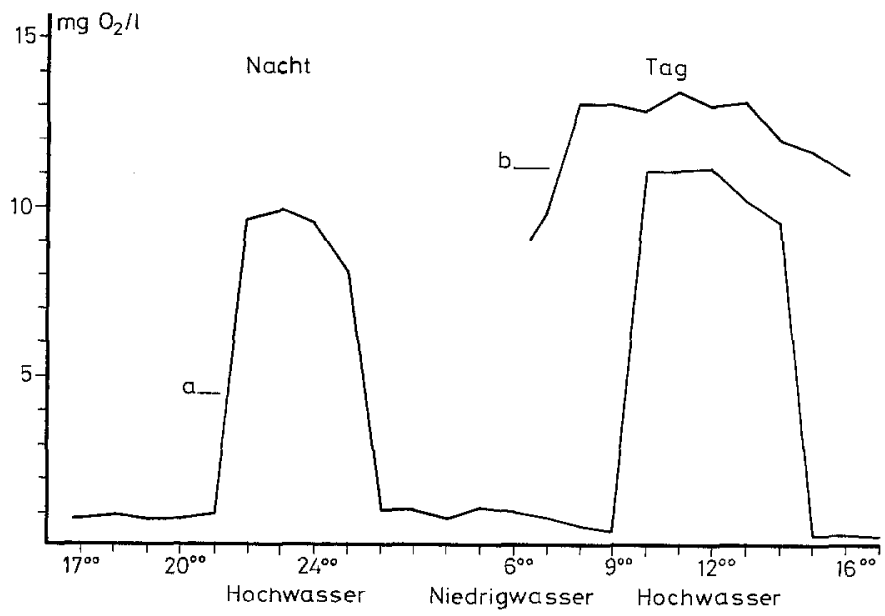

Abb. 3: Untersuchungsgebiet Kampen/Sylt. Abhängigkeit des Sauerstoffgehaltes von den Gezeiten. Kontinuierliche Aufzeichnung der Meßwerte in unmittelbarer Nähe der Einleitungsstelle (19.-20. Okt. 1972): a) im Abwasserpriel, b) auf der angrenzenden trockenfallenden Wattfläche (nach Otte, 1977; verändert)

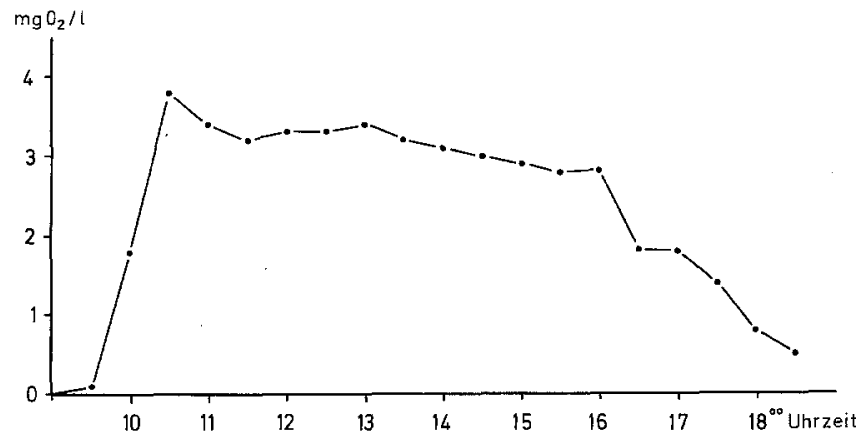

Abb. 4: Untersuchungsgebiet List/Sylt. Sauerstoffgehalt im tidenüberströmten Kläranlagenablauf (22. Juni 1972) 


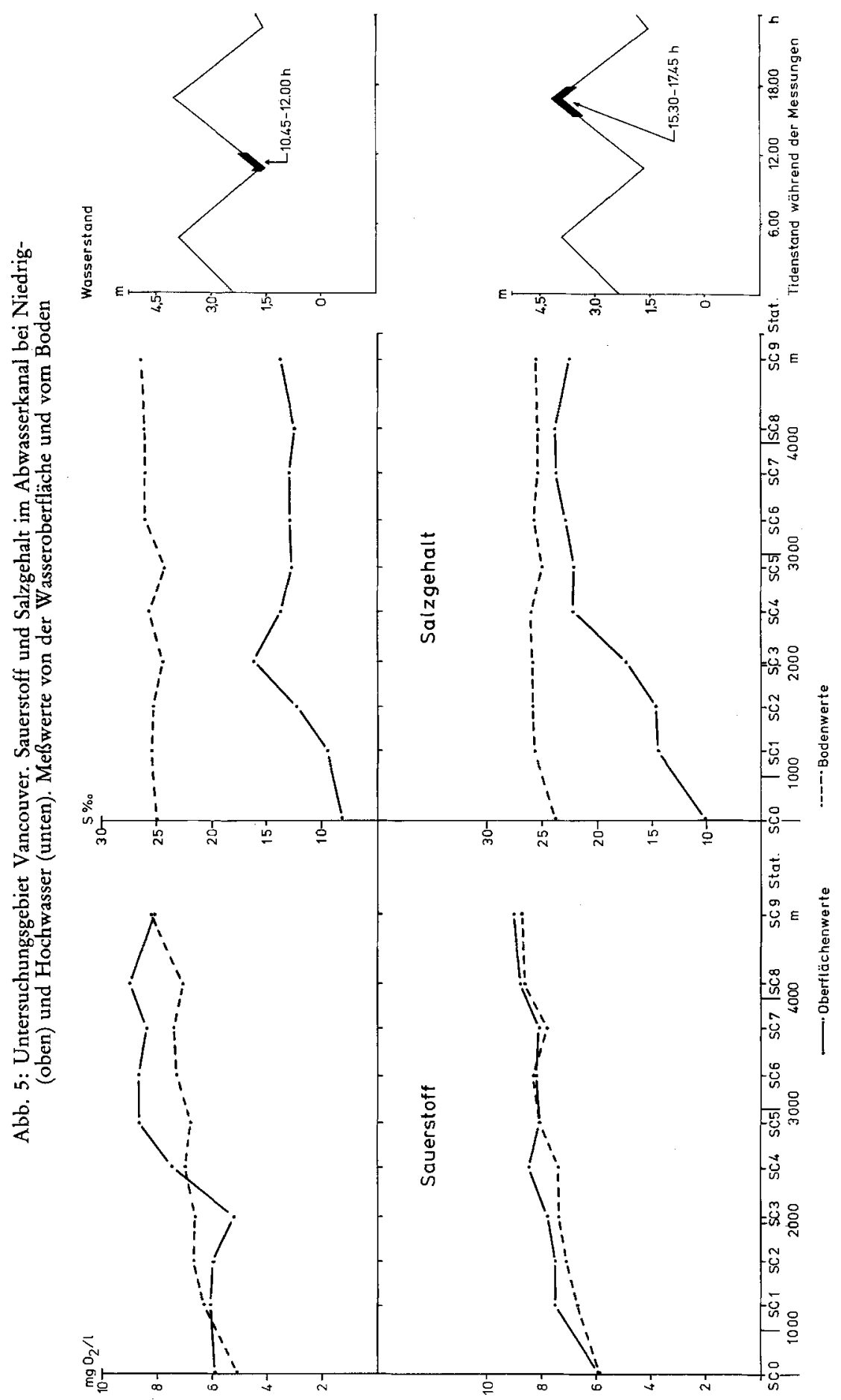


Wie das Beispiel der Abwassereinleitung bei Vancouver zeigt, läßt sich allerdings ein hohes Sauerstoffdefizit an der Einleitungsstelle selbst bei Niedrigwasser vermeiden (Abb. 5): Das Abwasser fließt vom Klärwerk über Betonstufen kaskadenartig in den Kanal, wobei es mit Sauerstoff angereichert wird. Dennoch kam es hin und wieder zu kritischen Sauerstoffverhältnissen, wie einzelne Messungen belegen: bei ruhigen Wetterlagen ist am Boden mehrfach nur ein $\mathrm{O}_{2}$-Gehalt von $0,6 \mathrm{mg} / \mathrm{l}$ gefunden worden.

Abgesehen von der unmittelbaren Einleitungsstelle wird der Sauerstoffhaushalt ebenfalls im oberen Teil des $\mathrm{A} \mathrm{b}$ w a s s e r p ri e l s beeinträchtigt. Bei Niedrigwasser fließt das Abwasser hier (hoch) konzentriert über das Sediment. Bei ausreichender Verdünnung mit Meerwasser aus Seitenprielen macht sich die $\mathrm{O}_{2}$-Zehrung des Abwassers dann im weiteren Prielverlauf kaum noch bemerkbar. Regelmäßige Messungen des gelösten Sauerstoffs im Abwasserpriel bei Kampen zeigten, daß erst nach $200 \mathrm{~m}$ Fließstrecke (bei Station $\mathrm{K} 4$ ) der $\mathrm{O}_{2}$-Wert auf über $4 \mathrm{mg} / \mathrm{l}$ anstieg (Tab. 2). Hier wurde das Abwasser durch sauerstoffreiches Seewasser aus einem einmündenden Priel um etwa $50 \%$ verdünnt. Erst nach $300 \mathrm{~m}$ (Station K 6) wurden Werte über $8 \mathrm{mg} / 1$ erreicht - Werte, wie sie auch im unbelasteten Meerwasser zu finden waren.

Im $\mathrm{Ab}$ w a s s e $\mathrm{rkan}$ a l bei Vancouver war im oberen Abschnitt ebenfalls eine $\mathrm{O}_{2}$-Erniedrigung festzustellen. $\mathrm{Da}$ das einfließende Abwasser aber relativ sauerstoffreich war (s. o.), es sich im rund $2 \mathrm{~m}$ tiefen Kanal teilweise schon an der Einleitungsstelle mit Seewasser vermischte und dann an der Oberfläche abfloß, blieb hier der Sauerstoffgehalt am Boden sowohl bei Hoch- als auch bei Niedrigwasser in der Regel über $3 \mathrm{mg} / \mathrm{l}$.

Auf den Wattflächen, die nicht direkt neben der Einleitungsstelle liegen, kommt es im allgemeinen während der Niedrigwasserzeit nicht zu Sauerstoffdefiziten an der Sedimentoberfläche, da der hohe Sauerstoffanteil der Luft Zutritt zum Sediment hat. Nur auf den Wattstreifen neben den Abwasserprielen wurde in einigen Ausnahmefällen die Sedimentoberfläche an Stellen, die bei Niedrigwasser von einem dünnen Wasser-Abwasserfilm bedeckt waren, nicht oxydiert, so daß das Sediment dort schwarz blieb.

Während der Uberflutung der Wattflächen ist ebenfalls nur selten und kurzfristig ein Sauerstoffdefizit festzustellen, sofern es sich um die Einleitung geringer $\mathrm{A}$ b w a s s e r m e n g e h handelt. Der Verlauf der Sauerstoffkurve an festen Meßpunkten entspricht jedoch dem typischen Bild, wie es bereits oben für den Abwasserpriel selbst beschrieben wurde. Wie die Beispiele für Kampen (Abb. 3) und List (Abb. 4) zeigen, war der $\mathrm{O}_{2}$-Gehalt des auflaufenden Seewassers anfangs noch erniedrigt, da das Abwasser, das noch im Priel ablief, vom einfließenden Seewasser aufgestaut und über die Wattflächen geschoben wurde. Durch das nachströmende frische Meerwasser erhöhten sich die Sauerstoffwerte schnell und fielen erst wieder kurz vor dem Trockenfallen des Sedimentes ab, da vorübergehend das Abwasser hinter dem zurückweichenden Meerwasser nachlief.

Größere Abwassermengen können gelegentlich auch während der gesamten Uberflutungsphase auf den Wattflächen lokal kritische Sauerstoffverhältnisse verursachen. $\mathrm{O}_{2}$-Messungen bei ruhigem Wetter im Untersuchungsgebiet von Vancouver (Wassertiefe 0,5 bis 1,5 m) zeigten deutlich, wie sich das auflaufende, frische Seewasser in einem Keil gegen die Einleitungsstelle heranschob und dabei das Abwasser in den oberen Kanalabschnitt sowie gegen die Küste und in die kleine Bucht unterhalb der Einleitungsstelle zurückdrängte (Abb. 6). Allerdings wurden die niedrigsten Sauerstoffwerte nicht im unmittelbaren Bereich um die Einleitungsstelle gemessen, sondern in der kleinen Bucht 


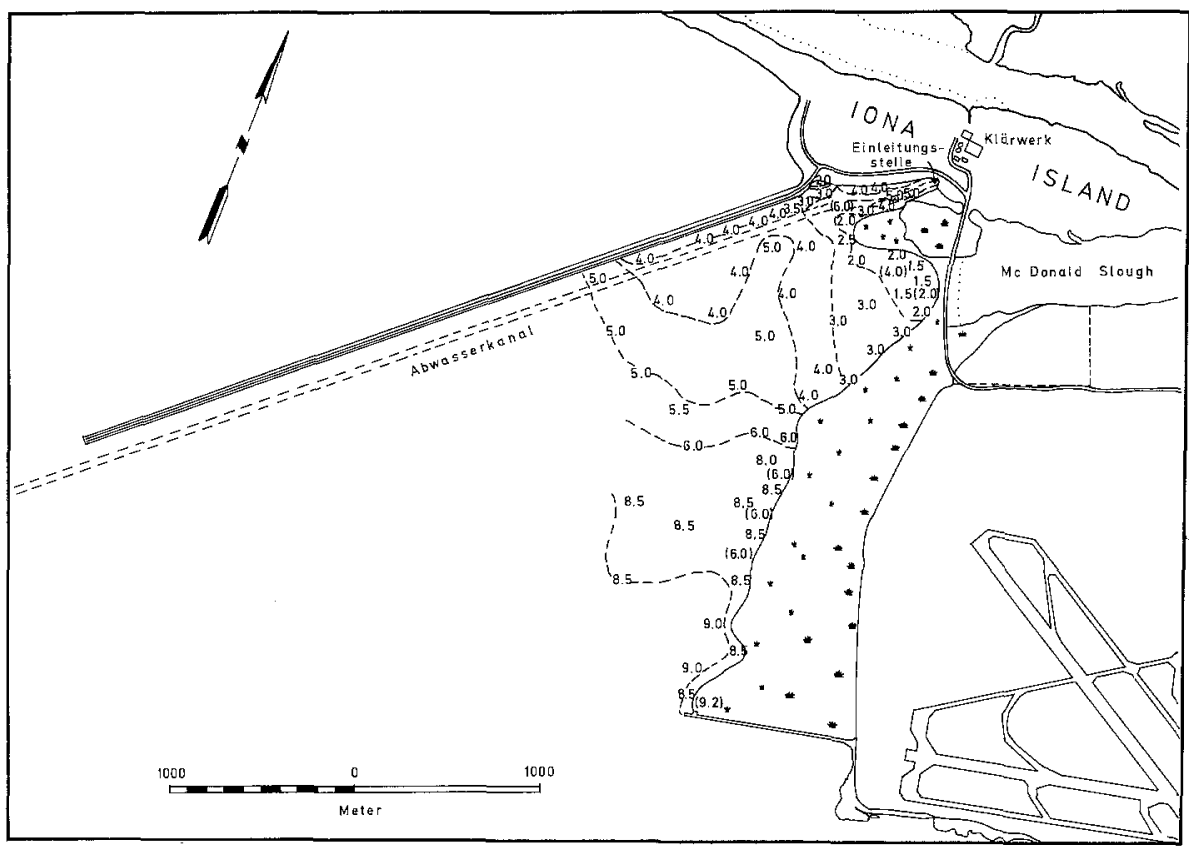

Abb. 6: Untersuchungsgebiet Vancouver. Sauerstoffmessungen bei Hochwasser. Die unterbrochenen Linien grenzen Zonen gleichen Sauerstoffgehaltes voneinander ab. Meßwerte aus der jeweils mittleren Wassertiefe. In Klammern: Sauerstoffgehalt direkt über dem Sediment

sïdlich davon. Hier sank der aktuelle Sauerstoffgehalt im Wasser bis auf 1,5 mg/l ab. Mit zunehmender Entfernung vom Verunreinigungszentrum stieg der Sauerstoffgehalt seewärts auf solche Werte an, wie sie unbelastetem Seewasser entsprechen. Für die relativ guten $\mathrm{O}_{2}$-Werte an der Einleitungsstelle sind die obengenannten Gründe verantwortlich.

Einzelne Messungen direkt an der Sedimentoberfläche ergaben in stark belasteten Zonen regelmäßig höhere $\mathrm{O}_{2}$-Werte als im darüberstehenden Wasser (Abb. 6: Werte in Klammern). Dies zeigt wieder, daß das Meerwasser bei Flut auf dem Boden einfließt und das Abwasser an die Oberfläche verdrängt, wie durch Salzgehaltmessungen bestätigt werden konnte (s. a. Tabata et al., 1971). Dadurch wird das Gebiet mit hohen Sauerstoffdefiziten stark eingeschränkt. In Bereichen geringerer Belastungen ( $\mathrm{ab} 8 \mathrm{mg} / \mathrm{l}$ aktuellem $\mathrm{O}_{2}$ Gehalt) liegen dagegen die Sauerstoffwerte in Sedimentnähe unter denen des darüberstehenden Wassers. Der Grund dafür ist in der $\mathrm{O}_{2}$-Zehrung des Sedimentes zu sehen, die bei der nur noch schwachen Belastung stärker ins Gewicht fällt als die Zehrung im Wasserkörper.

\section{Der aktuelle Sauerstoffgebalt in der Sediment-Wasser-Kontaktzone unter dem Einfluß des Abwassers}

Die Infauna ist von der Sediment-Wasser-Kontaktzone in hohem Maße abhängig, da alle Röhren und Bauten - mit einigen Ausnahmen - hier enden und die Organismen aus 
dieser Wasserschicht den zur Atmung benötigten Sauerstoff beziehen. Deshalb erschien es sinnvoll, den Tagesgang des Sauerstoffs in dieser Zone bei Abwassereinfluß im Vergleich zu einer unbelasteten Kontrollstation aufzunehmen. Anhand von Dauermessungen an mehreren Stationen über einen Zeitraum von $48 \mathrm{~h}$ sollte gezeigt werden, welchen Einfluß die Zehrung des Abwassers sowie der sedimentierten partikulären organischen Substanzen auf diese Grenzschicht hat.

Die Auswahl der Meßstationen erfolgte so, daß die $\mathrm{O}_{2}$-Elektrode in kleine, flache Rinnsäle placiert werden konnte, damit sie möglichst auch bei Niedrigwasser vom Wasser umspült wurde und damit eine, für genaue Messungen benötigte, minimale Strömung vorhanden war.

Tabelle 3

Sauerstoffmessungen in der Sediment-Wasser-Kontaktzone über zwei Tidenzyklen im Untersuchungsgebiet bei Vancouver. Zeiten in $\%$ von $48 \mathrm{~h}$, während der die Kontaktzone (a) sauerstofffrei war, (b) in der der Sauerstoff Werte bis max. $1 \mathrm{mg} / 1 ; 2 \mathrm{mg} / \mathrm{l}$ und $4 \mathrm{mg} / \mathrm{l}$ erreichte und (c) in der höhere Werte als $4 \mathrm{mg} / 1$ gemessen wurden. Zusätzlich angegeben werden die Minimal- und Maximalwerte des gelösten Sauerstoffs, die Wassertemperaturen und Wasserbedeckungszeiten während der Messungen und der Anteil an organischer Substanz im Sediment der Meßstationen. $\mathbf{K}=$ Kontrollstation

\begin{tabular}{|c|c|c|c|c|c|c|}
\hline \multirow[t]{2}{*}{ Bedingungen } & & \multicolumn{5}{|c|}{$\begin{array}{l}\text { Bezeichnung der Probenstationen und } \\
\text { ihre Entfernungen von der Einleitung (m) }\end{array}$} \\
\hline & & $\begin{array}{c}1 \\
650\end{array}$ & $\begin{array}{c}2 \\
950\end{array}$ & $\begin{array}{c}3 \\
1200\end{array}$ & $\begin{array}{c}4 \\
1550\end{array}$ & $\underset{3000}{\mathrm{~K}}$ \\
\hline \multirow{5}{*}{$\begin{array}{l}\text { Prozentuale } \\
\text { Zeit von } 48 \\
\text { Std bei } \\
\text { Sauerstoff- } \\
\text { gehalten von: }\end{array}$} & $\begin{array}{c}0 \\
\mathrm{mg} / 1\end{array}$ & 15,1 & 3,6 & 44,0 & 0 & 0 \\
\hline & $\begin{array}{l}\leq 1 \\
\mathrm{mg} / 1\end{array}$ & 19,8 & 7,8 & 62,5 & 0 & 0 \\
\hline & $\begin{array}{l}\leq 2 \\
\mathrm{mg} / 1\end{array}$ & 32,8 & 7,8 & 77,6 & 0 & 0 \\
\hline & $\begin{array}{l}\leq 4 \\
\mathrm{mg} / 1\end{array}$ & 58,3 & 41,0 & 81,3 & 0,8 & 0 \\
\hline & $\begin{array}{l}>4 \\
\mathrm{mg} / 1\end{array}$ & 41,7 & 59,0 & 18,7 & 99,2 & 100,0 \\
\hline \multirow{4}{*}{$\begin{array}{l}\text { Minimum } \mathrm{O}_{2} \\
\text { Maximum } \mathrm{O}_{2} \\
\text { Uberflutungszeit } \\
\text { Wassertemperatur } \\
\text { organische Substanz } \\
\text { im Sediment }\end{array}$} & $(\mathrm{mg} / \mathrm{l})$ & 0 & 0 & 0 & 2,4 & 6,2 \\
\hline & $\begin{aligned}(\mathrm{mg} / \mathrm{l}) & \end{aligned}$ & $\begin{array}{r}8,6 \\
100\end{array}$ & $\begin{array}{r}7,2 \\
1000\end{array}$ & 6,4 & $\begin{array}{r}8,6 \\
75\end{array}$ & 10,0 \\
\hline & $\begin{array}{c}(\% / 48 \mathrm{~h}) \\
\left({ }^{\circ} \mathrm{C}\right)\end{array}$ & $\begin{array}{l}100,0 \\
5-10\end{array}$ & $\begin{array}{l}100,0 \\
8-14\end{array}$ & $\begin{array}{l}100,0 \\
8-12\end{array}$ & $\begin{array}{l}75,0 \\
6-10\end{array}$ & $10-17$ \\
\hline & $(\%)$ & 15,2 & 12,6 & 17,8 & 9,2 & 4,7 \\
\hline
\end{tabular}

Die Ergebnisse dieser in situ-Messungen sind in Tabelle 3 und Abbildung 7 zusammengefaßt. Sie zeigen, daß es im Bereich der Einleitungsstelle häufig und für längere Zeit zu anaeroben Verhältnissen in der Kontaktzone (bis zu $44 \%$ der Zeit) kam, und daß der Abwassereinfluß an allen Meßstationen - mit Ausnahme der Kontrollstation - zu niedrigeren $\mathrm{O}_{2}$-Werten als $4 \mathrm{mg} / \mathrm{l}$ führte. Dieser Wert wird als Minimum angesehen, um irreparable Schäden in einem Biotop zu vermeiden (Georgia Water Control Research, 1967;

Abb. 7: Untersuchungsgebiet Vancouver. Sauerstoffgehalt in der Sediment-Wasser-Kontaktzone. Kontinuierliche Aufzeichnung der Werte von vier Meßstationen $50 \mathrm{~m}$ neben dem Abwasserkanal sowie von einer Kontrollstation (Südmole) über 48 Stunden. Entfernungen der Stationen von der Einleitungsstelle vgl. Tabelle $3 . \mathrm{H}=$ Hochwasser; $\mathrm{N}=$ Niedrigwasser 


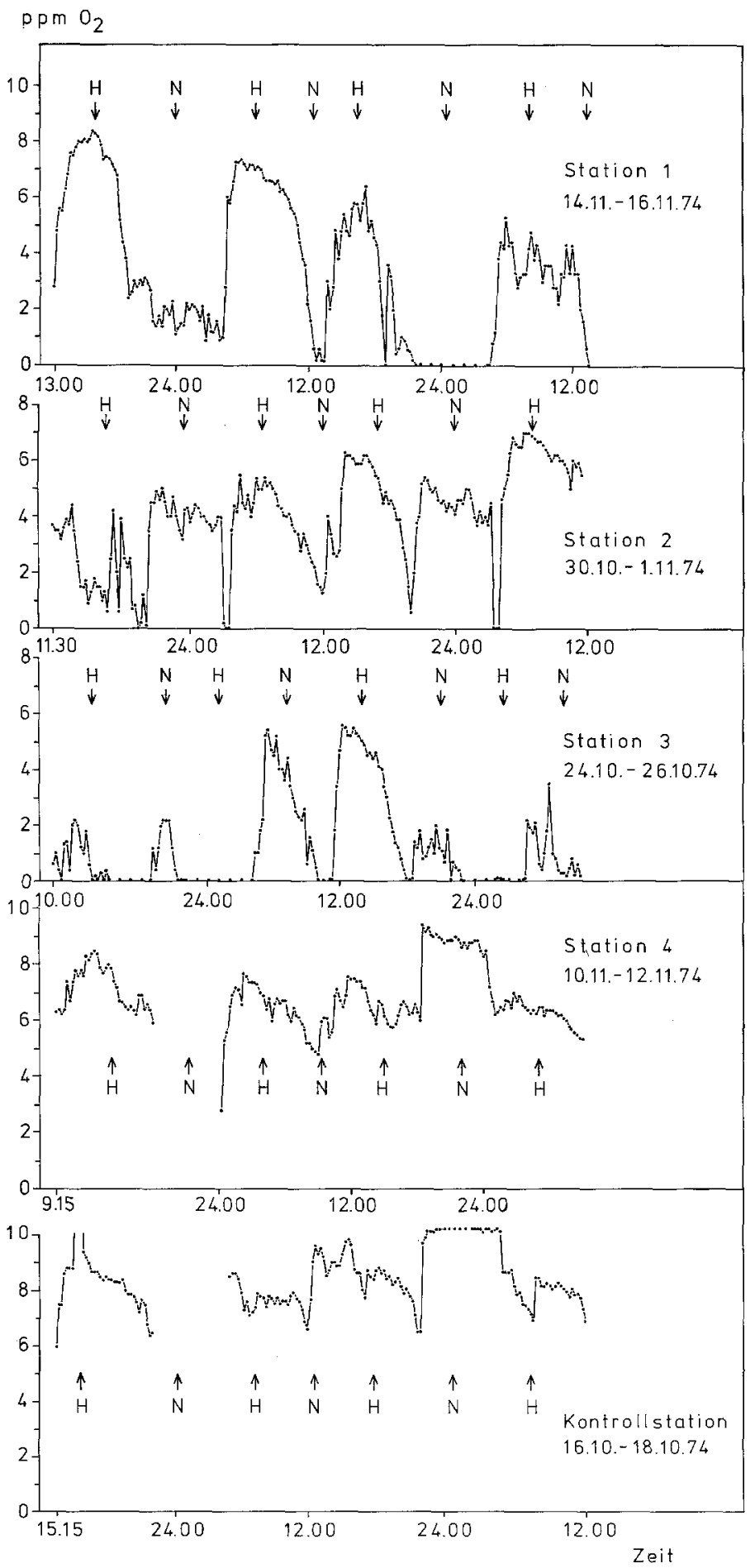


National Technical Advisory Commitee, 1968). Weiterhin wird deutlich, daß die $\mathrm{O}_{2}$ Zehrung nicht vom Verschmutzungsgradienten (als Entfernung von der Einleitungsstelle) abhing, sondern daß der Anteil des organischen Materials im Sediment den größten Einfluß auf die Zehrung in der Kontaktzone ausübte: Die niedrigsten $\mathrm{O}_{2}$-Werte wies die Station 3 auf, die sich durch den höchsten Detritusanteil im Sediment auszeichnete. Hinzu kommt, $\mathrm{daß}$ in dem gleichen Gebiet die höchsten Keimzahlen für Escherichia coli im Sediment gefunden wurden und nicht etwa näher zur Einleitungsstelle hin (B. C. Research, 1975). Dies beweist, daß bei der Station 3 die feinsten Abwasserpartikel sedimentieren konnten, die dann für die hohen Sauerstoffzehrungsraten verantwortlich waren. An der Kontrollstation, die sich am Ende der kleinen Südmole (s. Abb. 2) befand, wurden dagegen trotz höherer Wassertemperaturen während der Meßzeit niemals Werte von weniger als $6 \mathrm{mg} / \mathrm{l}$ registriert.

Aus den $\mathrm{O}_{2}$-Kurven (Abb. 7) ist ferner ersichtlich, daß die Sauerstoffminima besonders bei Station 2 und 3 - nicht etwa zu Zeiten geringster Strömung während der Hoch- und Niedrigwasserzeiten auftraten, sondern häufig dann, wenn die Strömungsgeschwindigkeit des auf- oder ablaufenden Wassers am größten war und man eigentlich wegen des schnellen Wasseraustausches und der Turbulenzen höchste $\mathrm{O}_{2}$-Werte erwarten sollte. Dieses Phänomen kommt dadurch zustande, daß bei steigender Strömungsgeschwindigkeit feine organische Partikel von der Sedimentoberfläche aufgewirbelt und in der Wassersäule verteilt werden. Dies führt zu einem schnellen $\mathrm{O}_{2}$-Verbrauch in der Kontaktzone und drückt den vorher relativ hohen Sauerstoffanteil innerhalb von 15 Minuten auf Null herunter. Dieser Vorgang konnte mehrere Male zu Zeiten höchster Strömungsgeschwindigkeiten beobachtet werden. Wird die Strömung wieder geringer, so sedimentieren die feinen Partikel; die intensive Zehrung in der Kontaktzone wird dadurch vermindert und der Gehalt an gelöstem Sauerstoff steigt schnell an.

\section{Abwasserbedingte Veränderungen des Salzgebaltes im Wattengebiet}

Ebenso wie die Beeinflussung des gelösten Sauerstoffs bleibt eine deutliche Erniedrigung des Salzgehaltes durch das Abwasser auf den relativ engen Einleitungsbereich beschränkt. Wie schon oben erwähnt, verdrängt das mit jeder Tide frisch einströmende Meerwasser das Abwasser wegen seiner geringen Dichte vom Boden, so daß das Benthos bei Überflutung keiner Salzgehaltserniedrigung ausgesetzt ist. Das Abwasser fließt jetzt an der Oberfläche $a b$, was zu einer deutlichen Schichtung im Wasserkörper führt. Messungen entlang des Abwasserkanals bei Hochwasser im Wattengebiet bei Vancouver zeigten eine starke Salinitätserniedrigung an der Oberfläche bei der Einleitungsstelle (Abb. 5). Seewärts tritt dann aber eine stärkere Durchmischung des Abwassers mit dem vom Meer nachdrängenden Seewasser ein, so daß sich der Salzgehalt an der Oberfläche dem des Bodenwassers angleicht.

Bei Niedrigwasser kommt es nur im Abwasserpriel zu einer Aussüßung der Sedimentoberfläche. Wo das Abwasser - wie in den Sylter Untersuchungsgebieten - bei Niedrigwasser in einem flachen Priel abläuft, wirkt es im oberen Bereich des Prieles konzentriert 
auf das Sediment ein (s. Salzgehaltwerte und Abwasserkonzentration in Tab. 2). Im Priel bei Kampen war erst nach $200 \mathrm{~m}$ Fließstrecke eine deutliche Erhöhung der Salzgehaltwerte durch Verdünnung des Abwassers mit Meerwasser zu verzeichnen. Entsprechend stieg der Salzgehalt dann seewärts rasch weiter an, während die Konzentration des Abwassers im gleichen Verhältnis abnahm.

Betrachtet man lediglich die Salinitätserniedrigung als Maß für den Abwassereinfluß, so ergibt sich nur ein ungenaues Bild; die Zeit, in der ein niedriger Salzgehalt auf das Sediment einwirkt, muß ebenfalls berücksichtigt werden. Ein Abwasserbelastungsfaktor, der sich aus der Abwasserkonzentration und ihrer Einflußzeit an einer Untersuchungsstation ergibt, eignet sich daher besser.

Es wird dabei von der Voraussetzung ausgegangen, daß sich während der gesamten Niedrigwasserzeit der Salzgehalt an der betreffenden Station nicht ändert. Dies war jedoch nicht an allen Stationen der Fall. Die Ergebnisse stellen folglich nur Durchschnittswerte dar.

Am Beispiel des Untersuchungsgebietes bei Kampen konnte durch Berechnung dieses Faktors gezeigt werden, daß der Abwassereinfluß nicht linear mit der Entfernung von der Einleitungsstelle abnimmt (Tab. 2). Ein starker Einfluß des Abwassers (und damit des erniedrigten Salzgehaltes) besteht bis zur 150-m-Marke (Station K 3): 0,8 bis 0,46 (wobei 1,0 reines Abwasser über 24 Std. bedeuten würde). Dann wird er auf fast die Hälfte reduziert $(0,26)$, um von der 300-m-Marke an (Station K 6) so gering zu werden, daß er in bezug auf die euryvalente $W$ attenfauna vernachlässigt werden kann.

In Vancouver wird das Abwasser in einem rund $2 \mathrm{~m}$ tiefen Kanal bei Niedrigwasser abgeleitet. Messungen des Salzgehaltes zeigten, daß in diesem Kanal auch bei Niedrigwasser das Meerwasser nicht vollständig abläuft und dadurch lediglich im Oberflächenwasser der Salzgehalt signifikant erniedrigt wird, und zwar von der Einleitungsstelle bis zur Niedrigwasserlinie (Abb. 5). Nur im mittleren Abschnitt des Kanals, wo ein größerer Wattenpriel einmündet, ist die Salinität auch an der Wasseroberfläche deutlich erhöht.

Um festzustellen, ob eventuell das Abwasser auch in das Sediment eindringt, wenn es in hohen Konzentrationen darüber hinwegfließt, wurde im Untersuchungsgebiet bei Kampen/Sylt das Sedimentwasser an den Stationen auf den Wattflächen und im oberen Bereich des Abwasserpriels in $5 \mathrm{~cm}$ Tiefe untersucht. Die Ergebnisse zeigen, daß nicht von einer merklichen Salinitätserniedrigung im Sediment der Wattflächen gesprochen werden kann (Tab. 5). Zu keiner Zeit und an keiner Station fiel der Salzgehalt unter $26 \%$. Er lag durchschnittlich sogar bei 28-29\%. Ein Eindringen des Abwassers ins Sediment fand also praktisch nicht statt. Selbst im Sediment des oberen Abwasserprieles war der Salzgehalt nur von der Einleitungsstelle bis zur 25-m-Marke auf 6,2 bis $9,6 \%$ herabgesetzt, während das Prielwasser zur gleichen Zeit einen Salzgehalt zwischen 2,3 und 2,5\% aufwies. $50 \mathrm{~m}$ von der Einleitungsstelle entfernt wurde im Sediment bereits $24,6 \%$ gemessen; nach $100 \mathrm{~m}$ stieg die Salinität auf 27,8\% und nach $200 \mathrm{~m}$ auf $28,3 \%$ an.

$\mathrm{Zu}$ erklären ist diese Beobachtung dadurch, daß bei Hochwasser salzhaltiges Meerwasser bis zur Einleitungsstelle über dem Sediment steht (s. o.). Da unter dem Oberflächensediment eine wasserundurchlässige Kleischicht eingezogen ist, wird das Meerwasser auch bei Niedrigwasser vom Sediment festgehalten. Es verhindert wegen seiner höheren Dichte ein Eindringen des Abwassers. Erst zum Ufer hin, wo das Sediment von Grobsand gebildet wird, kann das Meerwasser bei Niedrigwasser absinken und Abwasser nachlaufen. 


\section{Abwassereinfluß auf den $p H-W e r t$}

Der $\mathrm{pH}$-Wert wird im Vorfluter-Watt noch weniger als der Sauerstoffgehalt und der Salzgehalt vom Abwasser beeinflußt. In Kampen floß das Abwasser mit einem durchschnittlichen $\mathrm{pH}$-Wert von 7,5 in das Watt ein (Tab. 2). Er stieg schon nach $100 \mathrm{~m}$ auf einen Wert von 8,1 an, was durch die Assimilation der benthischen Mikroalgen bedingt war. Dieser Wert liegt für Seewasser bereits im Normalbereich. Weiter seewärts stieg der $\mathrm{pH}$-Wert allerdings noch bis auf 8,9 an. Derart hohe Werte sind in erster Linie auf die Assimilationstätigkeit der Seegraswiesen auf den benachbarten Wattflächen zurückzuführen, aus denen bei Ebbe Seewasser in den Abwasserpriel strömte. Es konnten an sonnigen Tagen sogar Maximalwerte von $\mathrm{pH}$ 9,5 gemessen werden.

Im Abwasserkanal bei Vancouver lagen die $\mathrm{pH}$-Werte generell etwas niedriger als im Sylter Untersuchungsgebiet, da wegen der hier eingeleiteten größeren Abwassermengen der Einfluß der benthischen Mikroalgen naturgemäß nur gering war. Er schwankte zwischen 6,9 und 8,4 mit durchschnittlich etwas niedrigeren Werten an der Einleitungsstelle und am Boden.

Selbst im Sedimentwasser kommt es - etwa durch starke Fäulnisprozesse - nur zu einer geringen pH-Erniedrigung. Tabelle 5 zeigt die Werte im interstitiellen Wasser an den Wattstationen bei Kampen im Verhältnis zu den pH-Werten im Oberflächenwasser kleiner Wattpfützen. Ein Einfluß des Abwassers war nur dadurch nachzuweisen, daß an den näher zum Abwasserpriel liegenden Stationen die Werte durchschnittlich unter denen der nachfolgenden Stationen lagen.

\section{Einfluß des Abwassers auf den Trübstoffgebalt im Einleitungsbereich}

Bei schlechter mechanischer Abwasserklärung werden große Mengen an Trübstoffen ins Wattenmeer eingeleitet. Durch Absorption des Lichtes können sie die Photosynthese der benthischen Mikroalgen beeinträchtigen. Gleichzeitig führt der Anteil an organischer Substanz im Seston zu einer Erhöhung des Nährstoffangebotes für Detritusfresser der benthischen Fauna. Da das Wattenmeer schon sehr trübstoffreich ist, sollte untersucht werden, wie stark sich der Gesamtsestongehalt und sein Anteil an organischer Substanz durch die zusätzliche Einleitung von Trübstoffen verändern.

Im Wattengebiet von Vancouver wurden daher im Abwasserkanal der Trübstoffgehalt des Oberflächen- und Bodenwassers sowohl bei Niedrigwasser als auch bei Hochwasser gemessen. Abbildung 8 zeigt, daß die höchsten Seston-Werte jeweils im oberen Abschnitt des Abwasserkanals gefunden wurden, wobei die Werte in Bodennähe deutlich über denen der Oberfläche lagen. Daraus kann geschlossen werden, daß es sich beim Seston in den unteren Wasserschichten in erster Linie um aufgewirbeltes Bodensediment handelt. Dies wird bestätigt bei einer Analyse des Sestons: Der prozentuale Anteil des Detritus am Gesamtseston war an der Oberfläche, wo sich das Abwasser einschichtete (s. o.), sowohl bei Hoch- als auch bei Niedrigwasser sehr viel höher als am Boden. Folglich stammt der Detritus aus dem Abwasser.

Während er in der Oberflächenschicht seewärts abnahm, stieg der Detritusanteil am Boden mit zunehmender Entfernung leicht an. Die organischen Abwasserbestandteile 


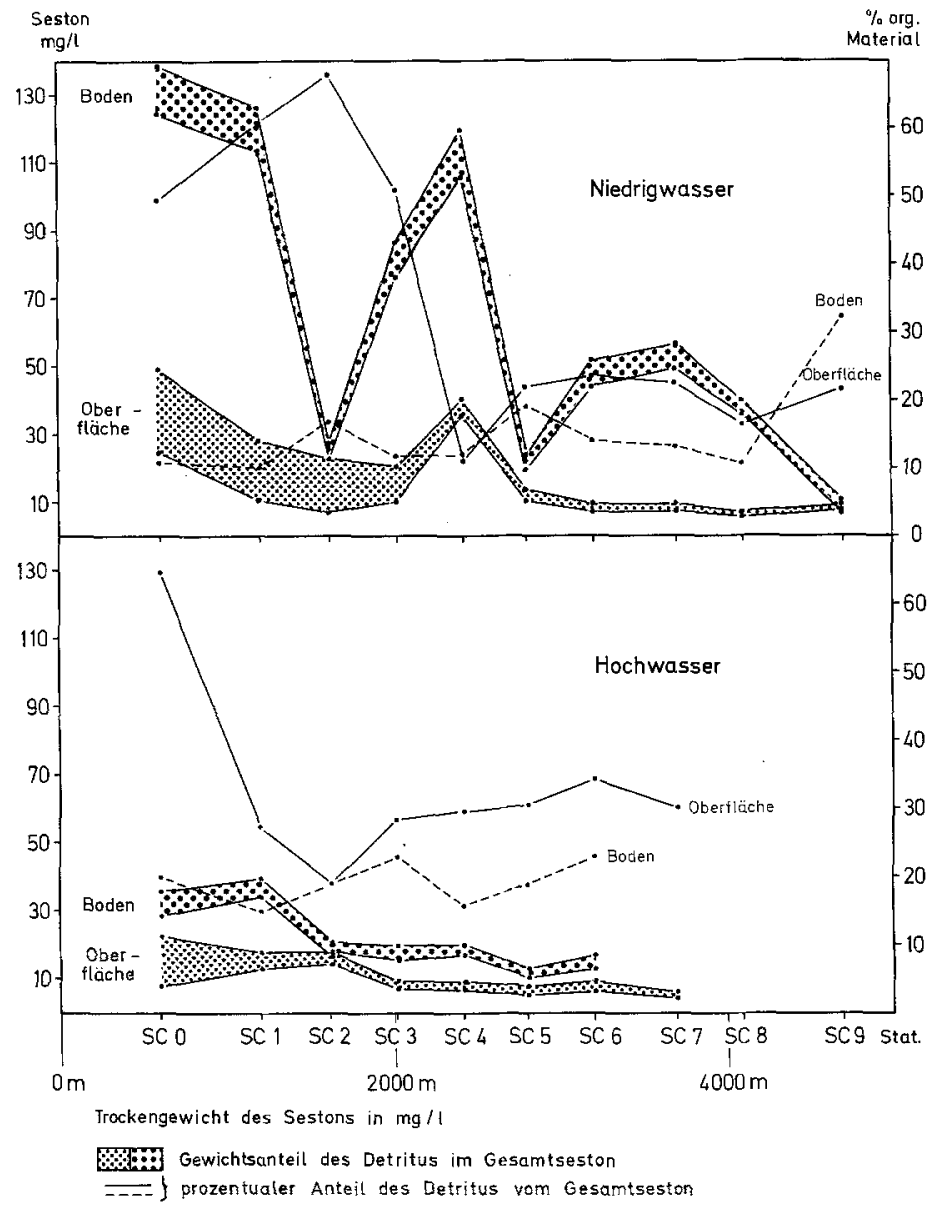

Abb. 8: Untersuchungsgebiet Vancouver. Der Sestongehalt im Abwasserkanal in Abhängigkeit von den Tiden. Werte von der Wasseroberfläche und vom Boden. Die gepunkteten Flächen zeigen den absoluten Anteil des Detritus im Seston

sedimentierten also aus. Bei Niedrigwasser wurden sie mit der Strömung im Abwasserkanal wesentlich weiter seewärts transportiert als bei Hochwasser. Der generell höhere Sestongehalt bei Niedrigwasser ist ebenfalls durch die Strömung im Kanal bedingt. Der erneute steile Anstieg der Seston-Werte nach etwa $2500 \mathrm{~m}$ Fließstrecke wird dagegen durch die Einmündung eines größeren Wattenprieles verursacht.

Die Untersuchungen zeigen, daß durch das Abwasser größere Mengen Detritus ins Wattenmeer gespült werden, die bei Hochwasser im oberen Wattenbereich sedimentieren, während sie bei Niedrigwasser mit dem ablaufenden Meerwasser weiter über das Watt transportiert werden. Dadurch kommt es zu einer teilweisen Verteilung der vom Makrobenthos verwertbaren organischen Substanzen über die Wattflächen. 
Abbau der gelösten organischen Koblenstoffverbindungen des Abwassers im Watt

Der gelöste organische Kohlenstoffgehalt - als Maß für die gelösten organischen Verbindungen - wurde nur zweimal während der Voruntersuchungen im Abwasserpriel bei Kampen/Sylt gemessen.

Wie zu erwarten, nahm er von der Einleitungsstelle zur Niedrigwasserlinie hin kontinuierlich ab (Tab. 2). Dabei scheint die Abnahme des Kohlenstoffgehaltes im oberen Abschnitt des Prieles bis etwa zur 200-m-Marke durch intensive Abbauvorgänge verursacht worden zu sein. Eine Verdünnung des Abwassers durch zufließendes Seewasser konnte hier vernachlässigt werden. Im weiteren Prielverlauf war dann jedoch nicht mehr zwischen einem Verdünnungseffekt und einem Abbau zu unterscheiden, da der Kohlenstoffgehalt des zufließenden Meerwassers nicht bekannt war.

\section{Veränderungen der Sedimente unter Abwassereinfluß}

\section{Verteilung der Korngrößen}

Eine Abwassereinleitung hat keinen direkten Einfluß auf die Verteilung der Korngrößen. Sie hängt vielmehr von den Strömungsverhältnissen im Wattengebiet und von der Genetik der Sedimente unter der Oberflächenschicht ab. Dies trifft insbesondere für die Sedimente im Abwasserpriel des Untersuchungsgebietes bei Kampen zu. So wurden hier die für Schlickwatten recht hohen Medianwerte der Korngrößen durch den groben Dünensand unter der nur sehr dünnen Schlickschicht und durch den geringen Anteil der Silt-Ton-Fraktion verursacht (Tab. 4). Die Verteilung der feinen Fraktionen (Silt und Tone) ist dabei eine direkte Folge der unterschiedlichen Strömungsgeschwindigkeiten des

Tabelle 4

Sedimentfaktoren im Abwasserpriel von Kampen (Sylt). *: Medianwerte

\begin{tabular}{|c|c|c|c|c|c|c|c|c|c|}
\hline Komponenten & $\begin{array}{c}\mathrm{K} 0 \\
0\end{array}$ & $\begin{array}{c}\text { und } \\
\mathrm{K} 1 \\
50 \\
\end{array}$ & $\begin{array}{c}\text { re Ent } \\
\text { K } 2 \\
100 \\
\end{array}$ & $\begin{array}{c}\text { Probe } \\
\text { aunger } \\
\text { K 3 } \\
150 \\
\end{array}$ & $\begin{array}{c}\text { atione } \\
\text { on der } \\
\text { K } 4 \\
200 \\
\end{array}$ & $\begin{array}{c}\text { Einlei } \\
\text { K } 6 \\
300\end{array}$ & $\begin{array}{c}\text { ingsste } \\
\mathrm{K} 8 \\
400\end{array}$ & $\begin{array}{l}\text { le } \\
\text { K } 10 \\
500\end{array}$ & $\begin{array}{r}\mathrm{K} 12 \\
600 \mathrm{~m}\end{array}$ \\
\hline \multirow{4}{*}{$\begin{array}{l}\text { Oxydations- } \\
\text { schicht }(\mathrm{cm}) \\
\text { Korngrößen- } \\
\text { verteilung }(\mu \mathrm{m})^{*} \\
\text { Silt-Ton-Anteil }(\%) \\
\text { organische } \\
\text { Substanz }(\%)\end{array}$} & 0,0 & $\begin{array}{c}0- \\
0,1\end{array}$ & $\begin{array}{l}0,1- \\
0,5\end{array}$ & $\begin{array}{l}0,5- \\
1,0\end{array}$ & 1,0 & $\begin{array}{l}1,0 \\
3,0\end{array}$ & $\begin{array}{l}1,0 \\
2,0\end{array}$ & $\begin{array}{l}1,0- \\
2,0\end{array}$ & $\begin{array}{l}1,0- \\
1,5\end{array}$ \\
\hline & 310 & 270 & 260 & 265 & 265 & 280 & 285 & 270 & 235 \\
\hline & 5,3 & 2,4 & 1,9 & 2,0 & 0,4 & 0,1 & 0,5 & 0,7 & 2,4 \\
\hline & 2,9 & 1,9 & 0,8 & 1,0 & 0,6 & 0,5 & 0,8 & 0,7 & 0,9 \\
\hline
\end{tabular}

ablaufenden Wassers zwischen Einleitungsstelle und TNW-Linie: Im oberen Abschnitt (0-180 m) floß das Abwasser ohne deutlichen Priel in breiter Fläche über das Sediment. Dadurch wurden die hier bei Hochwasser abgelagerten feineren Korngrößen nur zu einem 


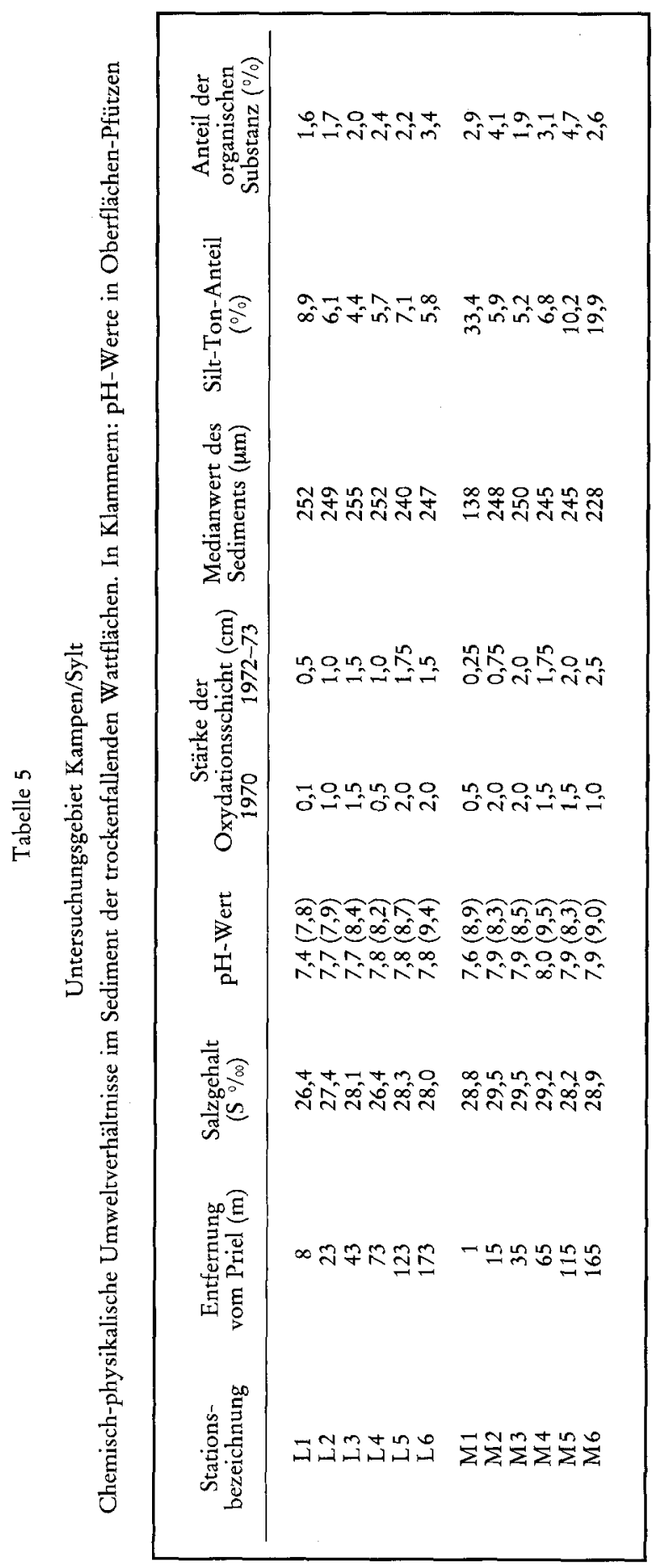


geringen Teil wieder ausgeschwemmt. Die erhöhten Anteile der Silt-Ton-Fraktion beweisen dies. Der mittlere Abschnitt (200-500 m) war durch ein tieferes Prielbett (max. 0,5 m Tiefe) und höhere Strömungsgeschwindigkeiten gekennzeichnet und wies daher nur extrem niedrige Werte für die Silt-Ton-Anteile auf. Im Bereich der Niedrigwasserlinie mündete der Priel in eine breite Wasserfläche ein. Die Fließgeschwindigkeit wurde dabei wieder herabgesetzt und erlaubte dadurch einen höheren Silt-Ton-Anteil im Sediment.

Auf den Wattflächen war der Schlammgehalt höher als im Abwasserpriel, wie aus den Werten für die Silt-Ton-Anteile ersichtlich ist (Tab. 5). Dadurch wurden auch die Medianwerte für die Verteilungen der Korngrößen herabgesetzt. Die höchsten Anteile der feinen Fraktionen wies das Sediment in beiden Transekten (L- und M-Transekt) in Prielnähe auf. Da es sich aber bei ihnen in erster Linie um anorganische Sedimentbestandteile handelte, wäre es reine Spekulation, dafür den Abwassereinfluß als Ursache anzuführen.

Im Untersuchungsgebiet bei List und Braderup läßt sich ebenfalls kein direkter Abwassereinfluß auf die Korngrößenzusammensetzung nachweisen. Hohe Medianwerte $(290-310 \mu \mathrm{m})$ und hohe Silt-Ton-Anteile $(8,7-13,2 \%)$ bei List zeigen, daß es sich um ein ehemaliges Grobsandgebiet handelt, das in der Aufschlickung begriffen ist. Die Aufschlikkung wird vornehmlich durch einen Dünenhaken verursacht, der sich halbkreisförmig um das Einleitungsgebiet gebildet hat. Eine Beschleunigung der Aufschlickung durch die Abwasserinhaltsstoffe ist allerdings wahrscheinlich.

Bei Braderup wird das Abwasser in ein reines Schlickgebiet ohne grobsandigen Untergrund geleitet. Das Sediment war durch niedrige Medianwerte $(55-70 \mu \mathrm{m})$ und hohe Silt-Ton-Anteile $(31,1-47,8 \%$ ) charakterisiert. Der Einfluß des Abwassers war wegen des natürlichen hohen Schlickgehaltes nicht zu quantifizieren.

Die Verteilung der Sedimente im Untersuchungsgebiet von Vancouver ließ mit Ausnahme des Abwasserkanals keine prinzipiellen Unterschiede gegenüber unbelasteten Wattengebieten erkennen (Pestrong, 1972). Nur der sehr hohe Anteil der feinen Fraktionen (Silt-Ton) im Bereich der Einleitungsstelle (nördlicher Teil des A-Transektes), an einigen Stationen im mittleren Wattabschnitt (B-Transekt) sowie im Abwasserkanal lassen sich durch das Einbringen von feinem partikulärem Material durch das Abwasser erklären (Tab. 6).

Wegen der Heterogenität des Untersuchungsgebietes soll die Verteilung der Sedimente ausführlicher beschrieben werden: Die Sedimente in Ufernähe waren zur Einleitungsstelle hin am schlickigsten (Stationen A 0-A 4) mit einem Medianwert kleiner als $63 \mu \mathrm{m}$ und einem hohen Silt-Ton-Anteil von 62,6\% bis 94,1\%. Die anderen Stationen des Transektes hatten gröbere Sedimente mit einer Silt-Ton-Fraktion von 33,6\% bis 56,2 \% mit Ausnahme von Station A 8 mit nur 8,7\% "feiner" Bestandteile. Die groben Sedimente aus dem Fraser River scheinen die Stationen dieses Transektes nicht zu beeinflussen, wie die Analysen von A 9 und A 10 zeigen. Im mittleren Wattabschnitt waren die Sedimente etwas grober, mit Ausnahme der Stationen B 4, B 5 und B 7. Diese Stationen liegen in flachen Senken und werden durch den Hauptpriel stärker vom Abwasser beeinflußt als die anderen Stationen. B 6 dagegen liegt direkt am Prielrand, wo wegen der höheren Strömungen gröberes Sediment dominiert.

Der C- und D-Transekt liegen in einem sandigen Wattabschnitt, woraus die durchschnittlich höheren Medianwerte und der geringere Silt-Ton-Anteil resultieren. 
Tabelle 6

Medianwerte der Korngrößenverteilungen sowie Anteile der Silt-Ton-Fraktion und der organischen Substanz der Sedimente im Untersuchungsgebiet bei Vancouver (Canada)

\begin{tabular}{|c|c|c|c|}
\hline Station & Medianwert $(\mu \mathrm{m})$ & Silt-Ton-Anteil $(\%)$ & organische Substanz $(\%)$ \\
\hline AO & $<63$ & 81,88 & 7,44 \\
\hline A 1 & $<63$ & 94,11 & 8,62 \\
\hline $\mathrm{A} 2$ & $<63$ & 67,96 & 3,00 \\
\hline A 3 & $<63$ & 62,67 & 4,18 \\
\hline A 4 & $<63$ & 85,36 & 2,86 \\
\hline A 5 & 55 & 42,75 & 1,94 \\
\hline A6 & $<63$ & 56,25 & 1,97 \\
\hline A7 & 74 & 40,59 & 2,03 \\
\hline A 8 & 90 & 8,76 & 1,29 \\
\hline A9 & 61 & 41,60 & 1,52 \\
\hline A 10 & 83 & 33,63 & 2,39 \\
\hline B1 & 97 & 9,06 & 1,90 \\
\hline B2 & 91 & 19,94 & 1,89 \\
\hline B3 & 95 & 21,92 & 2,07 \\
\hline B4 & 52 & 42,43 & 3,26 \\
\hline B 5 & 56 & 44,43 & 3,48 \\
\hline B6 & 108 & 11,0 & 1,64 \\
\hline B7 & $<63$ & 58,95 & 3,49 \\
\hline B 8 & 96 & 6,09 & 1,32 \\
\hline B9 & 99 & 6,17 & 1,53 \\
\hline B 10 & 88 & 7,12 & 1,65 \\
\hline C1 & 170 & 4,56 & 1,47 \\
\hline $\mathrm{C} 2$ & 135 & 5,3 & 1,59 \\
\hline C3 & 129 & 5,65 & 1,72 \\
\hline C4 & 91 & 15,74 & 2,13 \\
\hline C5 & 119 & 2,73 & 1,70 \\
\hline C6 & 108 & 15,97 & 1,78 \\
\hline C7 & 87 & 28,67 & 2,22 \\
\hline $\mathrm{C} 8$ & 108 & 2,12 & 1,31 \\
\hline $\mathrm{C} 10$ & 100 & 29,85 & 1,66 \\
\hline D 1 & 150 & 2,38 & 1,80 \\
\hline D5 & 172 & 0,79 & 1,65 \\
\hline D10 & 155 & 1,28 & 2,13 \\
\hline SC1 & $<63$ & 75,77 & 10,14 \\
\hline SC2 & $<63$ & 91,33 & 9,38 \\
\hline $\mathrm{SC} 3$ & 52 & 49,74 & 10,08 \\
\hline $\mathrm{SC} 4$ & 169 & 22,71 & 7,21 \\
\hline SC5 & 225 & 10,22 & 3,46 \\
\hline SC 6 & 280 & 3,19 & 2,67 \\
\hline $\mathrm{SC7}$ & 232 & 1,98 & 1,97 \\
\hline SC 8 & $<63$ & 91,79 & 3,11 \\
\hline SC9 & $<63$ & 82,23 & 1,77 \\
\hline
\end{tabular}

Die Sedimente im Abwasserkanal waren im oberen Kanalabschnitt sehr fein und wurden seewärts gröber. Bei den Stationen SC 8 und SC 9 allerdings war das Sediment ebenso fein wie an der Einleitungsstelle: $91,7 \%$ bzw. 82,2\% Silt-Ton-Anteil. Der Grund ist darin zu sehen, daß dem Abwasserkanal am Molenende eine Sandbank vorgelagert ist, wodurch im davorliegenden tieferen Prielabschnitt eine Art "Absetzbecken" für feinere Abwasser- und Sedimentpartikel entsteht; dieses führt zu der Dominanz der Silt-TonFraktion. 


\section{Anteil der organischen Substanz im Sediment}

Auch der Detritusanteil im Sediment läßt sich nur bedingt zum Nachweis eines Abwassereinflusses heranziehen. Die Werte im Abwasserpriel bei Kampen liegen meistens unter $1 \%$; lediglich an der Einleitungsstelle wurden 2,9 und 1,9\% erreicht (Tab. 4).

Die auf den Wattflächen generell höheren Anteile an partikulärem organischem Restmaterial im Vergleich zum Priel zeigen, daß die Abwasserpartikel in erster Linie auf den Wattflächen sedimentierten (Tab. 5); eine räumliche Abhängigkeit von der Einleitungsstelle des Abwassers war jedoch nicht nachzuweisen.

Auch im Untersuchungsgebiet bei List und Braderup konnte kein Zusammenhang zwischen der Verteilung des organischen Sedimentanteils und der Abwassereinleitung festgestellt werden. Im Verhältnis zu den hohen Werten der Silt-Ton-Fraktion war in beiden Wattengebieten nur wenig partikuläre organische Substanz im Sediment vorhanden $(\max .2,3 \%$ bzw. $7,3 \%$ ).

Die Ergebnisse der Detritus-Analysen im Watt bei Vancouver (Tab. 6) zeigen im großen und ganzen eine normale Verteilung, wie sie auch in unbelastetem Wattengebiet gefunden werden kann (Pestrong, 1972): Die höchsten Anteile wies das Sediment in Küstennähe und in tiefergelegenen Abschnitten des B-Transektes auf. Hier machte sich der Einfluß des natürlichen Wattenpriels bemerkbar. Die bei weitem höchsten Anteile an organischer Substanz wurden jedoch bei den Stationen in der Nähe der Einleitungsstelle (Station A O und A 1) und im oberen Abwasserkanal (Station SC 1-SC 4) gefunden.

\section{Beeinflussung der Diffusionstiefe von Sauerstoff im Sediment}

Die Eindringtiefe des Sauerstoffs in das Sediment - gemessen von der Oberfläche bis zur FeS-haltigen Reduktionsschicht - hängt unter anderem vom Sauerstoffangebot an der Oberfläche und der Zehrung in der oberen Sedimentschicht ab. Da nachgewiesen werden konnte, daß es in Wattengebieten nur im unmittelbaren Einleitungsbereich eines Abwasserablaufes zu einer $\mathrm{O}_{2}$-Erniedrigung im Wasser kommt, gibt die Messung der Schichtdicke des oxydierten Sedimentes Anhaltspunkte für die Stärke der aeroben Abbauaktivitäten (bzw. der Sauerstoffzehrung) an der Sedimentoberfläche.

Die Werte in Tabelle 4 und 5 für das Untersuchungsgebiet Kampen zeigen deutlich eine Abhängigkeit vom Abwassereinfluß. Im Verschmutzungszentrum des Prieles war das Sediment bis an die Oberfläche reduziert; eine oxydierte Oberflächenschicht zeigte sich erst nach $50 \mathrm{~m}$ und nahm seewärts nur langsam nach $200 \mathrm{~m}$ auf $1 \mathrm{~cm}$ Dicke zu.

Auf den W a t $\mathrm{fl}$ läch e n war diese Schicht in Prielnähe ebenfalls deutlich dünner als an den nachfolgenden Stationen.

Aus beiden Ergebnissen läßt sich auf dichte Bakterienrasen an der Sedimentoberfläche im Bereich hoher Abwasserkonzentrationen schließen. Diese Rasen verbrauchen den Sauerstoff schon an der Oberfläche ganz oder zum größten Teil und verhindern damit ein tieferes Diffundieren des $\mathrm{O}_{2}$ in das Sediment. Hinzu kommt noch ein höherer Anteil feiner Sedimentfraktionen in diesen Bereichen (Tab. 4 und 5), der ebenfalls die $\mathrm{O}_{2}$-Diffusion in tiefere Sedimentschichten erschwert. Zugleich erlauben feine Sedimente eine höhere Bakterienbesiedlung als Grobsedimente (Krumbein, 1971). Dies führt bei ausreichendem Ange- 
bot an abbaubaren organischen Substanzen zu einer erhöhten $\mathrm{O}_{2}$-Zehrungsrate des Sedimentes und damit zu einer geringeren Diffusionstiefe des Sauerstoffs. Der Abbau der durch das Abwasser importierten und abgelagerten organischen Substanzen muß in starkbelasteten Bereichen daher überwiegend anaerob erfolgen.

\section{Eutrophierung der Sedimente durch Abwassereinleitungen}

Der Grad der Eutrophierung in abgegrenzten oder abgeschlossenen Gewässern kann an der Höhe der Phosphat- und Nitratwerte des Wassers erkannt werden. In Tidengebieten, die mit dem offenen Meer einen intensiven Wasseraustausch haben, läßt sich der Eutrophierungszustand auf Grund der ständigen Veränderung aller wichtigen hydrochemischen Parameter nur großräumig unter Berücksichtigung des gesamten Küstengebietes mit Hilfe chemischer Wasseranalysen abschätzen (Hoos \& Packmann, 1974).

Um lokale Eutrophierungseffekte in Wattengebieten quantifizieren zu können, bietet sich als Möglichkeit der indirekten und schnellen Erfassung die Analyse des sedimentären Chlorophyll a in den Zonen unterschiedlicher Abwasserbelastungen an. Durch parallele Analyse des Phaeophytin a kann zusätzlich zwischen noch aktivem Chlorophyll a lebender Algen und seinen - inaktiven - Abbauprodukten unterschieden werden, wobei hohe Phaeophytin a-Anteile im Verhältnis zum Chlorophyll a Hinweise auf erhöhte Sterblichkeit der Algengesellschaften geben.

\section{Der Chlorophyll-Gebalt im Oberflächensediment in Abhängigkeit von der Abwasserbelastung}

In beiden Untersuchungsgebieten (Vancouver und Kampen/Sylt) war anhand der Chlorophyll-Analysen ein Einfluß des Abwassers auf die Biomasse der benthischen Mikroalgen nachzuweisen. Wie Abbildung 9 zeigt, war im Wattengebiet von Vancouver der Einfluß am deutlichsten im Uferbereich festzustellen (A-Transekt). In der Nähe des Abwasserkanals lag der Chlorophyll a-Gehalt des Sedimentes mit 99,9 $\mu \mathrm{g} / \mathrm{cm}^{3}$ extrem hoch, fiel nach etwa $1350 \mathrm{~m}$ Entfernung vom Verschmutzungszentrum (Station A 4) auf ein Minimum ab $\left(6,6 \mu \mathrm{g} / \mathrm{cm}^{3}\right)$ und stieg nach $2530 \mathrm{~m}$ (Station A 9) erneut an. Zum Mittelarm des Fraser Rivers (s. Abb. 2) nahmen die Chlorophyll a-Werte wieder geringfügig $\mathrm{ab}$.

Im mittleren Wattenabschnitt war ein Eutrophierungseffekt anhand der ChlorophyllAnalysen nicht so deutlich zu zeigen, da in diesem Gebiet ein natürlicher Wattenpriel zusätzlich zum Abwasserkanal zur Wachstumsförderung der benthischen Mikroalgen führte. Dies äußerte sich in einem zweiten Maximum der Chlorophyll-Werte bei Station B 6. Auch der Mittelarm des Fraser Rivers hatte einen positiven Einfluß auf die Algen. Insgesamt lagen hier die Chlorophyll-Werte aber schon niedriger als im Uferbereich.

Auf den Sandflächen zur Niedrigwasserlinie hin war der Chlorophyll a-Gehalt im Sediment noch niedriger als im mittleren Wattenabschnitt; dennoch zeigte sich ein leichter Anstieg zum Abwasserkanal hin. 

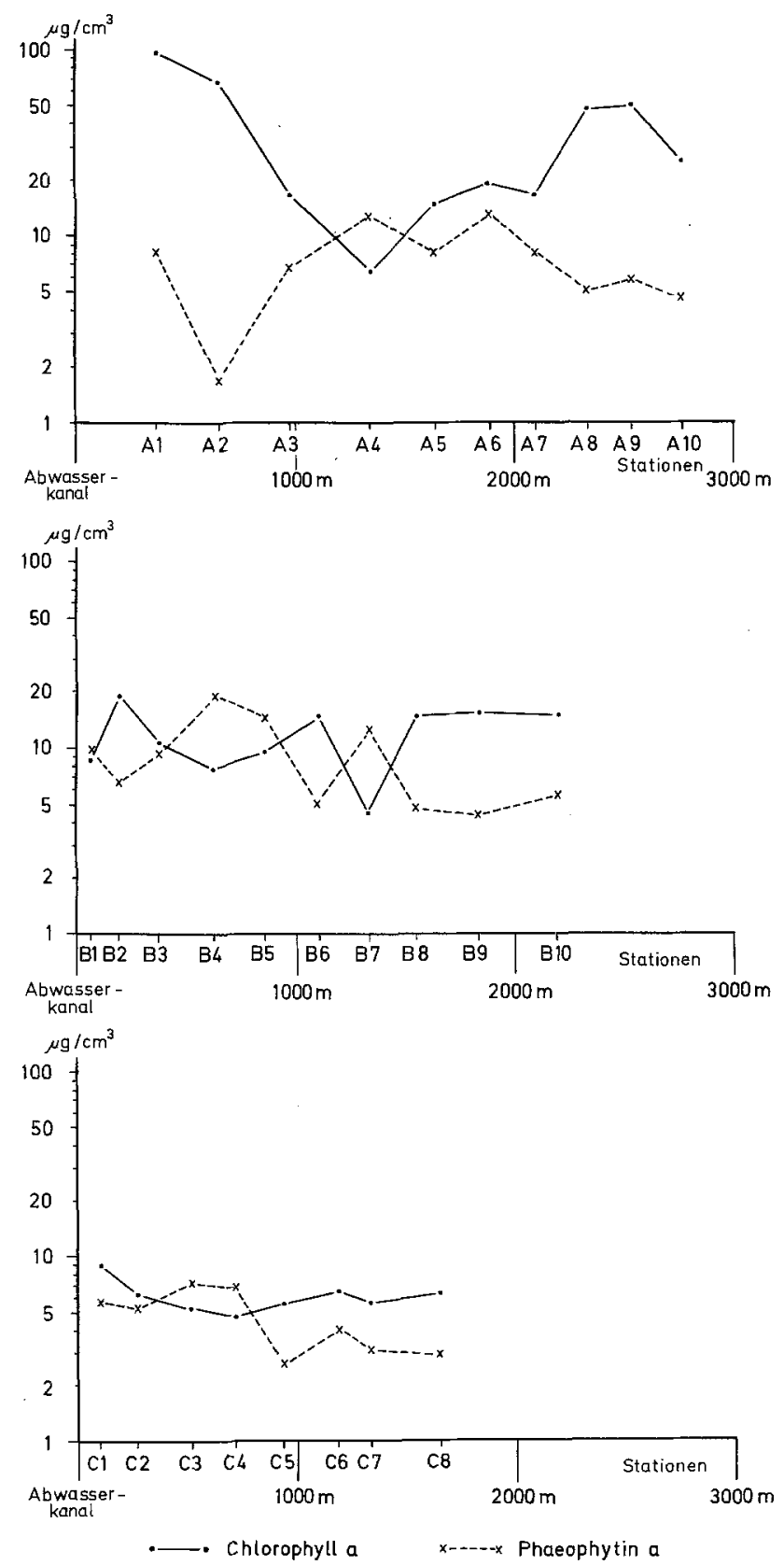

Abb. 9: Untersuchungsgebiet Vancouver. Chlorophyll a- und Phaeophytin a-Gehalt des Sedimentes auf den Wattflächen (0-1 cm Tiefe). Halblogarithmische Darstellung 
Die Werte für inaktives, totes Chlorophyll a, das Phaeophytin a, zeigten an allen drei Transekten die gleiche Tendenz: In einer bestimmten Entfernung vom Abwasserkanal war der Phaeophytin a-Gehalt des Sedimentes größer als der Gehalt an aktivem Chlorophyll a. Diese Zonen fielen mit einem Wechsel der Algengesellschaften zusammen: Mikroskopische Untersuchungen von Sedimentproben zeigten, daß im Uferbereich und im mittleren Wattenabschnitt zum Abwasserkanal hin Cyanophyceen-Gesellschaften dominierter. Mit zunehmender Entfernung vom Kanal verschob sich die Artendominanz zu den Diatomeen hin. Die Ubergangsgebiete waren zugleich durch ein Minimum an Chlorophyll a im Sediment gekennzeichnet (Abb. 9). Diese Beobachtung weist darauf hin, daß die Ubergangszonen ein Gebiet mit hoher Algensterblichkeit darstellen.

Im Untersuchungsgebiet bei Kampen wird der eutrophierende Effekt des Abwassers auf die benthischen Mikroalgen-Populationen noch deutlicher: sowohl im Juni als auch im September wurden die weitaus höchsten Chlorophyll a-Werte in der stark belasteten Zone des oberen Priel-Abschnittes gefunden (Abb. 10). Der eutrophierende Einfluß ließ sich auch durch die im Vergleich zum Juni höheren Chlorophyll a-Werte im September nachweisen und muß als Folge einer durch den Tourismus erhöhten Abwassermenge interpretiert werden.

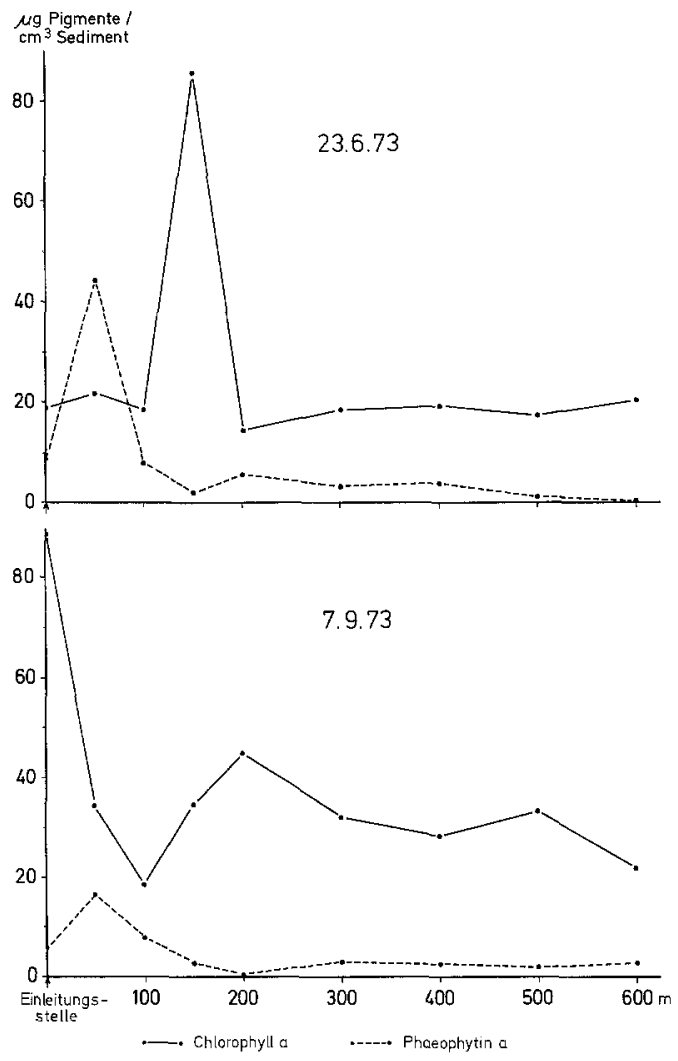

Abb. 10: Untersuchungsgebiet Kampen/Sylt. Chlorophyll a- und Phaeophytin a-Gehalt der Sedimentoberfläche im Abwasserpriel. 
Wie im Einleitungsgebiet von Vancouver besiedelten auch hier CyanophyceenGesellschaften den Einleitungsbereich (bis maximal $200 \mathrm{~m}$ von der Einleitungsstelle entfernt). Im Juni erreichten sie noch keine sehr hohen Individuendichten, während die Diatomeen, die etwa $150 \mathrm{~m}$ von der Einleitungsstelle entfernt die Blaualgen ablösten, schon ein Biomassen-Maximum entwickelten. Im September dagegen befand sich das Maximum der Cyanophyceen (gemessen als Pigmentmenge) direkt an der Einleitungsstelle, während das Diatomeen-Maximum bei der 200-m-Marke (Station K 4) zu finden war. Zwischen den beiden Maxima war eine Zone geringster Chlorophyll a-Werte festzustellen, die mit dem Wechsel der Algengesellschaften zusammenfiel. In diesem Bereich wurden - wie im Einleitungsbereich von Vancouver - wiederum die höchsten Werte an Phaeopigmenten gefunden (nur September). Im Juni lagen die höchsten Phaeophytin a-Werte innerhalb der Cyanophyceen-Gesellschaften und wurden - wie sich durch mikroskopische Untersuchungen ergab - zum Teil durch angespülte Zostera-Blätter verursacht.

Einen Hinweis auf den Abwassereinfluß gibt auch das Verhältnis von lebender zu toter Algenbiomasse ( $\mathrm{T}$ ab. 7 ): es war bei der hohen Abwasserkonzentration im oberen Prielabschnitt sehr stark zugunsten der abgestorbenen Algen verschoben. Nur direkt an

Tabelle 7

Untersuchungsgebiet Kampen/Sylt. Verhältnis von Chlorophyll a zu Phaeophytin a an der Sedimentoberfläche im Abwasserpriel

\begin{tabular}{|ccc|}
\hline Station & Juni 1973 & September 1973 \\
\hline K 0 & 2,09 & 14,93 \\
K 1 & 0,5 & 2,07 \\
K 2 & 2,35 & 2,4 \\
K 3 & 41,0 & 12,36 \\
K 4 & 2,6 & 112,25 \\
K 6 & 5,81 & 9,76 \\
K 8 & 5,0 & 10,88 \\
K 10 & 13,54 & 16,8 \\
K 12 & 68,67 & 7,52 \\
\hline
\end{tabular}

der Einmündung des Abwassergrabens in das Watt (Station $\mathrm{K} \mathrm{O}$ ) waren die Werte höher, weil hier ausschließlich die süßwasser-resistenten Cyanophyceen siedelten. Die sehr hohen Werte im mittleren Prielabschnitt weisen auf optimale Bedingungen für das Algenwachstum hin: gutes Nährstoffangebot bei vermindertem Abwassereinfluß. Im Anschluß an diese Zone fiel der Quotient auf mittlere Werte ab. Eine Ausnahme bildet nur der hohe Anteil von lebender Algenbiomasse an der Niedrigwasserlinie im Monat Juni (Station K 12). Er kann nur durch den äußerst geringen Phaeophytin a-Anteil bei etwa gleichbleibender Chlorophyll a-Menge (Abb. 10) erklärt werden und deutet auf den Beginn einer intensiven Algenwachstumsphase hin.

\section{Vertikalverteilung des Chlorophylls im Sediment in den Zonen unterschiedlicher Belastung}

Benthische Mikroalgen können aktiv Vertikalwanderungen im Sediment durchführen und dringen dabei bis zu $10 \mathrm{~cm}$ tief ein (Pamatmat, 1968; Cadee \& Hegeman, 1974). Es 
erschien daher sinnvoll zu untersuchen, ob kommunales Abwasser auf die Vertikalwanderungen bzw. auf ihre Vertikalverteilung im Sediment einen Einfluß hat. Dazu wurde im Untersuchungsgebiet Kampen (Sylt) die Verteilung von Chlorophyll a und Phaeophytin a im Sediment ermittelt.

Die Ergebnisse dieser Untersuchungen zeigen, daß die Menge an lebendem Chlorophyll generell an der Oberfläche am höchsten war (Abb. 11 und 12). Ein Zurückweichen der Algen in tiefere Sedimentschichten als Folge des Abwassereinflusses ließ sich nicht nachweisen. Zwar stieg im Bereich der Tidenniedrigwasser-Linie die Pigmentmenge auch

ug Pigmente /

$\mathrm{cm}^{3}$ Sediment

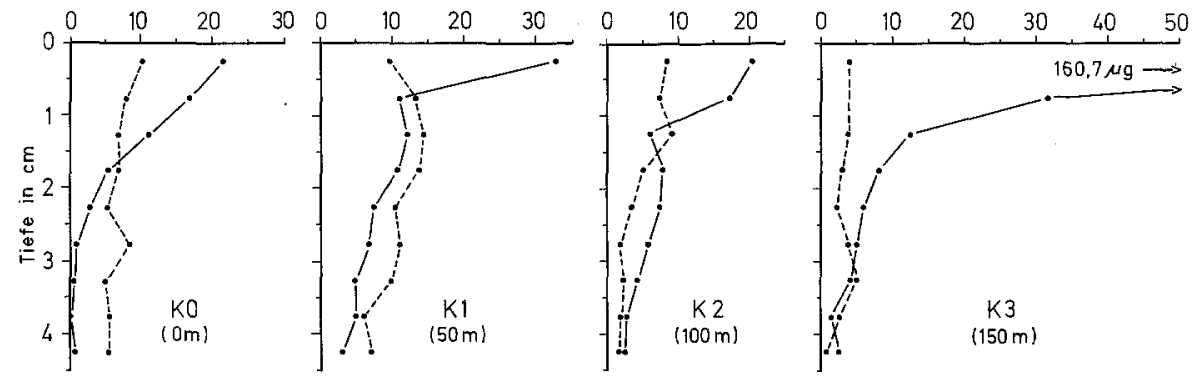

Juni 1973
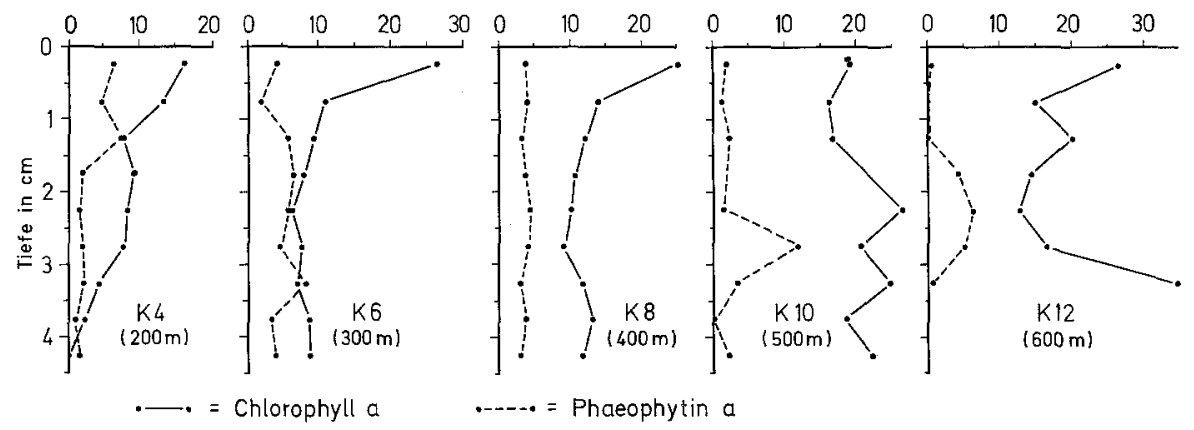

Abb. 11: Untersuchungsgebiet Kampen/Sylt. Vertikalverteilung von Chlorophyll a und Phaeophytin a im Sediment des Abwasserpriels (Werte vom Juni 1973). Unter der Stationsbezeichnung wird jeweils die Entfernung von der Einleitungsstelle angegeben

unterhalb der Oberflächenschicht teilweise deutlich an (im Juni bei den Stationen K 10 und K 12 und im September bei K 12) und erreichte höhere Werte als in der Oberflächenschicht, aber das gleiche Phänomen zeigte sich auch dicht an der Einleitungsstelle (Station K 1 im September: höchste Pigmentmenge in $2 \mathrm{~cm}$ Tiefe). Die Diffusionstiefe des Sauerstoffs (vgl. Tab. 4) - ein indirekter Einfluß des Abwassers - spielt daher ebenfalls keine Rolle für die Vertikalverteilung der lebenden Algenzellen im Sediment.

Die Vertikalverteilung der Phaeopigmente in den Zonen unterschiedlicher Abwasserbelastung war so unterschiedlich, daß kein deutlicher Trend zu erkennen war. Im Juni lag lediglich der Anteil des Phaeophytin a in bestimmten Tiefen häufiger über dem des aktiven Chlorophyll a als im September (Abb. 11 und 12). Es fällt allerdings auf, daß die Phaeopigmente fast nur im oberen Bereich des Abwasserpriels höhere Werte unter der 


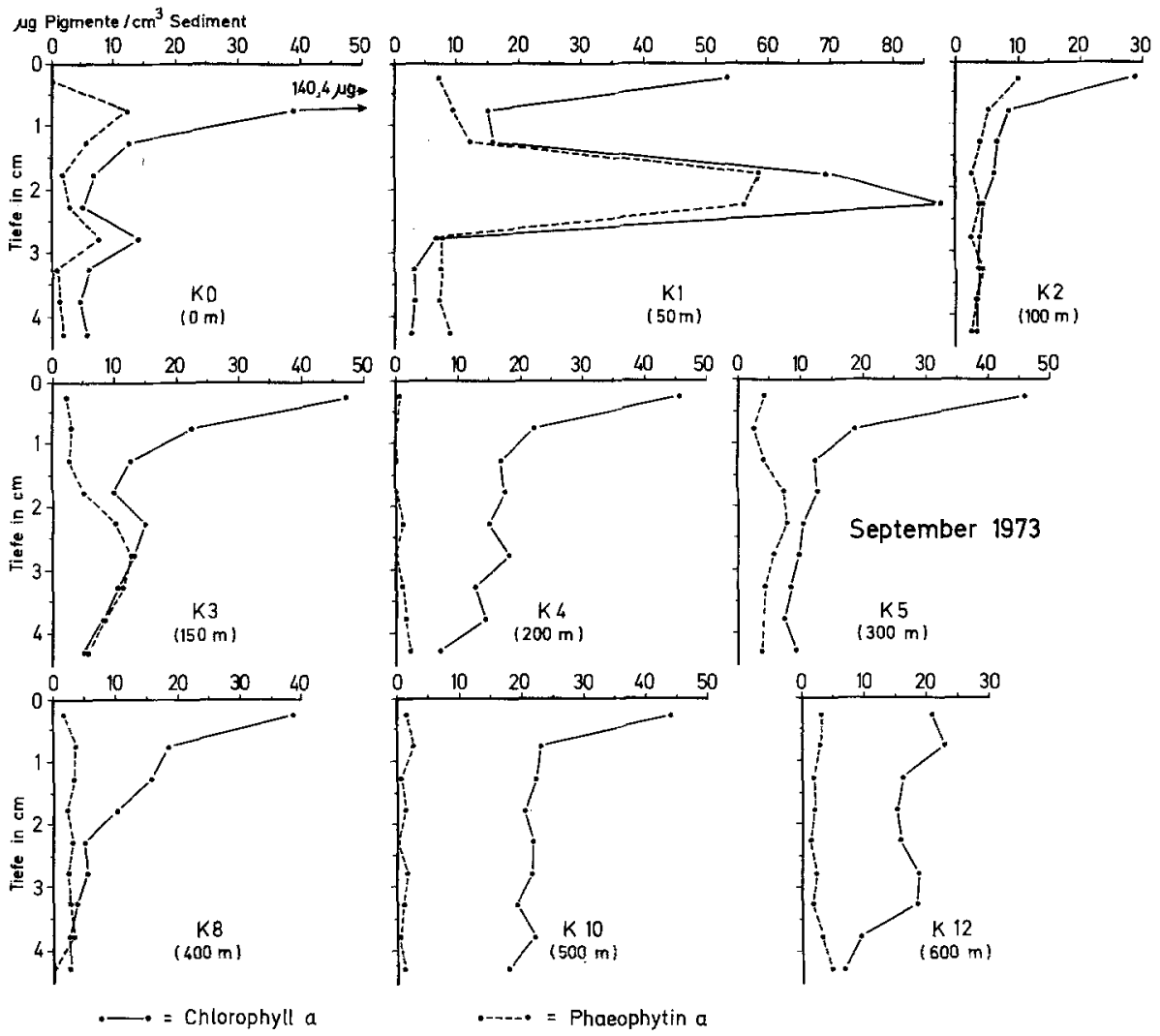

Abb. 12: Untersuchungsgebiet Kampen/Sylt. Vertikalverteilung von Chlorophyll a und Phaeophytin a im Sediment des Abwasserpriels (Werte vom September 1973). Unter der Stationsbezeichnung wird jeweils die Entfernung von der Einleitungsstelle angegeben

Sedimentoberfläche zeigten als das lebende Chlorophyll. Es ist zu vermuten, daß hier häufiger die Oberflächenalgen durch Klärschlamm überdeckt wurden und zum größten Teil abstarben. Bestätigt wird diese Interpretation dadurch, daß im Juni, kurz nach einer Umstellung im Klärwerk, nach der das eingeleitete Abwasser sehr schlammhaltig war, wesentlich häufiger höhere Werte für totes Chlorophyll gefunden wurde als im folgenden September.

\section{Einfluß des Abwassers auf die Stoffwechseldynamik der Wattensedimente}

Stoffwechseldynamische Untersuchungen wurden 1973 im Untersuchungsgebiet von Kampen auf Sylt im Juni (kurz vor Beginn der Badesaison bei noch ungenügender Abwasserklärung), zweimal im September (bei unterschiedlichen Lichtverhältnissen) und Ende November bei Lufttemperaturen nahe $0^{\circ} \mathrm{C}$ durchgeführt. 


\section{Die Primärproduktion in den Zonen unterschiedlicher Abwasserbelastung}

Wie mikroskopische Untersuchungen des Oberflächensedimentes im Abwasserpriel bei Kampen zeigten, besiedelten in erster Linie Cyanophyceen den unmittelbaren Einleitungsbereich, während sonst im gesamten Wattengebiet Diatomeen das Sediment bedeckten.

Beide Algengesellschaften reagierten in den drei Untersuchungszeiträumen deutlich auf den Abwassereinfluß (Abb. 13): Die abwasserresistenten Cyanophyceen (König, 1968)
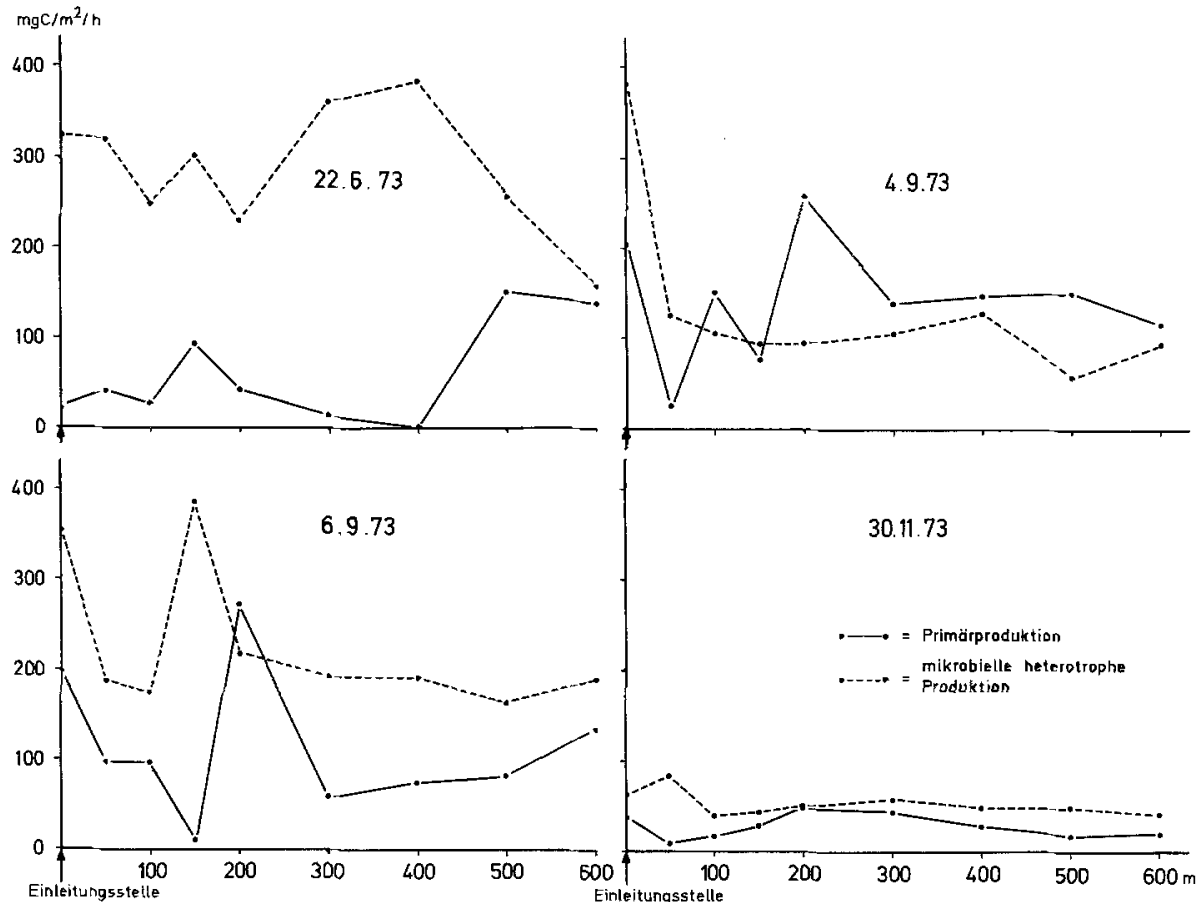

Abb. 13: Untersuchungsgebiet Kampen/Syit. Primärprodukrion und mikrobielle, heterotrophe Produktion der Sedimentoberfläche im Abwasserpriel als Kohlenstoffaufnahme pro $\mathrm{m}^{2}$ Sedimentoberfläche in einer Stunde. Lichtwerte während der Messungen: 22. 6. $1973=92000-96000$ Lux; 4. 9. $1973=20000-40000 \mathrm{Lux} ; 6.9 .1973=36000-56000 \mathrm{Lux} ; 30.11 .1973=9000-28000 \mathrm{Lux}$

zeigten jeweils ein Produktionsmaximum bei oder direkt an der Einleitungsstelle (Entfernung 0 bis $50 \mathrm{~m}$ ). Die Diatomeen wiesen erhöhte Produktionsraten in der anschließenden, schwächer belasteten Zone auf (Entfernung von der Einleitungsstelle: 150-200 m). Dabei lagen die absoluten Produktionswerte der Diatomeen jeweils deutlich über denen der Cyanophyceen. Beide Algengruppen wurden also durch das Abwasser, bzw. durch die vom $A$ bwasser in das Watt eingebrachten Mineralien, innerhalb bestimmter Zonen zu einer höheren Primärproduktion angeregt. Diese Zonen beschränkten sich auf die Gebiete, die einerseits den Algen entsprechend ihrer ökologischen Potenz noch Lebensmöglichikeiten gaben, andererseits aber auch noch hohe Abwasserkonzentrationen aufwiesen. Im Ubergangsgebiet zwischen beiden Algen-Gesellschaften lag jeweils ein Abschnitt mit sehr 
geringen Produktionsraten. Dies weist darauf hin, daß hier weder für die Cyanophyceen noch für die Diatomeen trotz des guten Nährstoffangebotes Entwicklungsmöglichkeiten bestanden.

Die saisonalen Unterschiede im durchschnittlichen Produktionsniveau spiegelten die durch den Tourismus bedingten jahreszeitlichen Schwankungen der eingeleiteten Abwassermengen wieder. Im Juni waren die Produktionsraten im oberen und mittleren Abwasserpriel, im Vergleich zu anderen Küsten- und Wattengebieten, entsprechend dem derzeitig geringen Abwasseranfall noch nicht überhöht (Grøntved, 1960; Leach, 1970). Die hohen Werte an der TNW-Linie lassen sich auf den natürlichen Beginn einer intensiven Wachstumsphase zurückführen, in der Algen eine größere Aktivität zeigen. Zur gleichen Zeit konnte beobachtet werden, daß eine Besiedlung der Watten mit Diatomeen - zu erkennen an der typischen Braunfärbung der Sedimentoberfläche - bei der Niedrigwasserlinie einsetzte. Es scheint sich bei dieser gesteigerten Produktion also in erster Linie um einen "natürlichen" Vorgang zu handeln, obwohl ein Einfluß des Abwassers nicht ausgeschlossen werden kann.

Im September lagen die Produktionsraten insgesamt höher, was auf die tourismusbedingte vermehrte Abwassermenge zurückgeführt werden muß. Ein Einfluß der Jahreszeit auf die Höhe der Primärproduktion kann ebenso ausgeschlossen werden (Cadée \& Hegemann, 1974) wie eine Beeinflussung durch unterschiedliches Lichtangebot; die Messungen im September wurden sogar bei schwächerem Licht als im Juni durchgeführt.

Die geringen Produktionsraten im Winterhalbjahr sind dagegen auf die niedrige Temperatur $\left(0,5-2,0^{\circ} \mathrm{C}\right.$ auf der Sedimentoberfläche), die ungünstigen Lichtverhältnisse (vgl. Gargas, 1970) und den geringen Abwasseranfall zurückzuführen.

Ein Vergleich der Chlorophyll a-Menge im Sediment mit den Primärproduktionsraten zeigt, daß zwar beide Komponenten durch die Abwassereinleitung erhöht wurden, jedoch keine direkte Korrelation zwischen ihnen besteht. Eine Biomassenbestimmung von Algen (durch die Analyse des Chlorophyll a) berechtigt also nicht dazu, Aussagen über die Produktion zu machen (siehe auch Cadé \& Hegeman, 1974). So konnten aus den Untersuchungen im Juni nur Korrelationskoeffizienten zwischen Biomasse und Produktion von $r=0,23$ und im September von $r=0,32$ bzw. $r=0,5$ ermittelt werden. Alle Werte sind auf dem $5 \%$-Niveau $\left(P_{0,05}=0,66\right)$ nicht abzusichern.

\section{Einfluß des Abwassers auf die beterotrophe mikrobielle Produktion}

Die heterotrophe Produktion der Destruenten erreichte im Untersuchungsgebiet von Kampen höhere Werte als die Primärproduktion (Abb. 13). Am deutlichsten erkennbar war dies im Juni. Aber auch im September (6. 9. 1973) und November lagen die Produktionswerte der heterotrophen Mikroorganismen (bezogen auf Kohlenstoffgewinn) noch eindeutig über denen der Algen, wenn auch die absoluten Werte generell abgesunken waren. Für diese gesteigerte heterotrophe Kohlenstoff-Fixierung wird als Ursache der Abwassereinfluß angesehen.

Deutlicher wird eine Beeinflussung der heterotrophen Mikroorganismen bei einem Vergleich ihrer Produktionsraten mit der graduell unterschiedlichen Abwasserbelastung zwischen Einleitungsstelle und TNW-Linie. Außer in der Vorsaison (Juni) zeigten die 


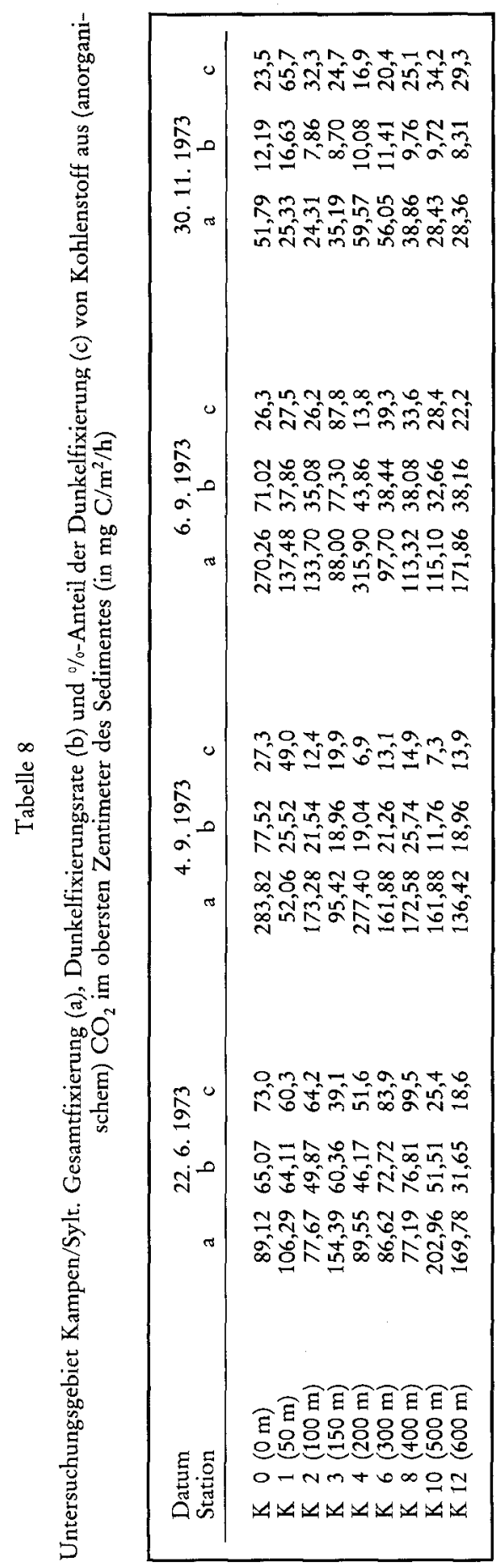


Bakterien ihre maximale Produktion im hoch belasteten oberen Prielabschnitt. In der schwächer belasteten mittleren und unteren Zone fielen dagegen die Werte parallel zur Abwasserkonzentration ab. Nur im Juni waren die höchsten Produktionswerte - wie schon bei den Autotrophen - in den Bereich mittlerer Belastung verschoben. Allerdings kann hier nicht von einem stark ausgeprägten Maximum gesprochen werden, da die Werte im gesamten Priel sehr hoch waren. Eine schlechtere Abwasserklärung in der Vorsaison und eine kurz vor der Meßperiode erfolgte Änderung der biologischen Reinigungsmethode im Klärwerk, durch die es zu einer kurzzeitig höheren Belastung des Vorfluter-Watts kam, lassen sich als mögliche Ursachen anführen.

Durch einen Vergleich der Prozentanteile für die $\mathrm{CO}_{2}$-Dunkelfixierung (berechnet als $\mathrm{mg} \mathrm{C} / \mathrm{m}^{2} / \mathrm{h}$ ) der Prielsedimente (Tab. 8) mit den Ergebnissen anderer Autoren für unbelastete Sedimente läßt sich nachweisen, daß das Abwasser die Ursache für die Verschiebung des Produktions-Respirations-Verhältnisses zugunsten der Heterotrophen war. So fand Grøntved $(1960,1962)$ in dänischen Flachwassergebieten 13,6 \% bzw. 7,2-8,9 \% für die Dunkelfixierung, in einem verschmutzten Gebiet sogar 37,7\%. Gargas (1972) gibt für die Ostsee 10-15\% und maximal $40 \%$ an. Diese Werte liegen im Schnitt niedriger als die für das Untersuchungsgebiet bei Kampen ermittelten Werte (Ausnahme: einige Werte am 4. 9. 1973); die Maxima beider Autoren bleiben sogar weit unter denen aus dem Abwasserpriel, die 49-99,5\% erreichten. Dieser hohe Anteil der Dunkelfixierung an der Gesamtfixierungsrate im Abwassereinleitungsgebiet bei Kampen muß daher auf den gesteigerten Import abbaubaren organischen Materials aus dem Abwasser zurückgeführt werden.

\section{Beziehungen zwischen den Komponenten der Bioaktivität und einigen Sedimentfaktoren}

Aus den Messungen der Primärproduktion und der mikrobiellen heterotrophen Produktion sowie den Chlorophyll-Analysen im Sediment sind folgende Zusammenhänge ersichtlich: (1) Die Gesamtbioaktivität wird gegenüber unbelasteten Wattenflächen durch eine künstliche Trophie- und Saprobitätserhöhung gesteigert. (2) Der Anteil der bakteriellen Produktion tritt gegenüber der Primärproduktion in den Vordergrund und nimmt dadurch die führende Rolle beim Nahrungsangebot für die auf ihr basierenden Nahrungsketten - besonders für Detritusfresser - im Watt ein. (3) Die Biomasse der Primärproduzenten (und vermutlich auch der heterotrophen Mikroorganismen) im Sediment wird durch das Abwasser angehoben.

Dabei ist jedoch die Primärproduktion nicht mit der Biomasse der Algen korreliert. Vielmehr bestehen eindeutige Beziehungen zwischen dem Gehalt des Sedimentes an funktionellem Chlorophyll a und der bakteriellen Produktion: Korrelationskoeffizienten $r$ $=0,92$ (Juni) und $r=0,93$ (September); Signifikanzschwelle für $P_{0,05}=0,666$ (Tab. 9). Beide Komponenten sind wiederum mit dem Anteil der Silt-Ton-Fraktion sowie der organischen Substanz im Sediment hoch korreliert (Tab. 9).

$\mathrm{Daß}$ die Primärproduktion insgesamt keine Korrelation mit den Sedimentfaktoren oder dem Grad der Abwasserbelastung aufweist, ist teilweise auf das getrennte Vorkommen der Cyanophyceen- und Diatomeen-Gesellschaften zurückzuführen. Beide Gruppen zeigten jedoch innerhalb ihrer Verbreitung im Priel eine Abhängigkeit von der Abwasser- 
belastung: Am Beginn ihres Siedlungsbereiches, wo der relativ höchste Abwassereinfluß besteht, wiesen sie jeweils die intensivste Produktion und höchste Biomasse auf.

Kein statistischer Zusammenhang besteht zwischen der Produktion der autotrophen und der der heterotrophen Mikroorganismen. Dies scheint ebenfalls durch die Verteilung der Algengesellschaften begründet zu sein.

Die vorliegenden Ergebnisse zeigen, daß im abwasserbelasteten Wattengebiet bei der Produktion lebender Biomasse den Destruenten im Vergleich zu den Primärproduzenten die größere Bedeutung zukommt. Dies hat vornehmlich zwei Ursachen: (1) Wegen der

Tabelle 9

Untersuchungsgebiet Kampen (Sylt). Korrelationen zwischen den Komponenten der Bioaktivität und den Sedimentfaktoren (September 1973) $r_{p 0,05}=0,666.1=$ Algenbiomasse (Chlorophyll a), $2=$ Phaeophytin a, $3=$ Primärproduktion, $4=$ Heterotrophe, mikrobielle Produktion, $5=\log$ des P/R-Quotienten, 6 = Sedimentmedian, $7=$ Silt-Ton-Fraktion, $8=$ Anteil der organischen Substanz

\begin{tabular}{|c|c|c|c|c|c|c|c|c|}
\hline & 1 & 2 & 3 & 4 & 5 & 6 & 7 & 8 \\
\hline 1 & - & 0,19 & 0,332 & 0,929 & $-0,352$ & 0,764 & 0,743 & 0,844 \\
\hline 2 & & - & $-0,451$ & 0,387 & $-0,731$ & 0,239 & 0,537 & 0,575 \\
\hline 3 & & & - & 0,29 & 0,651 & 0,308 & $-0,044$ & $-0,071$ \\
\hline 4 & & & & - & $-0,446$ & 0,723 & 0,832 & 0,884 \\
\hline 5 & & & & & - & $-0,257$ & $-0,581$ & $-0,729$ \\
\hline 6 & & & & & & - & 0,315 & 0,562 \\
\hline 7 & & & & & & & - & 0,905 \\
\hline 8 & & & & & & & & - \\
\hline
\end{tabular}

geringen Eindringtiefe des Lichtes in das Sediment steht den Algen für die Photosynthese praktisch nur die Sedimentoberfläche zur Verfügung, während die aeroben Destruenten die gesamte oxydierte Sedimentschicht nutzen können. (2) Durch das Sedimentieren der feinen, partikulären Substanzen aus dem Abwasser, zusammen mit dem vom Meer herangeführten Detritus, stehen den Bakterien große Oberflächen für eine Besiedlung zur Verfügung, die kontinuierlich von der "Nährlösung Abwasser" umspült werden.

"Grazing"-Effekte der Fauna wurden bei diesen Betrachtungen unberücksichtigt gelassen, da keine statistischen Zusammenhänge mit den Komponenten der Bioaktivität festgestellt werden konnten.

\section{Auswirkungen des Abwassers auf das Makrozoobenthos}

Der Wasserkörper im Gezeitenbereich wird ständig versetzt, durchmischt und ausgetauscht. Er ist daher wenig geeignet, um Auswirkungen von lokalen Verschmutzungsquellen auf die Organismen zu quantifizieren. Dies gilt insbesondere für die vagilen Faunenelemente, während das sessile und hemisessile Makrobenthos im Einleitungsbereich von Abwässern den veränderten chemischen, physikalischen und biologischen Umweltbedingungen der unmittelbaren Umgebung dauernd ausgesetzt ist. Es reflektiert also die Bedingungen ihrer unmittelbaren Umgebung integrierend über einen längeren Zeitab- 
schnitt vor der Probennahme (SCCWRP, 1973). Aus diesem Grund werden BenthosGesellschaften häufig als Indikatoren für die lokale Umweltsituation herangezogen (Reish, 1955, 1959, 1960, 1972; Oglesby \& Jamison, 1968; Tulkki, 1968; Bagge, 1969; Jarvekülg, 1970; Perkins \& Abbott, 1972; Leppäkosky, 1975). Für die vorliegenden MakrobenthosAnalysen wurden bei Kampen im Sommer 1970 vor Inbetriebnahme der neuen Belebtschlammanlage im Klärwerk, als das eingeleitete Abwasser noch ungenügend geklärt wurde (Otte, 1977), und 1972/73 in Kampen, List und Braderup quantitative Untersuchungen durchgeführt, die im Sommerhalbjahr 1974 durch Paralleluntersuchungen bei Vancouver/Canada ergänzt wurden.

\section{Die Artenvielfalt in den abwasserbeeinflußten Wattengebieten}

Die Artenvielfalt in den abwasserbeeinflußten Watten wurde im Vergleich zu anderen Wattengebieten im allgemeinen nicht negativ durch die Einleitungen beeinflußt. In einzelnen stark belasteten Zonen kam es trotzdem lokal zur drastischen Reduktion des Artenspektrums.

In den drei Sylter Untersuchungsgebieten wurden insgesamt 32 Makrobenthos-Arten in den quantitativen Proben gefunden. Die gleiche Anzahl (32 Arten) ergaben die Probenauswertungen für das Wattengebiet bei Vancouver (Tab. 10 und 11). Es dominierten jeweils die Polychaeten mit 16 bzw. 17 Arten. Während jedoch bei Sylt die Mollusken (8 Arten) artenreicher vertreten waren als die Crustaceen (5 Arten), wiesen im Gebiet bei Vancouver die Crustaceen (11 Arten) eine größere Vielfalt als die Mollusken (3 Arten) auf (nicht weiter artlich differenziert und daher nur als Gruppe angegeben wurden die Oligochaeten und Nemertinen).

In den Einleitungsbereichen bei Sylt kamen folgende Arten gleichzeitig in allen Gebieten vor: Hydrobia ulvae, Macoma balthica, Nereis diversicolor, Eteone longa, Pygospio elegans, Capitella capitata, Arenicola marina, Corophium volutator sowie Oligochaeten und Nemertinen (Tab. 10).

Hinzugerechnet werden muß noch Mya arenaria. Diese Muschel wurde im Untersuchungsgebiet bei List nur qualitativ festgestellt, da sie hier so tief im Sediment siedelte, daß sie von dem für die quantitativen Analysen benutzten Sediment-Stechrohr nicht erfaßt wurden.

Diese Arten können als Charakter-Arten für die belasteten Gebiete angesehen werden, zumal sie auch (bis auf die Nemertini) die höchsten Abundanzen aufwiesen.

Diese relativ große Artenvielfalt der jeweiligen Untersuchungsgebiete war innerhalb der einzelnen Transekte (Kampen/Sylt und Vancouver/Canada) bei weitem geringer. Die Artenzahlen schwankten hier zwischen 11 und 22 pro Transekt (Tab. 10 und 11). Diese reduzierten Artenzahlen berechtigen jedoch nicht dazu, von einer Dezimierung des Artenspektrums in abwasserbelasteten Watten zu sprechen, da Beukema (1976) in vergleichbaren, aber unbelasteten Transekten im holländischen Wattenmeer ähnliche Werte für die Artenvielfalt fand. Allerdings berücksichtigen solche summarischen Angaben für das gesamte Untersuchungsgebiet oder für einzelne Transekte keine lokal eingeschränkten Zonierungen und Degradationsareale. 
Tabelle 10

Vorkommen der in den quantitativen Benthosproben gefundenen Arten in den Untersuchungsgebieten auf Sylt

\begin{tabular}{|c|c|c|c|c|c|c|c|}
\hline \multirow{2}{*}{ Species } & & \multicolumn{2}{|c|}{ Kampen 1970} & \multicolumn{2}{|c|}{ Kampen $1972 / 3$} & \multirow{2}{*}{ List } & \multirow{2}{*}{$\begin{array}{l}\text { Brade- } \\
\text { rup }\end{array}$} \\
\hline & & Priel & Watt & Priel & Watt & & \\
\hline \multicolumn{8}{|l|}{$\begin{array}{l}\text { Poly ch a e } t \text { a: } \\
\text { Lepidonotus squamatus }\end{array}$} \\
\hline Nereis diversicolor & & + & + & + & + & + & + \\
\hline Eteone longa & & & + & + & + & + & + \\
\hline Anaitides groenlandica & & + & & & & t & \\
\hline $\begin{array}{l}\text { Anattzdes mucosa } \\
\text { Mysta spec. }\end{array}$ & & & + & & & + & \\
\hline Scoloplos armiger & & + & & + & & & \\
\hline Microspio wireni & & & + & & & & \\
\hline Polydora ligni & & + & + & & & & \\
\hline Spio filicornis & & + & + & & & & \\
\hline Pygospio elegans & & + & $\begin{array}{l}+ \\
+\end{array}$ & + & + & + & + \\
\hline $\begin{array}{l}\text { Scolecolepis fultginosa } \\
\text { Tharyx marioni }\end{array}$ & & + & $\begin{array}{l}+ \\
+\end{array}$ & + & & & + \\
\hline Capitella capitata & & + & + & + & + & + & + \\
\hline $\begin{array}{l}\text { Notomastus latericus } \\
\text { Heteromastus filiformis }\end{array}$ & & + & & + & & + & \\
\hline Arenicola marina & & + & & + & + & + & + \\
\hline $\begin{array}{l}\text { Mollusca: } \\
\text { Hydrobia ulvae }\end{array}$ & & + & + & + & + & + & + \\
\hline Littorina littorea & & + & + & & & + & \\
\hline Lepidochiton cinereus & & & + & & & & \\
\hline Macoma balthica & & + & + & t & + & + & + \\
\hline Mya arenaria & & + & & + & + & & + \\
\hline $\begin{array}{l}\text { Cardium lamarki } \\
\text { Cardium edule }\end{array}$ & & + & & + & & & \\
\hline Mytilus edulis & & + & + & & & & \\
\hline $\begin{array}{l}\text { Crust a ce a : } \\
\text { Diastylis ratbkei }\end{array}$ & & & & & & + & \\
\hline Corophium volutator & & + & + & + & + & + & + \\
\hline Gammarus locusta & & + & & & & & \\
\hline Carcinus maenas & & & + & + & & + & \\
\hline Crangon crangon & & + & & & & & \\
\hline Oligochaeta: & & + & + & + & + & + & + \\
\hline Nemertini: & & & & + & + & + & + \\
\hline Gesamtartenzahlen: & 32 & 20 & 19 & 17 & 11 & 15 & 12 \\
\hline
\end{tabular}

\section{Verteilung des Makrobenthos in Abhängigkeit vom Verschmutzungsgradienten}

Eine Einleitung von kommunalem Abwasser in Wattengebiete führt zum Aufbau eines Verschmutzungsgradienten im Vorfluter, d. h. die hohe Belastung am Ablauf nimmt mit zunehmender Entfernung ab. Auf diese graduell abnehmende Belastung reagiert das Makrobenthos im Abwasserpriel bzw. Kanal grundsätzlich anders als auf den trockenfallenden Wattflächen. Beide Biotope sollen daher getrennt betrachtet werden. 


\section{Tabelle 11}

Vorkommen der Arten aus den quantitativen Benthosproben in den verschiedenen Transekten im Untersuchungsgebiet Vancouver

\begin{tabular}{|c|c|c|c|c|c|c|c|c|}
\hline Species & & & anse & & & alle & nur & nur \\
\hline \multirow{2}{*}{\multicolumn{9}{|c|}{ Polychaeta: }} \\
\hline $\begin{array}{l}\text { Eteone longa } \\
\text { Eta: }\end{array}$ & & & & & & & & \\
\hline Nereis limnicola & + & + & + & & & & + & \\
\hline Glycinde picta & & & & & + & & & + \\
\hline Glyceridae spec. & & & & + & & & + & \\
\hline Nephtys spec. & & & & & + & & & + \\
\hline Scoloplos armiger & & & & + & & & t & \\
\hline Capitella capitata & + & + & & & + & & & \\
\hline Barantolla americana & & + & + & + & + & & & \\
\hline $\begin{array}{l}\text { Polydora kempi japonica } \\
\text { Polydora ligni }\end{array}$ & + & + & + & + & $\begin{array}{l}+ \\
+\end{array}$ & + & & + \\
\hline Pygospio elegans & + & + & + & + & & & + & \\
\hline Paraonis platybranchia & & & & + & + & & & \\
\hline Manayunkia aestuarina & + & + & & & & & + & \\
\hline Amphicteis spec. I & + & + & + & & + & & & \\
\hline $\begin{array}{l}\text { Armandia brevis } \\
\text { Euzonus williamsi }\end{array}$ & & & + & & + & & + & + \\
\hline \multirow{2}{*}{\multicolumn{9}{|c|}{$\begin{array}{l}\text { Mollu u c a : } \\
\text { Macoma inconspicua }\end{array}$}} \\
\hline & & & & & & & & \\
\hline $\begin{array}{l}\text { Mya arenaria } \\
\text { Clinocardium nuttalli }\end{array}$ & + & + & + & $\begin{array}{l}+ \\
+\end{array}$ & $\begin{array}{l}+ \\
+\end{array}$ & + & & \\
\hline \multicolumn{9}{|l|}{ Crustacea: } \\
\hline $\begin{array}{l}\text { Coropbium salmonis } \\
\text { Anisogammarus confervicolus }\end{array}$ & 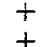 & $\begin{array}{l}t \\
+\end{array}$ & + & & + & & + & \\
\hline $\begin{array}{l}\text { Anusogammarus confervicolus } \\
\text { Eohaustorius washingtonianus }\end{array}$ & + & + & + & + & & & $\begin{array}{l}+ \\
+\end{array}$ & \\
\hline Paraphoxus spec. & + & & + & + & + & & + & \\
\hline $\begin{array}{l}\text { Tanais spec. } \\
\text { Cumella spec. }\end{array}$ & & & & & + & & & + \\
\hline Lamprops spec. & & & & + & + & & & \\
\hline Archaeomysis grebnitzkii & & & & & + & & & + \\
\hline Acantbomysis macropsis & & & & & + & & & + \\
\hline Cancer magister & & & & & + & & & + \\
\hline Crangon franciscorum & & & + & & & & + & \\
\hline Oligochaeta: & + & + & + & & + & & & \\
\hline Nemertini: & + & + & + & + & + & + & & \\
\hline Gesamtartenzahlen: & 14 & 14 & 16 & 14 & 22 & 5 & 10 & 8 \\
\hline
\end{tabular}

Sowohl im Abwasserpriel des Untersuchungsgebietes Kampen als auch im Abwasserkanal bei Vancouver fand sich an der Einleitungsstelle eine Degradationszone. Die Artenvielfalt und die Individuenabundanz waren hier drastisch herabgesetzt oder das Makrobenthos fehlte völlig (Abb. 14 und 15). Die Ausdehnung dieser Zone hing von der Menge und Qualität des eingeleiteten Abwassers ab: Während 1970 im Priel bei Kampen erst im Abstand von 50 Metern eine Besiedlung des Sedimentes begann, war 1973 - bei etwa gleicher Abwassermenge, aber wesentlich besser geklärtem Abwasser - keine deutliche Degradationszone mehr zu finden. Arten- und Individuenzahlen waren lediglich gegenüber den nachfolgenden Stationen herabgesetzt. Im Kanal bei Vancouver dagegen 


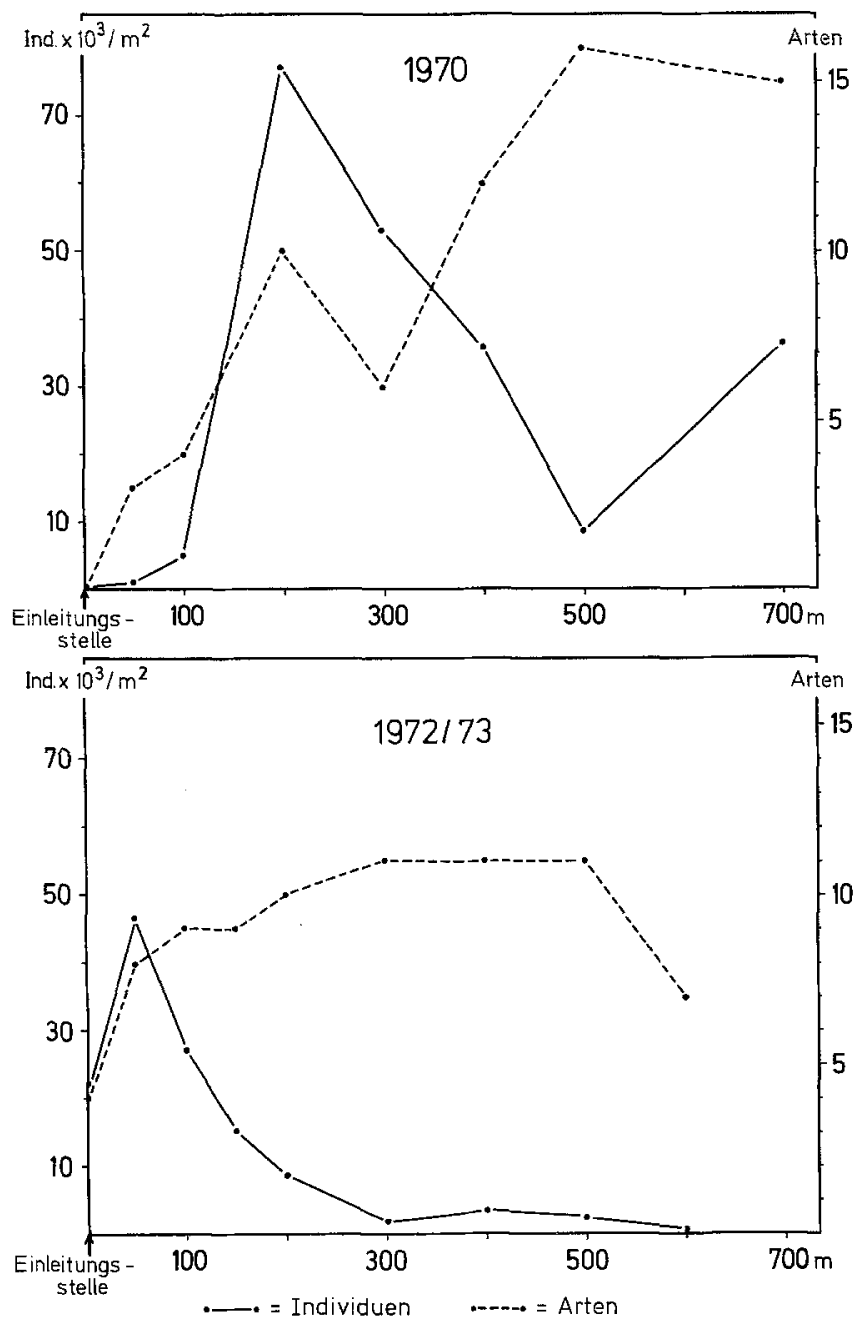

Abb. 14: Untersuchungsgebiet Kampen/Sylt. Arten- und Individuenzahlen des Makrobenthos in den Zonen unterschiedlicher Belastung im Abwasserpriel (K-Transekt)

bewirkte die große Menge des eingeleiteten Abwassers, daß erst nach rund 1500 Metern das erste Makrobenthos im Sediment zu finden war. Im Anschluß an die Degradationszone stiegen Arten- und Individuenzahlen stark an, und die Siedlungsdichte des Makrobenthos erreichte hier in der Regel ihr Maximum. Mit weiterer Entfernung vom Verschmutzungszentrum fiel die Individuenabundanz wieder ab und pendelte sich auf Werte ein, die für das Watt ohne eine zusätzliche Abwasserbelastung als "normal" gelten können; sie stellen daher Basis-Werte dar (Ludwig \& Storrs, 1970). Die Artenvielfalt stieg in diesem Bereich jedoch weiter an, wodurch die Diversität der Makrobenthos-Assoziationen zunahm.

Ebenso wie im Priel (bzw. Kanal) war auf den trockenfallenden Wattflächen im Verschmutzungszentrum (d. i. das Gebiet stärkster Verschmutzung innerhalb des Tran- 


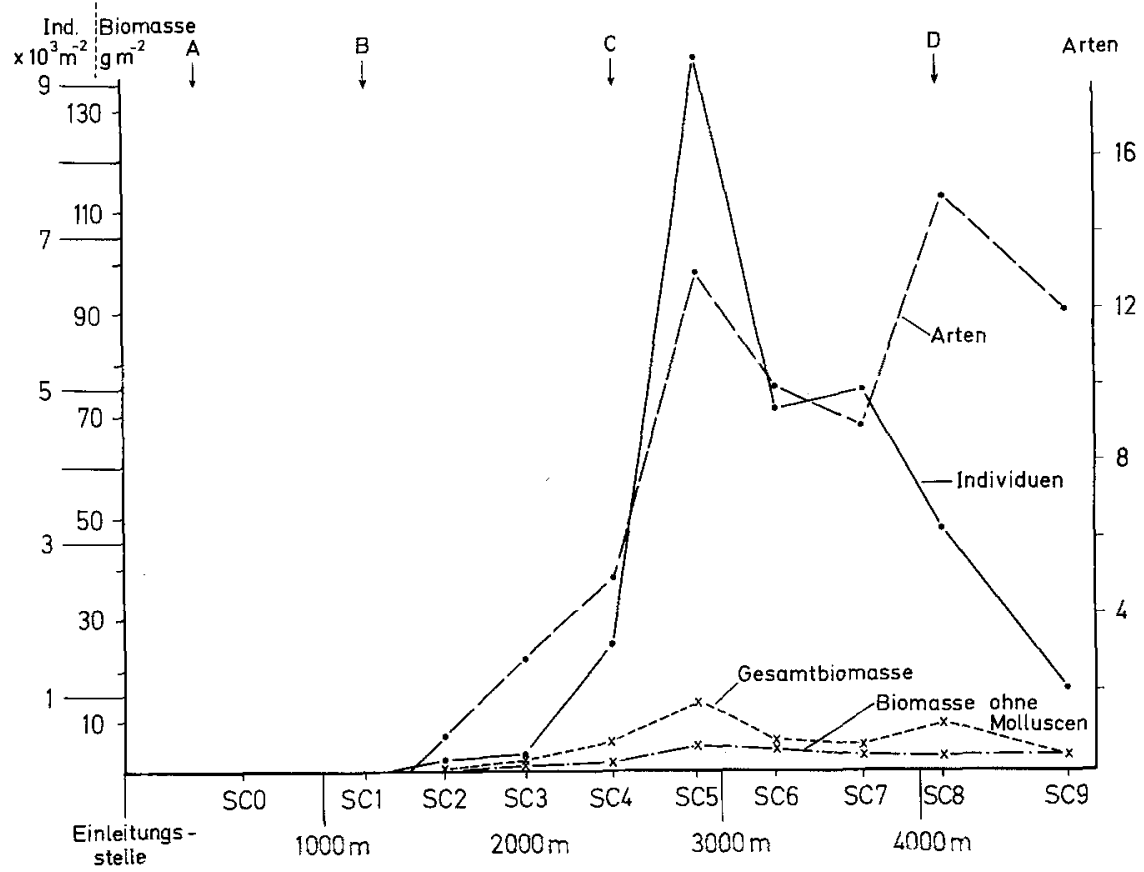

Abb. 15: Untersuchungsgebiet Vancouver. Verteilung des Makrobenthos in den Zonen unterschiedlicher Belastung im Abwasserkanal. (Arten- und Individuenzahlen sowie Biomasse an den Stationen des SC-Transektes)

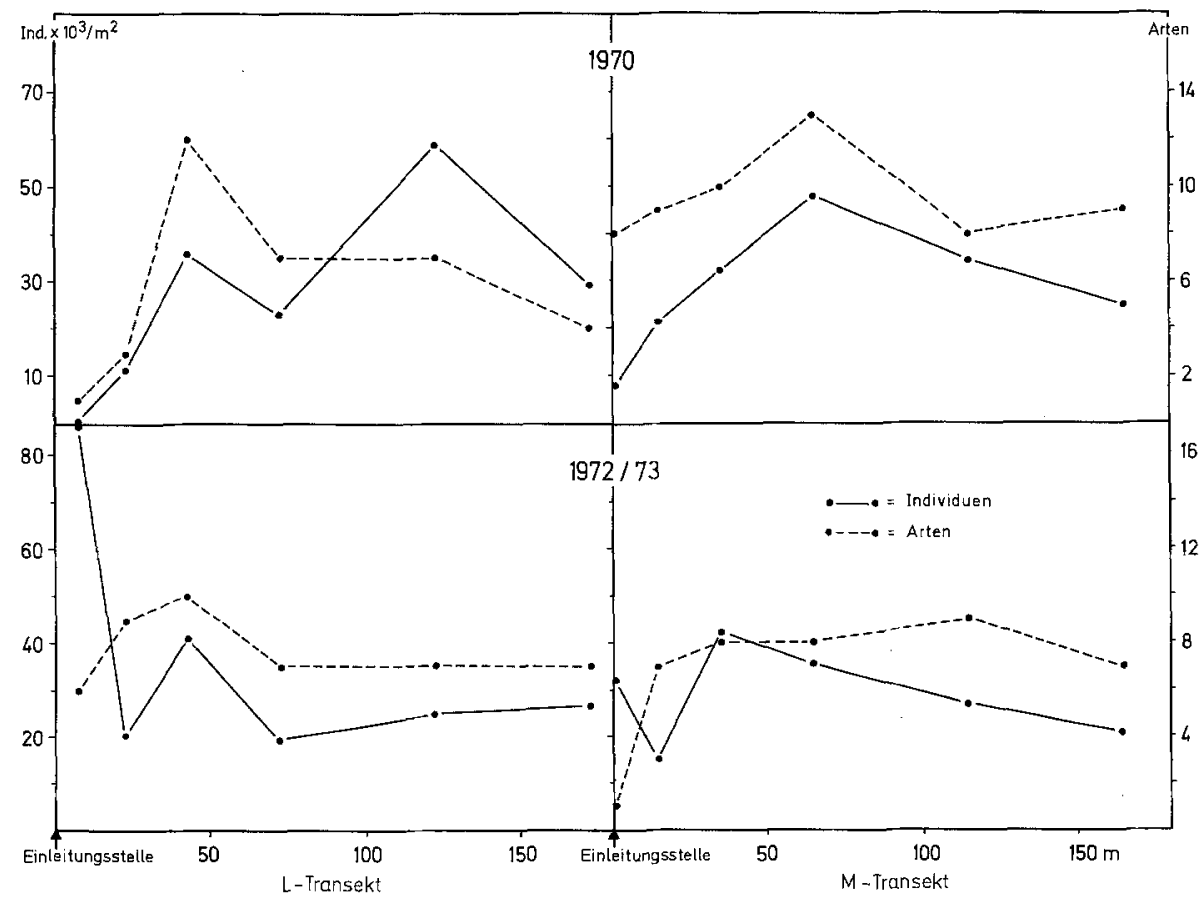

Abb. 16: Untersuchungsgebiet Kampen/Sylt. Arten- und Individuenzahlen des Makrobenthos in den Zonen unterschiedlicher Abwasserbelastung auf den trockenfallenden Wattflächen ( $\mathrm{L}$ - und $\mathrm{M}$ Transekt) 


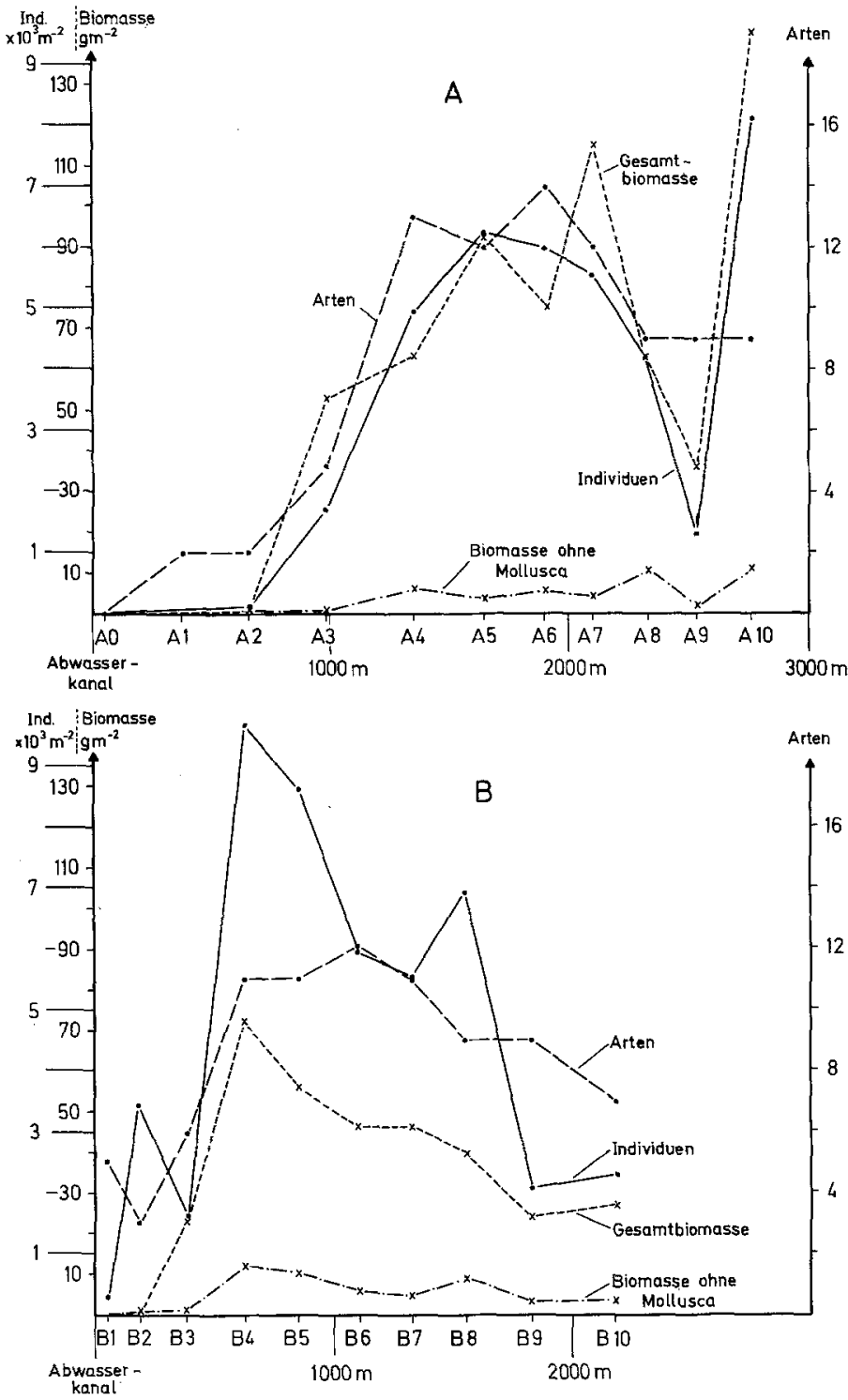

Abb. 17: Untersuchungsgebiet Vancouver. Verteilung des Makrobenthos in den Zonen unterschiedlicher Abwasserbelastung auf den Wattflächen (Transekt A und B)

sektes) eine Degradationszone festzustellen, in der entweder kein Makrobenthos existieren konnte oder doch zumindest das Artenspektrum und die Individuendichte stark vermindert waren (Abb. 16 und 17). Mit geringer werdender Belastung nahmen die Individuenund Artenzahlen auch hier sehr schnell zu. Im Gegensatz zum Abwasserpriel (oder Kanal) erreichten in dieser Zone aber nicht nur die Individuenabundanzen, sondern auch die Artenvielfalt ihr Maximum; beide Größen fielen dann in bestimmter Entfernung - je nach 


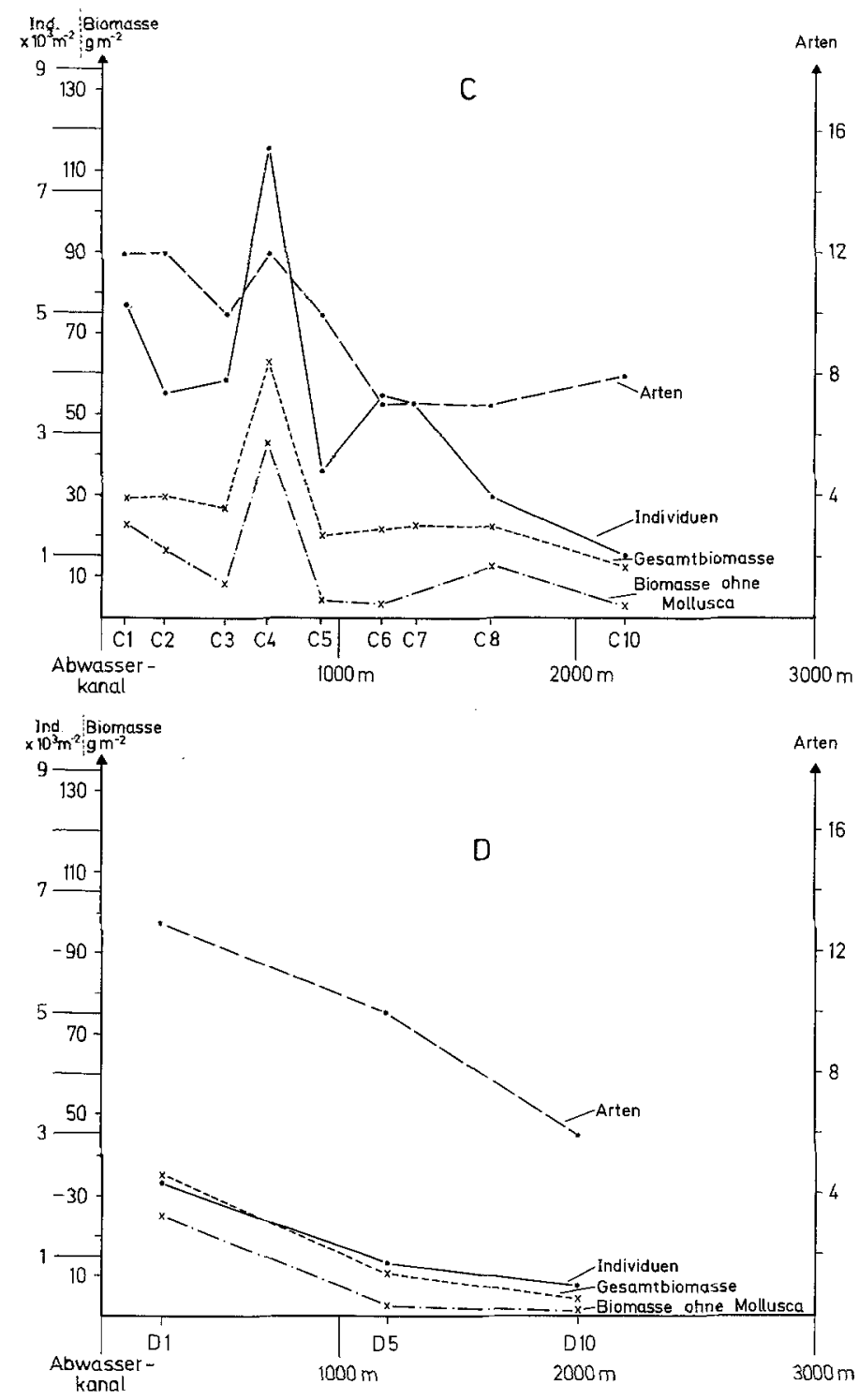

Abb. 17b: Vgl. Legende zu Abb. 17a (Trausekt C und D)

Stärke des Abwassereinflusses - auf Basis-Werte ab. Arten- und Individuenzahlen verliefen auf den Wattflächen also parallel.

Die hohen Individuenzahlen 1972 im Verschmutzungszentrum des L-Transektes werden durch ein außergewöhnliches Massenvorkommen von Hydrobia ulvae verursacht; ohne dieses Maximum entspricht die Verteilung der Abundanzen dem oben erwähnten Schema.

Die Entfernung des Arten- und Individuenmaximums vom Verschmutzungszentrum 
wird auf den Wattflächen bestimmt durch den Grad der Belastung in der Zone der höchsten Abwasserkonzentration innerhalb eines Transektes und dem Verhältnis, mit der der Abwasserbelastungsfaktor bei fortschreitender Entfernung abnimmt. Je geringer die Belastung und der Belastungsfaktor, desto mehr verschiebt sich das Maximum zum Verschmutzungszentrum. Dadurch kann es zum völligen Verschwinden einer Degradationszone kommen. Die Verteilung des Makrobenthos im Watt bei Vancouver demonstriert dies (Abb. 17): Innerhalb des A-Transektes ist noch deutlich die ausgedehnte Degradationszone zum Abwasserkanal hin zu sehen; die Arten- und Individuenzahlen erreichten erst nach etwa $1700 \mathrm{~m}$ ihr Maximum. Im B- und C-Transekt trat fast keine Verödungszone mehr auf; Arten- und Individuenmaxima lagen nur noch 800 bis $1000 \mathrm{~m}$ vom Verschmutzungszentrum entfernt. Im D-Transekt befanden sich die Maxima sogar innerhalb der am stärksten belasteten Zone. Das Abwasser im Kanal war hier bereits weitgehend mit Seewasser vermischt (Abb, 5), wodurch sich nur noch eine geringfügige Belastung und niedrige Belastungsfaktoren für die benachbarten Wattflächen ergaben.

\section{Abwasserbedingte Anderungen der Diversität innerbalb der Benthos-Assoziationen}

Die Shannon-Wiener-Funktion der Informationstheorie

$$
\left.\mathrm{H}^{*}=-\Sigma \mathrm{p}_{\mathrm{i}} \log _{2} \mathrm{p}_{\mathrm{i}}^{*}\right)
$$

(Shannon \& Weaver, 1949, 1963; McArthur \& McArthur, 1961), welche die mittlere Information pro Individuum einer Probe angibt, wurde hier als Diversitätsindex benutzt,

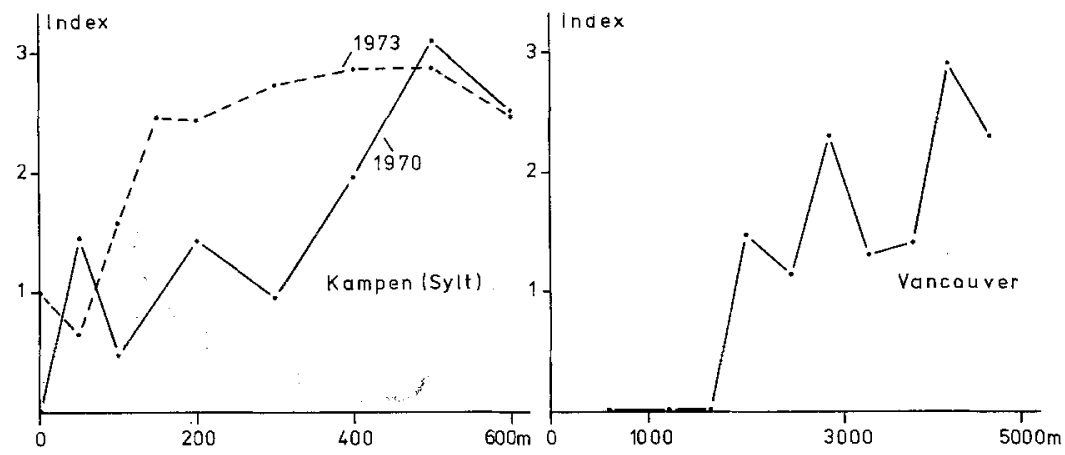

Abb. 18: Diversität der Makrofauna im Abwasserpriel (Kampen) und im Abwasserkanal (Vancouver) in Relation zum Verschmutzungsgradienten

da H' sich als weitgehend unabhängig vom Probenvolumen erwies (Sanders, 1968; Jones \& Sanders, 1972). Diese Funktion ist heute der am häufigsten benutzte und brauchbarste Diversitätsindex (Oglesby \& Jamison, 1968; Anger, 1975) zur Charakterisierung der biotischen Mannigfaltigkeit eines Okosystems (Stugren, 1972).

Ein solcher Index läßt sich zur Abschätzung des Abwassereinflusses auf die biotische Mannigfaltigkeit von Makrobenthos-Assoziationen sowie zum Vergleich belasteter und unbelasteter Watten heranziehen, da die Diversität eines Biotopes durch Abwasser beein-

\footnotetext{
${ }^{*} \mathrm{p}_{\mathrm{i}}$ ist hier der relative Anteil der $\mathrm{i}$-ten Art an der Individuenzahl pro $\mathrm{m}^{2}$ einer Station.
} 
flußt wird (Odum, 1975) bzw. negativ mit einem Umweltstreß korreliert ist (Jacobs, 1975).

Wie erwartet, zeigen die Diversitätsindizes deutlich eine Beeinflussung der biotischen Mannigfaltigkeit des Makrobenthos durch das Abwasser: In den Zonen starker Verschmutzung wies das Benthos nur eine sehr geringe Diversität auf; der Index erreichte teilweise sogar den Wert Null (Ausnahme: C- und D-Transekt bei Vancouver): Abb. 18 und 19.

Die unterschiedliche Reaktion des Makrobenthos im Abwasserpriel und auf den Wattflächen gegenüber organischer Verschmutzung wird durch die verschiedenartige Beeinflussung der Diversität in beiden Teilbiotopen noch unterstrichen: Im Abwasserkanal (Vancouver) und im Abwasserpriel (Kampen) stieg die Diversität von der Einleitungsstelle

Kampen (Sylt)
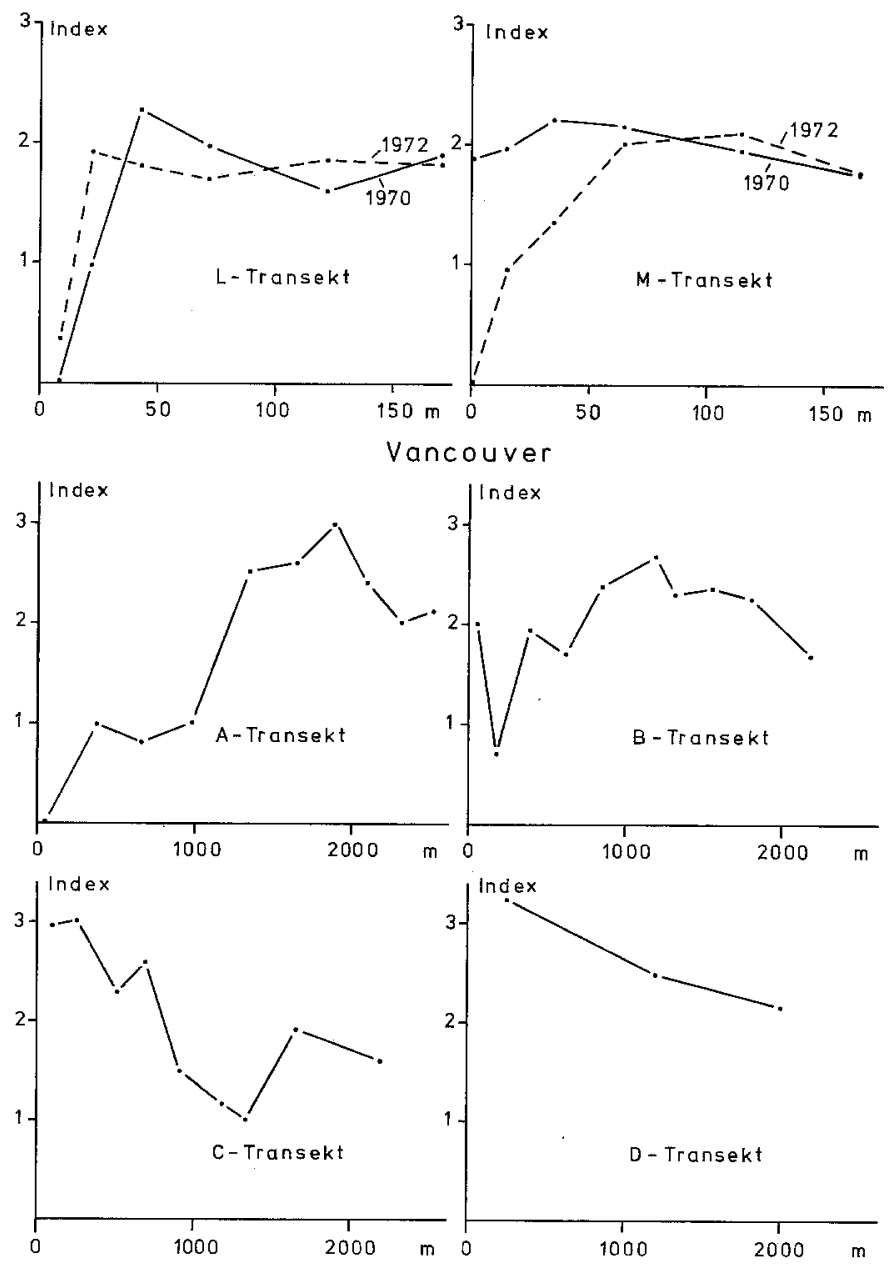

Abb. 19: Diversität der Makrofauna auf den Wattflächen (Kampen und Vancouver) in Relation zum Verschmutzungsgradienten 
innerhalb einer natürlichen Schwankungsbreite kontinuierlich zur Niedrigwasserlinie an. Diese stetige Zunahme der biotischen Mannigfaltigkeit verlief parallel zur Abnahme extremer Umweltverhältnisse, die aus der Verdünnung und Vermischung des Abwassers mit Seewasser resultierte. Auf den Wattflächen dagegen wurden in den Zonen starker und mittlerer Verschmutzung schon nach relativ geringer Entfernung vom Abwasserkanal (Transekt A und B bei Vancouver, Transekt $L$ und $M$ bei Kampen) hohe Indexwerte registriert, die im mittleren Abschnitt der jeweiligen Transekte ihr Maximum aufwiesen und dann, wenn kein Abwassereinfluß mehr nachweisbar war, wieder geringfügig absanken. Es schloß sich hier also an eine kurze Zone verminderter Diversität, die mit der Degradationszone zusammenfiel, ein Bereich an, in dem die biotische Mannigfaltigkeit - in Kombination mit maximalen Besiedlungsdichten - höher lag, als sie für diese Wattflächen ohne anthropogenen Einfluß zu erwarten wäre. Anschließend an diesen Bereich verminderte sich die Diversität dann auf Werte, die als typisch für das jeweilige Wattengebiet angesehen werden können.

Bei nur noch geringem Abwassereinfluß im Verschmutzungszentrum (Vancouver: Transekt $\mathrm{C}$ und D) wurde dann die Diversität des Makrobenthos zum Abwasserkanal hin nicht mehr herabgesetzt. Die größte biotische Mannigfaltigkeit zeigte das Benthos in diesen Transekten daher in unmittelbarer Nähe des Abwasserkanals (Abb. 19).

Eine unterschiedliche Reaktion des "Watt-Benthos" gegenüber dem "Priel-Benthos" auf eine Abwassereinleitung wird ferner durch eine Berechnung der Korrelation zwischen Abwasserbelastung (als Entfernung der Probenstationen vom Verschmutzungszentrum) und die Diversitätsindizes deutlich: Im Abwasserpriel bei Kampen waren beide Faktoren, sowohl 1970 als auch 1973, signifikant miteinander korreliert (Tab. 12), wobei der Koeffizient für die Werte von 1970 deutlich höher ausfiel - Ausdruck der damaligen stärkeren Verschmutzung durch ungenügend geklärtes Abwasser.

Tabelle 12

Untersuchungsgebiet Kampen/Sylt. Beziehungen zwischen der Diversität des Makrobenthos und der Abwasserbelastung (gemessen als Entfernung von der Einleitungsstelle)

\begin{tabular}{|llrccc|}
\hline Regionen & Transsekte & 1970 & $1972 / 73$ & $P_{0,05}$-Werte & Signifikanz \\
\hline Abwasserpriel & K-Transekt & 0,82 & 0,76 & 0,666 & + \\
Wattflächen & L-Transekt & 0,55 & 0,48 & 0,81 & - \\
& M-Transekt & $-0,43$ & 0,73 & 0,81 & - \\
\hline
\end{tabular}

Für die beiden Watten-Transekte im Untersuchungsgebiet Kampen (L- und MTransekt) war dagegen für die Daten von 1970 und 1972 keine signifikante Korrelation zwischen Diversität und Verschmutzungsstärke auf dem $5 \%$-Niveau nachzuweisen.

Diese Ergebnisse zeigen, daß die Prielfauna auf die Verschmutzung in der Form reagiert, daß die biotische Mannigfaltigkeit - ausgedrückt durch den Diversitätsindex von Shannon \& Weaver (1949) - mit zunehmender Abwasserbelastung herabgesetzt wird. Solche Reaktion ist aus anderen Untersuchungen über den Einfluß von Abwasser auf sublitorales, marines Benthos bekannt (Ludwig \& Storrs, 1970; Coastal Water Research Projekt, 1977). 
Die Fauna auf den Wattflächen verhält sich im Gegensatz zur Prielfauna indifferent, was sich besonders dadurch ausdrückt, daß sich in keinem Falle eine signifikante Korrelation nachweisen läßt und sogar negative Koeffizienten auftreten (Tab. 12). Der Abwassereinfluß führt innerhalb der Wattenbiozönosen also nicht zu Störungen der biotischen Mannigfaltigkeit, wenn man vom direkten Verschmutzungszentrum absieht, sondern die Diversität steigt sogar noch im Bereich mittlerer Verschmutzung an.

\section{Analyse der bestandsbildenden Arten und ibre Dominanz-Verbältnisse in den Bereichen unterschiedlicher Belastung}

Die (Individuen-)Dominanz gibt an, welchen numerischen Anteil eine Art - oder Gruppe - innerhalb einer Gesamtfauna an einer Station oder in einem Gebiet hat (Balogh, 1958). Es stellte sich die Frage, ob durch den Abwassereinfluß bestimmte Arten in den verschieden stark belasteten Zonen durch das Abwasser favorisiert, andere dagegen zurückgedrängt werden.

Am Beispiel des Einleitungsgebietes bei Kampen wurde eine Dominanzanalyse für die einzelnen Arten in den Zonen verschieden starker Verunreinigungen für beide Untersuchungsperioden (1970 und 1972/73) durchgeführt (Tab. 13 und 14). Die Rangfolge der Arten ergab sich aus dem Mittelwert der Individuenzahlen pro Quadratmeter im jeweiligen Transekt. Die zwei Wattentransekte (L- und M-Transekt) wurden zusammengefaßt und dem Abwasserpriel (K-Transekt) gegenübergestellt. Die Berechnungen der Dominanzen erfolgte unter Berücksichtigung aller Arten, die an wenigstens einer Station einen Anteil von $1 \%$ (Dominanz $=0,01)$ der hier lebenden Individuen erreichten, und an mindestens zwei Stationen der Priel- bzw. der beiden Wattentransekte vorkamen. Sie werden im folgenden bes $t$ a nd s bild end genannt; alle anderen Arten sind unter "Sonstige" zusammengefaßt.

Die Tabellen 13 und 14 erlauben den Vergleich der Beobachtungen aus zwei Untersuchungszeiträumen (1970 und 1972/73). Dieser zeigt die Reaktion des Benthos auf die verbesserte Abwasserklärung in der zweiten Untersuchungsperiode. Darauf wird noch besonders eingegangen.

Die Anzahl der bestandsbildenden Arten war für beide Untersuchungszeiträume im Abwasserpriel jeweils höher als auf den trockenfallenden Wattflächen, mit einem geringeren Unterschied im Untersuchungsjahr 1970 (Tab. 13 und 14). Die gleiche Beobachtung galt schon für die Gesamtartenzahlen: auch sie zeigten höhere Werte für den Priel (Tab. 10).

Aus der Rangfolge der Arten ergeben sich folgende hervorzuhebende Gemeinsamkeiten und Unterschiede für den Priel und die Wattflächen: (1) In beiden Untersuchungszeiträumen und beiden Teilbiotopen (Priel und Wattflächen) war Hydrobia ulvae die bei weitem individuenreichste Art und nahm somit von ihren Individuenzahlen her den ersten Rang ein. (2) Die Oligochaeten folgten auf dem zweiten Rang mit Ausnahme des Untersuchungsjahres 1970, in dem sie von Corophium volutator und Nereis diversicolor auf den Wattflächen an die vierte Stelle verdrängt wurden. (3) Die folgenden Ränge wurden in beiden Teilbiotopen von detritusfressenden Polychaeten und Macoma balthica eingenommen. (4) Macoma balthica spielte im Untersuchungsgebiet nicht die führende Rolle, 
die ihr sonst im Watt zukommt und nach der die Wattenbiozönose ihren Namen erhalten hat (Remane, 1940). (5) Capitella capitata, ein kosmopolitischer Abwasser-Indikator (Reish, 1955, 1959) trat gegenüber den typischen Wattenarten Scoloplos armiger, Eteone longa, Nereis diversicolor oder Arenicola marina zahlenmäßig zurück und erreichte lediglich $1970 \mathrm{im}$ Abwasserpriel nahe der TNW-Linie einen höheren Anteil, während dieser Polychaet auf den Wattflächen fast vernachlässigt werden konnte. (6) Die nicht bestandsbildenden Arten ("associated species"), in den Tabellen als "Sonstige" zusammengefaßt, erreichten im Priel zum Teil höhere Dominanz-Werte als auf den Wattflächen, auf denen sie ohne Bedeutung für Produktion und Biomasse waren.

Die Rangfolge der Arten, die keine prinzipiellen, sondern nur graduelle Unterschiede zwischen Abwasserpriel und trockenfallenden Wattflächen aufzeigt, läßt also keinen Einfluß des Abwassers auf die Zusammensetzung des Makrobenthos im Untersuchungsgebiet erkennen; die Fauna weist vielmehr - mit Ausnahme von Capitella capitata - eine für Schlickwatten typische Zusammensetzung auf.

Die Dominanz-Werte der bestandsbildenden Arten zeigen im Gegensatz zur Rangfolge (1) im Priel einen deutlichen Einfluß des Abwassers auf die prozentuale Zusammensetzung des Makrobenthos in den Zonen unterschiedlicher Abwasserbelastung und (2) die verschiedene Reaktion des Benthos in den beiden Teilbiotopen Abwasserpriel und Wattflächen.

In der starkbelasteten Zone des Prieles dominierten die individuenreichsten Arten des Transektes in beiden Untersuchungsperioden auch an den einzelnen Stationen (Tab. 13). Dabei beherrschten Hydrobia ulvae bzw. die Oligochaeten zum Teil mit über $90 \%$ (Dominanz-Wert $=0,9)$ der Individuen das Faunenbild. Erst mit abnehmender Verschmutzung erreichten die weniger individuenreichen Arten die höchsten DominanzWerte innerhalb der dortigen Benthos-Assoziationen (1970 ab Station K 10 und 1973 schon ab Station K 4), wobei einzelne Arten jedoch niemals über $50 \%$ der Individuen stellten.

Eine solche Sukzession der Individuen-Dominanz in Abhängigkeit von der Belastung war auf den Wattflächen (L- und M-Transekt) nicht festzustellen. Hier bestimmen jeweils zwei Arten die Makrofauna der gesamten Transekte: 1970 waren es hauptsächlich Hydrobia ulvae und Corophium volutator (in einigen Fällen auch $H$. ulvae und Nereis diversicolor bzw. Oligochaeten) und 1972 H. ulvae und Oligochaeten (in zwei Fällen auch Nereis diversicolor).

Wie für schlickartige Wattensedimente zu erwarten, setzten sich auf den Wattflächen also die Benthos-Gesellschaften aus wenigen detritusfressenden und sedimentabweidenden Arten zusammen; grundsätzliche Veränderungen durch das eingeleitete Abwasser waren nicht zu erkennen. Lediglich an den beiden an den Abwasserpriel grenzenden Stationen (L1: 1970/72; M1: nur. 1972) war das Makrobenthos auf nur eine Art (H. ulvae) beschränkt.

Insgesamt bestimmen also typische Wattarten die Benthos-Assoziationen im Einleitungsgebiet; selbst der Abwasserindikator Capitella capitata-keine typische Wattenform - kommt zwar in den abwasserbelasteten Zonen vor, kann sich aber nicht gegen die Wattarten durchsetzen und erreicht somit nur mittlere Dominanz-Werte. 


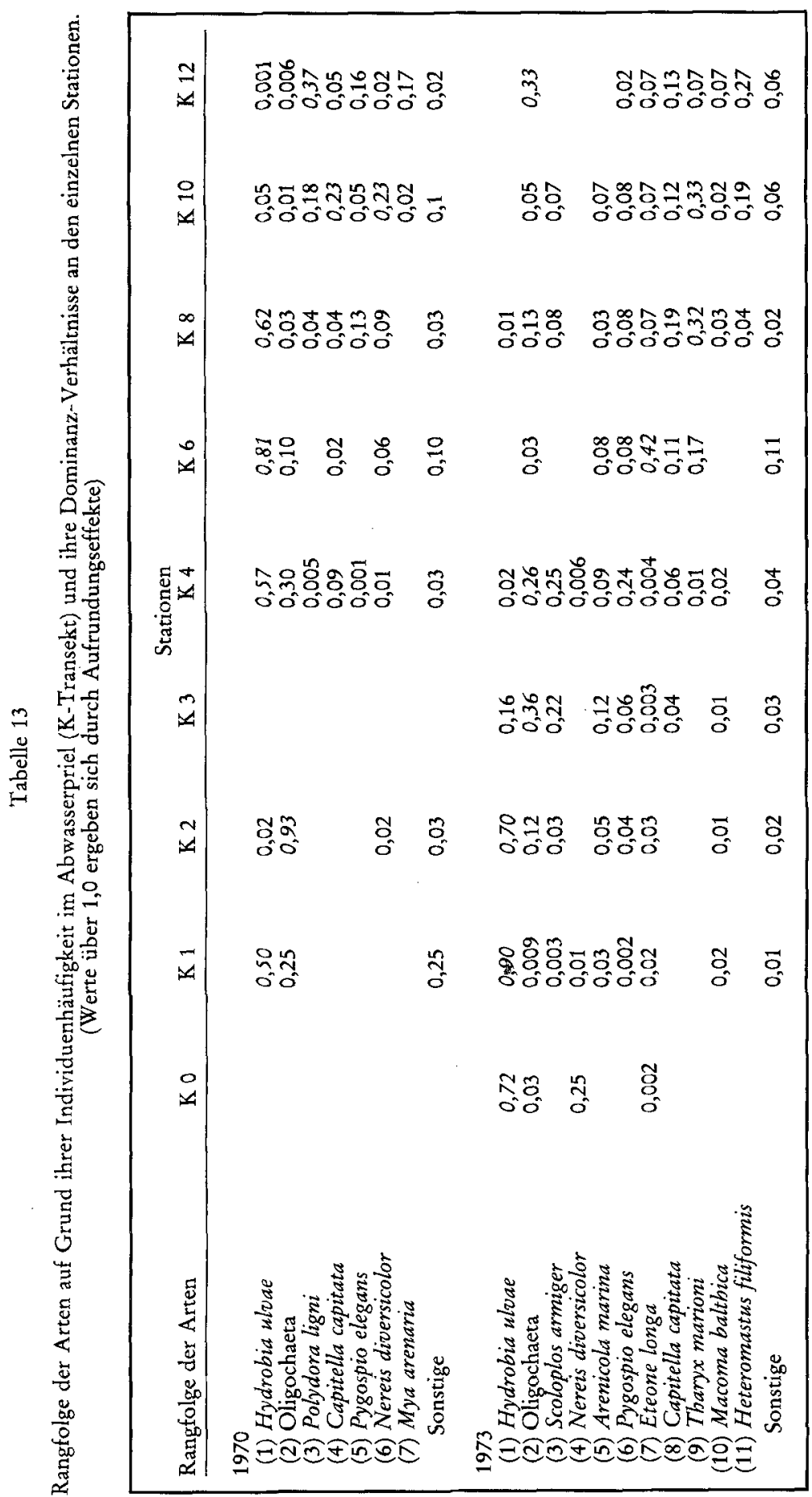


Auswirkungen kommunaler Abwässer auf marine Watten

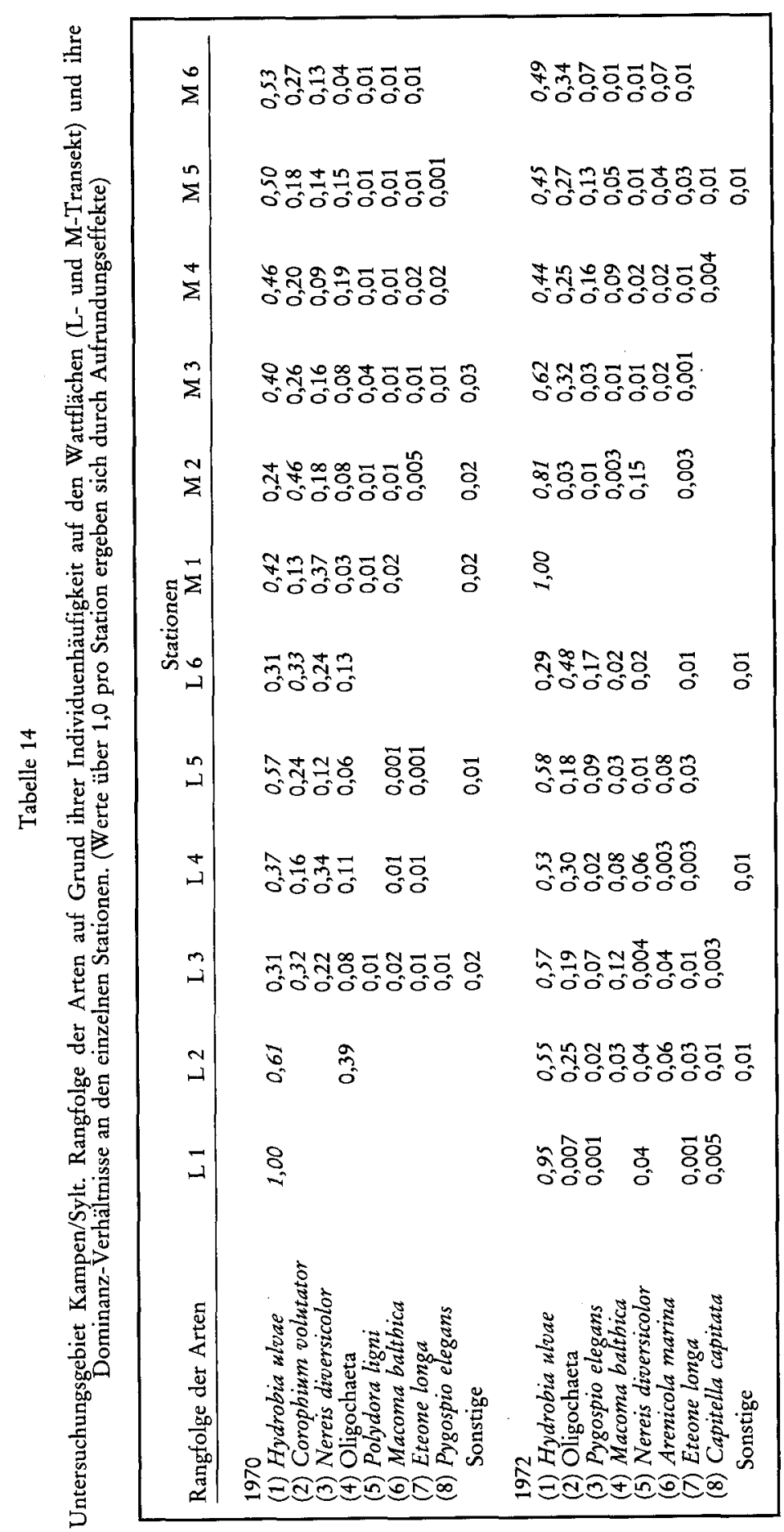


Einfluß einer verbesserten Abwasser-Vorreinigung auf das Benthos im "Vorfluter Watt"

Während der Untersuchungszeit im Wattengebiet von Kampen wurde das erweiterte und verbesserte Klärwerk in Betrieb genommen (s.o.). Damit war es möglich, die Auswirkungen einer verbesserten Abwasserklärung auf das Benthos zu erfassen.

Es sollte zunächst festgestellt werden, ob durch eine verbesserte Vorreinigung des Abwassers ein Faunenwechsel im Vorfluter-Watt eintrat. Dabei kam es nicht so sehr darauf an, nur die bestandsbildenden Arten innerhalb der Transekte vor und im Anschluß an die bessere Abwasserqualität $\mathrm{zu}$ vergleichen, sondern es sollten auch alle nur fleckenhaft vorkommenden, aber nicht extrem seltenen Arten mitberücksichtigt werden. Die Abbildung 20 enthält daher alle Arten, die im Durchschnitt pro Quadratmeter Sedimentoberflä-

\begin{tabular}{|c|c|c|c|}
\hline & $a$ & Abwasserpiel & \\
\hline 12606 & Hydrobia ulvae & - Hydrobia ulvae & 8983 \\
\hline 4481 & Otigochaeta & _ Oligochaeta & 1444 \\
\hline 2050 & Polydora ligni & * Scoloplos armiger & 772 \\
\hline 1600 & Capitelia capitata & Arenicola marina & 616 \\
\hline 1362 & Pygospio elegans & Pygospio elegans & 533 \\
\hline 1268 & Nereis diversicolo & Eteone longa & 361 \\
\hline 768 & Myo arenaria & Capitelia capitata & 261 \\
\hline 581 & Macoma balthica & Tharyx marioni & 255 \\
\hline 575 & Tharyx marioni & Mocoma balthica & 216 \\
\hline 56 & Arenicolc $\mathrm{m}$ & Nereis diversicolor & 72 \\
\hline
\end{tabular}

\begin{tabular}{|c|c|c|c|}
\hline & Wattflächen & $b$ & \\
\hline 11958 & Hycrobia uivce & Hydrobia uivae & 21595 \\
\hline 5775 & Corophium volutato & Oligochaeta & 6175 \\
\hline 4495 & Nereis diversicolo & Pygospio elegans & 1912 \\
\hline 3125 & Oligochaeta & Mocoma balthica & 1129 \\
\hline 254 & Polydora ligni & Nereis diversicolor & 827 \\
\hline 208 & Macoma balthica & Arenicola marina & 741 \\
\hline 200 & Eteone longa & Eteone longa & 333 \\
\hline 158 & Pygospio elegans & & \\
\hline \multirow[t]{2}{*}{37} & Capitella capitata & Capitella capitata & 96 \\
\hline & & Corophium valutator & 20 \\
\hline
\end{tabular}

Abb. 20: Untersuchungsgebiet Kampen/Sylt. Einfluß einer verbesserten Vorreinigung des Abwassers auf die Zusammensetzung der Makrofauna im Einleitungsgebiet. Neben den Arten die durchschnittlichen Individuenzahlen pro $\mathrm{m}^{2}$ Sedimentoberfläche. Die Pfeile deuten in Richtung der höheren Individuenzahl einer Art. Ohne Pfeil: bei der zweiten Untersuchung nicht mehr vorhandene oder neu hinzugekommene Arten. (a) Fauna bei der Einleitung von teilgeklärtem Abwasser, (b) Fauna bei der Einleitung von intensiv vorgereinigtem Abwasser

che mit mindestens 100 Individuen vorkamen. Die Rangfolge ergab sich entsprechend ihrer Häufigkeit. Wegen der unterschiedlichen Zusammensetzung der Fauna im Abwasserpriel und auf den Wattflächen sollen beide Teilbiotope getrennt betrachtet werden.

Es zeigte sich, daß im Priel auch bei besserer Abwasserqualität Hydrobia ulvae und die Oligochaeten am individuenreichsten blieben (Abb. 20), wenn auch ihre Abundanz abnahm. Sie sind bekannt für ihr Vorkommen in verunreinigtem Wasser (Blegvad, 1932; Fraser, 1932; Brinkhurst, 1966; Muus, 1967). Innerhalb der dann rangmäßig folgenden 
Arten traten mit der besseren Abwasserreinigung Änderungen ein: Polydora ligni, von Wass (1967) als Indikator für Verschmutzung angegeben, verschwand völlig und wurde durch Scoloplos armiger ersetzt, eine nach Anger (1975a) sogar teilweise Abwasserempfindlichkeit aufweisende Art. Capitella capitata, ein kosmopolitischer Indikator für organische Meeresverschmutzung (s. besonders Reish, 1955, 1957, 1959, 1960, 1970, 1972 u. a.), fiel vom vierten auf den siebten Rang, Hinweis auf eine verminderte Belastung. Dafür wurde Arenicola marina zahlreicher, eine nach Rosenberg (1971) gegen Verschmutzung empfindliche Art. Pygospio elegans, häufig auch als Indikator für Verschmuzung aufgeführt (Clay, 1967a; Anger, 1975a, 1977), behielt den gleichen Rang, wenn auch mit reduzierter Individuenzahl. Nereis diversicolor und Mya arenaria nahmen sehr stark ab, während Macoma balthica und Tharyx marioni etwa ihre Stellung innerhalb der Rangfolge behaupteten, allerdings mit deutlich verminderten Individuendichten. Als neue Art hinzu kam nur Eteone longa. Nach Untersuchungen von Anger (1975a) ist diese Art nicht als Indikator für eine bestimmte Wasserqualität geeignet, da sie zu unregelmäßig vorkommt. Im Untersuchungsgebiet Kampen mied sie allerdings den unmittelbaren Einleitungsbereich. Tulkki (1968) bezeichnet Eteone longa sogar als regressive Art in bezug auf Verschmutzung.

Wie die Analysen zeigten, hatte im Abwasserpriel infolge der verbesserten Vorreinigung des Abwassers ein partieller Wechsel zugunsten weniger resistenter Arten stattgefunden; Verschmutzungsindikatoren, wie Capitella capitata, Polydora ligni, und Macoma balthica (Stephen, 1929; Fraser, 1932; Tulkki, 1969), wurden seltener oder verschwanden ganz aus den Benthos-Assoziationen. Alle Arten, auch wenn sie noch den gleichen Rang einnahmen, wiesen außerdem reduzierte Individuenzahlen auf. Diese Veränderungen sind Anzeichen dafür, daß die Beeinflussung der Umweltfaktoren im Priel abgenommen hatte und insbesondere das Nahrungsangebot zurückgegangen war.

Auf den trockenfallenden Wattflächen war 1973 ebenfalls Hydrobia ulvae weiterhin am individuenreichsten vertreten, sogar mit wesentlich höheren Siedlungsdichten als noch 1970 vor dem Ausbau der Kläranlage (Abb. 20). Im Gegensatz zum Abwasserpriel charakterisierten dann aber die Oligochaeten, Pygospio elegans und Macoma balthica wegen der Zunahme ihrer Individuenzahlen das Benthos. Auch Capitella capitata wurdewenn auch zu einem geringeren Grad - häufiger. Alle diese Faunenelemente sind bekannt für ihre Abwasserverträglichkeit. Dagegen nahm Nereis diversicolor in seinem Vorkommen sehr stark ab. Coropbium volutator, 1970 noch mit großen Individuendichten hinter Hydrobia ulvae die zweithäufigste Art auf den Wattflächen, verschwand erstaunlicherweise fast völlig, siedelte 1975 allerdings wieder in großen Mengen in diesem Gebiet. Den Grund für diese Fluktuationen in der Änderung der Abwasserqualität zu suchen, scheint fraglich, da Corophium volutator an Fäulnisprozesse in Sedimenten der Schlickwatten adaptiert ist und außerdem häufig an Einleitungsstellen für Abwasser vorkommt (s. Funde bei Kampen, Braderup sowie Fraser, 1932; Clay, 1967b). Ebenso wie im Priel verschwand Polydora ligni von den Wattflächen und Arenicola marina wurde häufiger. Neben den Hydrobien blieb nur Eteone longa auf dem gleichen Rang wie 1970, aber auch mit höheren Individuenzahlen.

Auf dem Watt war also durch die verbesserte Abwasserklärung bei den meisten Arten eine Zunahme der durchschnittlichen Individuendichte zu verzeichnen; an Verschmutzung adaptierte Formen traten in den Vordergrund. Bei dieser zum Abwasserpriel konträren 
Reaktion des Benthos mag eine Rolle gespielt haben, daß 1972 eine hohe Besiedlung des Sedimentes wegen des geringeren Abwassereinflusses bis an das Verschmutzungszentrum möglich war. Dadurch konnte flächenmäßig das durch sedimentierte Abwasserpartikel erhöhte Nahrungsangebot ausgenutzt werden. 1970 dagegen war hier ein größerer Bereich unbesiedelt geblieben.

Die bessere Vorreinigung des eingeleiteten Abwassers verursachte nicht nur einen partiellen Faunenwechsel. Ebenso war ein Einfluß auf die Verteilung der Arten- und Individuenzahlen des Makrobenthos in Relation zur abnehmenden Belastung innerhalb der Transekte nachzuweisen. Es ergaben sich folgende Veränderungen: (1) Die Degradationszone im Verschmutzungszentrum wurde so stark reduziert, daß sie nur noch durch eine geringfügige Abnahme der Arten- und Individuenzahlen zu erkennen war (Abb. 14 und 16). (2) Das Individuenmaximum war im Priel zur Einleitungsstelle hin verschoben; die Artenvielfalt stieg schneller an. (3) Auf den Wattflächen waren Arten- und Individuenmaxima gemeinsam zum Verschmutzungszentrum hin verlagert. (4) Die Siedlungsdichte des Makrobenthos war 1972/73 generell niedriger als 1970, wobei auf den Wattflächen zusätzlich das Artenspektrum drastisch reduziert war.

Das intensiver vorgereinigte Abwasser beeinflußte durch die Veränderungen in der Verteilung der Benthos-Assoziationen indirekt auch die biotische Mannigfaltigkeit der Makrofauna. So nahmen 1972/73 die Diversitätsindizes im Abwasserpriel (K-Transekt) und im L-Transekt auf den Wattflächen zunächst mit wachsender Entfernung vom Verschmutzungszentrum schneller zu als 1970. Sie pendelten sich dann aber schon in geringerer Entfernung von der Einleitungsstelle auf annähernd gleich hohe Werte ein, erreichten allerdings nicht ganz die Maximalwerte von 1970 (Abb. 18 und 19). Nur im MTransekt auf den Wattflächen stieg die biotische Mannigfaltigkeit trotz des besser vorgeklärten Abwassers langsamer an als vorher. Die Korrelation zwischen Diversitätsindizes und Abwasserbelastung im Priel schwächte sich gegenüber 1970 durch die geringere Verschmutzung leicht $a b$, blieb aber signifikant. Für die Wattflächen war keine eindeutige Beziehung zwischen beiden Komponenten festzustellen (Tab. 12); allerdings erhöhte sich der Koeffizient für den M-Transekt.

\section{Adaptationsfäbigkeit des Makrobenthos für Veränderungen des Sedimentes bei Abwassereinleitungen}

Fauna-Sediment-Beziehungen sind schon häufig untersucht worden (z. B. von Sanders, 1958; McNulty et al., 1962; Rhoads \& Young, 1970; Johnson, 1971; Bloom et al., 1972; Gray, 1974). Alle Autoren stellten heraus, daß viele Benthos-Arten nur in definierten Sedimenttypen vorkommen und dort typische Faunen-Assoziationen bilden.

In Verbindung mit Abwassereinleitungen in Wattengebiete kommt es häufig zu direkten und indirekten Sedimentveränderungen. Als mögliche Ursachen seien genannt: Abgrenzungen des Vorfluterbereiches mit Molen, Ausbaggern oder Ausspülen eines Abwasserkanals und verstärkte Aufschlickungsraten durch das Sedimentieren von Abwasserpartikeln.

Es stellte sich die Frage, ob die in Einleitungsgebieten anzutreffenden MakrobenthosArten eine erhöhte Toleranz gegenüber variierenden Sedimentfaktoren aufweisen oder sich 
in den Sedimentations-Zonen eine gegenüber Schlick und Detritus stenöke Fauna ansiedelt. Daher sollte untersucht werden, welche Sedimente im Einleitungsgebiet von den meisten Arten bevorzugt werden, ob und wie viele von den Arten mit Präferenzen für definierte Sedimenttypen auch in anderen Sedimenten vorkommen und wie groß die Toleranzbreite der einzelnen Arten und Artengruppen gegenüber der Korngrößenzusammensetzung und der sedimentären Detritus-Anteile ist.

Als Modell für abwasserbelastete Watten bot sich das Untersuchungsgebiet Vancouver an, da hier alle Sedimentformen vom Schlick bis zum Grobsand vorkamen.

Das Sediment wurde als Schlick bezeichnet, wenn die Silt-Ton-Fraktion größer als $50 \%$ war, als Schlicksand, wenn ihr Anteil zwischen 3 und $50 \%$ betrug und als Sand bei einem Silt-Ton-Wert von weniger als $3 \%$. Entsprechend dieser Einteilung wiesen 12 Stationen Schlick, 23 Stationen Schlicksand und 7 Stationen Sand auf.

Alle Makrobenthos-Arten, die an mindestens drei Stationen vorkamen, ließen sich entsprechend ihrer Individuenabundanzen drei Arten- und Faunengruppen zuordnen: den Schlick-Arten, den Schlicksand-Arten und den Sand-Arten.

Um zu ermitteln, wie groß die Toleranz gegenüber Sedimentveränderungen ist, wurde die Variabilität der Korngrößenverteilung und der sedimentären Detritusanteile für die einzelnen Arten und die Artengruppen berechnet. Dabei wurden die Sedimente von allen Stationen, an denen die betreffende Art oder Gruppe vorkam, berücksichtigt, unabhängig

Tabelle 15

Variabilitätskoeffizient der mittleren Korngrößenverteilung und des Detritusanteils der Sedimente für die drei Artengruppen. $V_{1}=$ Koeffizient der Korngrößenverteilungen, $V_{2}=$ Koeffizient der Detritusanteile

\begin{tabular}{|lcc|}
\hline Artengruppen & $\mathrm{V}_{1}$ & $\mathrm{~V}_{2}$ \\
\hline Schlick-Arten & 54,6 & 15,5 \\
Schlicksand-Arten & 50,7 & 45,7 \\
Sand-Arten & 39,3 & 20,6 \\
\hline
\end{tabular}

von ihren Abundanzen. Die Standardabweichung (s) für den Mittelwert der Einzeldaten ist normalerweise ein geeignetes Maß, um die Variabilität eines Merkmals anzugeben. $D a$ es jedoch vom absoluten Betrag des Mittelwertes abhängt, erlaubt die Standardabweichung keinen Vergleich unterschiedlicher Merkmale. Es wurde daher der Variabilitätskoeffizient (V), der s in Prozent vom Mittelwert angibt, zur Charakterisierung der Arten-Toleranz benutzt (Steel \& Torrie, 1960; Weber, 1967; Cavalli-Sforza, 1968; Sachs, 1971).

Für die Variabilitätsberechnungen wurden insgesamt 20 Arten berücksichtigt. Nur 3 Arten hatten ihr Hauptvorkommen im Schlick, von denen 2 auch im Schlicksand und 2 im Sand zu finden waren. Ihr Optimum im Schlicksand hatten 13 Arten. Alle kamen auch im Schlick vor, aber nur 8 im Sand; von 4 Sand-Arten erschienen jeweils zwei auch im Schlick und Schlicksand.

Die Schlicksand-Fauna zeigt also durch ihr geschlossenes Vorkommen im Schlick die größte Toleranz gegenüber einem anderen Sedimenttyp. Für sie stellt eine begrenzte Aufschlickung folglich keine existenzbedrohende Gefährdung dar, während die SandArten schlickige Sedimente völlig meiden. 
Tabelle 16

Mittlere Sedimentmediane $(\mu \mathrm{m})$ und Mittelwerte der partikulären organischen Substanz $(\%)$ sowie deren Variabilitätskoeffizienten $(\mathrm{V}, \%)$ für die Arten der drei Faunengruppen. $\mathrm{V}_{1}=$ Koeffizienten der Korngrößenverteilungen, $V_{2}=$ Koeffizienten der Detritusanteile

\begin{tabular}{|c|c|c|c|c|}
\hline Species & Medianwert & $\mathrm{V}_{1}$ & org. Substanz & $\mathrm{V}_{2}$ \\
\hline \multicolumn{5}{|l|}{ Schlick-Arten: } \\
\hline $\begin{array}{l}\text { Glycinde picta } \\
\text { Paraonis platybranchia } \\
\text { Anisogammarus confervicolus }\end{array}$ & $\begin{array}{c}179 \\
146 \\
47,6\end{array}$ & $\begin{array}{l}72,7 \\
57,2 \\
34,0\end{array}$ & $\begin{array}{l}3,08 \\
1,79 \\
2,72\end{array}$ & $\begin{array}{r}12,7 \\
7,3 \\
26,5\end{array}$ \\
\hline \multicolumn{5}{|l|}{ Schlicksand-Arten: } \\
\hline $\begin{array}{l}\text { Eteone longa } \\
\text { Nereis limnicola } \\
\text { Barantolla americana } \\
\text { Polydora kempi japonica } \\
\text { Pygospio elegans } \\
\text { Manayunkia aestuarina } \\
\text { Amphicteis spec. I } \\
\text { Oligochaeta } \\
\text { Nemertini } \\
\text { Corophium salmonis } \\
\text { Tanais spec. } \\
\text { Macoma inconspicua } \\
\text { Mya arenaria }\end{array}$ & $\begin{array}{c}109 \\
73,5 \\
126,6 \\
104,3 \\
94 \\
60,3 \\
91,5 \\
108,8 \\
107,3 \\
78,7 \\
48,3 \\
103,6 \\
95,2\end{array}$ & $\begin{array}{l}45,9 \\
34,8 \\
35,4 \\
60,6 \\
46,4 \\
45,8 \\
59,2 \\
66,4 \\
62,5 \\
48,5 \\
42,0 \\
48,2 \\
52,9\end{array}$ & $\begin{array}{l}2,74 \\
1,91 \\
1,98 \\
2,37 \\
2,28 \\
2,89 \\
2,2 \\
2,46 \\
2,04 \\
2,14 \\
2,2 \\
2,49 \\
2,07\end{array}$ & $\begin{array}{l}78,1 \\
23,6 \\
33,3 \\
51,1 \\
68,9 \\
58,8 \\
36,4 \\
54,9 \\
29,4 \\
37,9 \\
20,0 \\
68,7 \\
32,4\end{array}$ \\
\hline \multicolumn{5}{|l|}{ Sand-Arten: } \\
\hline $\begin{array}{l}\text { Clinocardium nuttalli } \\
\text { Eobaustorius washingtonianus } \\
\text { Paraphoxus spec. } \\
\text { Lamprops spec. }\end{array}$ & $\begin{array}{l}228 \\
128,2 \\
139 \\
118\end{array}$ & $\begin{array}{l}23,7 \\
22,1 \\
47,6 \\
63,8\end{array}$ & $\begin{array}{l}2,09 \\
1,73 \\
2,07 \\
1,74\end{array}$ & $\begin{array}{r}24,9 \\
19,7 \\
33,3 \\
4,6\end{array}$ \\
\hline Capitella capitata & 108 & 91,4 & 3,79 & 83,9 \\
\hline
\end{tabular}

Eine unterschiedliche ökologische Potenz der drei Artengruppen zeigen auch die Variabilitätskoeffizienten für Korngrößenzusammensetzung und Detritusgehalt der von ihnen besiedelten Sedimente (Tab. 15). Ein Einfluß der Korngröße ist nur bei der Sandfauna einschränkend wirksam, während die beiden anderen Gruppen für diesen Faktor eine Variabilität von über $50 \%$ zeigen, also keine hohe Affinität zu einer bestimmten Korngrößenverteilung haben.

Größere Unterschiede sind in der Reaktion der Artengruppen gegenüber dem Gehalt an organischem Restmaterial zu konstatieren. Während die Sand-Arten und noch mehr die Schlick-Arten in ihrer Variabilität gegenüber Detritus mit einem Koeffizient von 20,6\% bzw. 15,5\% als stenök zu bezeichnen sind, zeigt der Wert für die Schlicksand-Fauna von 45,7\% die hohe Adaptationsfähigkeit dieser Artengruppe.

$\mathrm{Daß}$ Arten, die gegenüber verschiedenen Korngrößen der Sedimente tolerant sind, nicht notwendigerweise zugleich auch unterschiedlich hohe Detritus-Anteile tolerieren, zeigt Tabelle 16: Für das Vorkommen von Schlick-Arten sind die Korngrößen bedeutungslos, wie aus den sehr hohen Variabilitäts-Koeffizienten hervorgeht; organischem Material 
gegenüber verhalten sich die Elemente dieser Faunengruppe jedoch stenök. Nur Anisogammarus confervicolus ist Detritus gegenüber variabler, benötigt dafür aber enger definierte Korngrößen. Dagegen weisen viele Schlicksand-Arten gegenüber den beiden Sedimentfaktoren, Korngrößenzusammensetzung und Detritusgehalt, eine hohe Variabilität auf und sind damit für Sedimentveränderungen praedisponiert. $\mathrm{Zu}$ ihnen gehören Arten, die im Untersuchungsgebiet am weitesten verbreitet waren und gleichzeitig hohe Abundanzen erreichten: Eteone longa, Polydora ligni, Macoma inconspicua, Mya arenaria, Nemertini und Oligochaeta, Capitella capitata, Pygospio elegans, Manayunkia aestuarina und Amphicteis spec. I (mit Ausnahme von Corophium salmonis). Eine leichte Präferenz für ein bestimmtes Sediment mit einem definierten Anteil an organischen Partikeln zeigen lediglich Nereis limnicola und Barantolla americana. Abweichungen von etwa $30 \%$ werden dabei noch toleriert.

Die am wenigsten flexiblen Arten werden durch Elemente der Sand-Fauna repräsentiert; für beide Sedimentfaktoren wurde eine geringe Variabilität festgestellt. Nur Lamprops spec. zeigte ein anderes Verhalten: Während diese Cumacee Sedimente sehr unterschiedlicher Korngrößenverteilungen tolerierte, akzeptierte sie dagegen Änderungen im Detritusgehalt nur von $4,6 \%$.

Capitella capitata wäre auf Grund von zwei Massenvorkommen im groben Sediment als Sand-Art einzustufen, war aber in etwa gleicher Abundanz an Stationen mit Schlick und Schlicksand zu finden. Die Art ist deshalb als Ubiquist anzusehen. Sie erreichte die bei weitem höchsten Variabilitäts-Koeffizienten für Korngrößen und organische Substanz. Ihr Vorkommen hängt also nicht von Sedimentfaktoren ab.

\section{DISKUSSION}

Die vorliegenden Untersuchungen zeigen, daß eine Einleitung kommunaler Abwässer zu deutlichen Effekten in Wattengebieten führt. Nachweisen ließen sich Auswirkungen auf (1) die abiotischen Umweltfaktoren, (2) die Primärproduzenten und die Destruenten im Sediment und (3) das Makrobenthos. Es erscheint daher sinnvoll, die Ergebnisse für diese drei untersuchten Teilaspekte des Okosystems Wattenmeer nacheinander zu diskutieren.

\section{Uber die natürliche Schwankungsbreite hinausgehende Veränderungen der abiotischen Umweltfaktoren}

Bei Niedrigwasser beschränkt sich der direkte Abwassereinfluß auf den Kanal oder Priel, in dem das Abwasser während dieser Zeit abfließt; von den Wattflächen kommt nur ein schmaler Streifen kurzzeitig beim Auf- und Ablaufen des Meerwassers in direkten Kontakt mit dem Abwasser. Durch ein vorübergehendes Aufstauen im Priel wird es über die benachbarten Sedimente verteilt. Bei Hochwasser schichtet es sich an der Oberfläche ein. Den Benthos-Organismen steht daher in der Regel bei Uberflutung Seewasser mit normalem pH-Wert sowie Salz- und Sauerstoffgehalt zur Verfügung. Dadurch erklärt sich zum Teil die geringe Ausdehnung der Degradationszone, die besonders auf den Wattflächen in Relation zur eingeleiteten Abwassermenge stark reduziert ist. Ein Abwassereinfluß 
bleibt außerhalb des Priels und seiner Randstreifen auf die Sedimentation des eingeschwemmten Sestons in ufernahen Bereichen beschränkt. Da in Wattengebieten ein landwärtsgerichteter Transport von Trübstoffen stattfindet (Postma, 1960; DeJonge \& Postma, 1974), wird das Seston in der Regel mit dem vom Meer herangeführten Detritus nicht ins offene Meer abtransportiert.

Durch die Dekomposition des sedimentierten organischen Sestonanteiles kann es an der Sedimentoberfläche zu kritischen Sauerstoffverhältnissen kommen (O'Connor, 1972). Im Untersuchungsgebiet bei Vancouver wurde eine maximale Sauerstoffaufnahme des Sedimentes von $371 \mathrm{mg} \mathrm{O} \mathrm{O}_{2} / \mathrm{m}^{2} / \mathrm{h}$ gemessen (B. C. Research, 1975). Dieser Wert ist gegenüber unbelasteten Watten um den Faktor 2 bis 3 erhöht (Duff \& Teal, 1965; Pamatmat, 1968). Die 48stündigen $\mathrm{O}_{2}$-Dauermessungen in der Sediment-Wasser-Kontaktzone zeigen, daß für so hohe Zehrungsraten der "Nachschub" an Sauerstoff aus dem darüberfließenden Wasser nicht mehr ausreicht und anaerobe Verhältnisse resultieren. Eine höhere Strömungsgeschwindigkeit des Wassers, die ein verstärktes $\mathrm{O}_{2}$-Angebot bedeuten würde, führt jedoch in Bereichen, wo feinste Abwasserpartikel sedimentieren, zum Aufwirbeln von Detritus. Dadurch steigt die Zehrungsrate in der Kontaktzone noch an. Die langen Zeiten ohne Sauerstoff über dem Sediment waren (besonders bei Station 3: Tab. 3; Abb. 7) darauf zurückzuführen. Diese Beobachtungen stimmen gut mit den Untersuchungen von Knowles et al. (1962), Martin \& Bella (1971) und Pamatmat (1971) überein; sie fanden, daß die Sauerstoffaufnahme von Sedimenten stark ansteigt, wenn das darüberstehende Wasser bewegt wird. Edwards \& Rolley (1965) und Frankenberg \& Westerfield (1968) ermittelten, daß ein Aufwirbeln von Sediment hohe $\mathrm{O}_{2}$-Defizite im Wasser über dem Sediment verursachen kann. Pamatmat konnte außerdem zeigen, daß die $\mathrm{O}_{2}$-Aufnahme des Sedimentes sehr stark ansteigt, wenn die obere oxydierte Schicht weggespült und dadurch die Reduktionsschicht exponiert wird.

Die zeitweilig völlige Abwesenheit von Sauerstoff in der Sediment-Wasser-Kontaktzone im Bereich der Kläranlageneinleitung muß folglich auf eine additive Wirkung dreier Faktoren zurückgeführt werden: (1) eine höhere Sauerstoffaufnahmerate der Sedimente durch die Wasserbewegung, (2) das Aufwirbeln von feinen Partikeln und (3) die dadurch bedingte Exposition von reduzierten Sedimenten.

Wieviel von der gesamten Sauerstoff-Zehrung dabei auf die chemische Oxydation und wieviel auf die Respiration entfällt, konnte bei den in situ-Versuchen nicht ermittelt werden. Pamatmat (1968) wies nach, daß die chemische Oxydation einen hohen Anteil an der Gesamtzehrung des Sedimentes hat: bei Luft-Exposition der Wattflächen wurden zwischen $30 \%$ und $51 \%$ des von ungestörten Sedimentoberflächen aufgenommenen Sauerstoffs für chemische Reaktionen verbraucht.

Ein Einfluß auf die Sedimentstruktur im Einleitungsgebiet ist nur durch Veränderungen der Strömungsverhältnisse möglich. In Zonen, die einer natürlichen Aufschlickung unterliegen, tragen die partikulären Abwasserinhaltsstoffe zu einer höheren Sedimentationsrate bei. So wird z. B. im Untersuchungsgebiet List/Sylt das Abwasser in eine Bucht eingeleitet, die von einer hakenförmigen Sanddüne begrenzt ist. Das ehemalige Grobsandwatt unterliegt durch die Verminderung der Tidenströmungen einer Verschlickung, die vom Abwasser lediglich verstärkt wird. Dagegen sind die hohen Detritusanteile bei Vancouver durch den Bau der Nordmole bedingt; sie änderte die Strömungsverhältnisse so stark, daß aus einem Sandwatt, das nördlich der Mole noch vorhanden ist, ein Schlick- und 
Schlicksandwatt wurde. Die natürliche Verschlickung wird allerdings auch hier durch die Abwassereinleitung beschleunigt. Dies zeigt sich an dem teilweise extrem hohen Gehalt organischer Substanz im Sediment (bis zu 10,1\%).

Detritusanteile von über $5 \%$ sind in unverschmutzten Watten selten (Anderson, 1972; Levings \& Coustalin, 1975). Sogar in Klärschlamm-Verklappungsgebieten erreicht das organische Material selten so hohe Anteile. Shelton (1971) ermittelte Maxima von $5,7 \%$ bzw. $3 \%$ aus dem Themse-Aestuar, obwohl er seine Proben bei $600^{\circ} \mathrm{C}$ veraschte und dadurch vermutlich zu hohe Werte erhielt. Gross (1972) fand durchschnittliche Werte von $6 \%$ mir Maxima von 13,8\% in der Bucht von New York und meint, daß Werte von über $5 \%$ eindeutig auf den Einfluß von Klärschlamm zurückzuführen sind. Watling et al. (1974) analysierte maximal 3,6\% organisches Material im Klärschlamm-Verklappungsgebiet der Delaware Bay, USA, und Anderson (1972) maximal 3,7\% (geglüht bei $500^{\circ} \mathrm{C}$ für 2 Std.) in den Wattengebieten in Morecambe Bay, U. K., in der Nähe einer Abwassereinleitungsstelle. Nur in abwasserbelasteten Buchten, die zudem einen schlechten Wasseraustausch aufweisen, werden leicht $10 \%$ und mehr organisches Material im Sediment erreicht (Nichols, 1964; O'Connor, 1972).

Unter der Schlickschicht fand sich sowohl bei List/Sylt als auch bei Vancouver häufig Grobsand, der auf das ehemalige Sandwatt hinweist. Dadurch ergaben die Sedimentanalysen trotz hoher Detritusanteile teilweise auch hohe Medianwerte für die Korngrößenverteilung. In der Regel ist in Sedimenten aber der Anteil des organischen Materials mit dem Anteil der feineren Fraktionen positiv korreliert (Gadow, 1970), weil beide Faktoren gleichermaßen von der Strömungsgeschwindigkeit des Wassers abhängig sind. Ein Vergleich der vorliegenden Sedimentanalysen mit denen anderer Autoren für unbelastete Wattengebiete ist schwierig, da selten angegeben wird, bis zu welcher Tiefe und ob von ungestörten oder durchmischten Sedimenten die Proben für die Analysen entnommen wurden. Dies ist aber gerade bei schlecht sortierten Böden, wie sie z, B. in den Untersuchungsgebieten vorherrschen, zu Vergleichszwecken unbedingt notwendig.

Reaktion der Primärproduzenten und der Destruenten im Sediment auf künstliche Trophie- und Saprobitätserhöhungen

Da der Wasserkörper im Watt kontinuierlich ausgetauscht wird, läßt sich ein Eutrophierungseffekt des Abwassers nur für das Sediment im Vorfluter nachweisen: Die Biomasse sowohl der Cyanophyceen als auch der Diatomeen (als Chlorophyll-Menge im Oberflächensediment) wird in Abhängigkeit vom Verschmutzungsgradienten erhöht. Vergleiche der vorliegenden Analysenergebnisse mit den Untersuchungen anderer Autoren aus unbelasteten Biotopen ist nur bedingt möglich, da (1) identische Wattensedimente bisher nur wenig auf Chlorophyll und seine Abbauprodukte analysiert wurden (z. B. Leach, 1970), (2) die Art des Sedimentes nicht angegeben wird, (3) die Pigmentmenge auf das Trockengewicht des Sedimentes, statt auf die für in situ-Verhältnisse relevantere Volumen- oder Flächeneinheit bezogen (Cadée \& Hegeman, 1974) oder (4) nur die Menge an Gesamtpigmenten angegeben werden (Tietjen, 1968).

Die bei Sylt und Vancouver gefundenen absoluten Chlorophyll-Mengen liegen im allgemeinen in der Größenordnung der von Pamatmat (1968) in Watten gefundenen Werte, 
mit Ausnahme der Maximalwerte im unmittelbaren Einleitungsbereich. Diese von den Ergebnissen Pamatmats abweichenden hohen Werte müssen auf den Einfluß der Abwässer zurückgeführt werden. Auch das Vorkommen von Cyanophyceen in den Gebieten bei Sylt und Vancouver zeigt den Abwassereinfluß. Schon König (1968) stellte fest, daß Blaualgen durch Abwassereinleitungen gefördert werden.

Tietjen (1968) und Patterson \& Parson (1963) fanden in Sedimentproben flacher Küstengewässer generell höhere Phaeophytin a- als Chlorophyll a-Mengen. Daß auf den Wattsedimenten im Untersuchungsgebiet bei Kampen und Vancouver, mit Ausnahme einer schmalen Zone, signifikant höhere Chlorophyll a-Werte gemessen wurden, dürfte auf den Düngungseffekt des Abwassers und die optimalen Biotopverhältnisse zurückzuführen sein: hohes Nährstoffangebot aus dem Abwasser bei guter Belüftung und - besonders bei Niedrigwasser - guten photischen Verhältnissen. Der starke Lichteinfluß kann von den benthischen Algen voll ausgenutzt werden, da sie im Gegensatz zu planktischen Formen resistent gegen hohe Lichtintensitäten sind. Daher kommt es auch während der Expositionszeit nicht zu einer Photoinhibition der Algen (McMillan \& Verduin, 1953; Odum, 1957; Burkholder et al., 1965; Pamatmat, 1968; Gargas, 1971).

Die Bereiche, in denen eine Biostimulation der Algen festzustellen ist, liegen eng neben den Zonen, in denen sie durch das Abwasser zeitweise vernichtet werden. Diese Schädigungszonen sind nachzuweisen durch beträchtlich erhöhte Phaeophytin a-Werte. Tietjen (1968), der mit ästuarinen Sedimenten arbeitete, meint zwar, daß hohe Phaeophytin-Werte Areale anzeigen, an denen die Deponie von allochthonem suspendierten Material und das Zurückhalten von autochthonen Substanzen favorisiert werden; dies trifft jedoch für die Untersuchungsgebiete Kampen und Vancouver nicht zu. Wahrscheinlicher ist, daß es an den Orten mit höherem Phaeophytin-Gehalt durch wechselnden Abwassereinfluß häufig zum Absterben der Algen und zur raschen Rekolonisierung kommt, wodurch sich mit der Zeit die Abbauprodukte dort ansammeln. In der Regel fallen diese Zonen mit dem Wechsel von Cyanophyceen- zu Diatomeen-Gesellschaften zusammen; für beide Gruppen ist hier also die Verbreitungsgrenze. Zur Stützung dieser Hypothese sind aber noch weitere Untersuchungen nötig.

Mehrere Autoren haben aktives Chlorophyll a - also lebende Mikroalgen - unterhalb der euphotischen Zone festgestellt (Moul \& Mason, 1957; Grontved, 1960, 1962; Hopkins, 1963; Taylor \& Gebelein, 1966; Pamatmat, 1968; Leach, 1970; Fenchel \& Staarup, 1971), die in Sedimenten 2 bis $5 \mathrm{~mm}$ beträgt (Taylor \& Gebelein, 1966). Cadée \& Hegeman (1974) fanden in holländischen Watten bis in eine Tiefe von $10 \mathrm{~cm}$ gut nachweisbare Mengen von Chlorophyll a. Wenn die Algen von unterhalb der euphotischen Zone ans Licht gebracht wurden, zeigten sie nach Grøntved (1962) und Pamatmat (1968) sofort Photosynthese; sie bilden also ein Reservoir für die Sedimentoberfläche, falls hier die Algen vernichtet oder weggespült werden. Dadurch stabilisieren sie den Biotop. Als Auslösefaktoren für Vertikalwanderungen nimmt Hopkins (1963) Umweltveränderungen an. Es lag daher nahe, die Tiefenverteilung der Algen als Indikator für die Belastung von Wattsedimenten heranzuziehen. Der Vergleich der Vertikalverteilung des Chlorophyll a im Untersuchungsgebiet Kampen mit den Ergebnissen von Cadée \& Hegeman (1974) zeigt aber, daß in unbelasteten Watten ebenfalls die bei weitem höchsten Werte an der Oberfläche zu finden sind und die Pigmentmenge bis auf $5 \mathrm{~cm}$ Tiefe abfällt. Die vorliegenden Ergebnisse lassen also keinen Unterschied erkennen, durch den ein Abwassereinfluß nachzuweisen sein würde. 
Die benthischen Mikroalgen reagieren außer durch die Biomassenerhöhung auch durch intensivere Produktionsraten positiv auf das Abwasser. Im Vergleich zu Produktionsmessungen von Grøntved $(1960,1962)$ in dänischen Flachwassergebieten und von Leach (1970) in schottischen Wattengebieten (beide Autoren geben ebenfalls Produktionsraten pro $\mathrm{m}^{2}$ Sedimentoberfläche und Stunde an, benutzten aber unterschiedliche Methoden) liegen die Raten der vorliegenden Untersuchungen zumindest im Sommerhalbjahr im Durchschnitt über denen beider Autoren. Lediglich Grøntved $(1960,1962)$ konnte an einigen abwasserbeeinflußten Probenstellen Produktionswerte messen, die noch höher als die bei Kampen gefundenen Maximalwerte liegen (über $300 \mathrm{mg} \mathrm{C} / \mathrm{m}^{2} / \mathrm{h}$ ).

Da die benthischen Mikroalgen nach der Plankton-Methode sowie unter optimalen Wasser- und Lichtbedingungen und jeweils zur Zeit des höchsten Sonnènstandes inkubiert wurden, ist eine Übertragung der gemessenen Produktionswerte auf in situ-Verhältnisse nicht direkt möglich. Sie repräsentieren vielmehr die maximale Produktionspotenz. Cadée \& Hegeman (1974), die nicht nur einen aliquoten Teil, sondern den gesamten Sedimentkern inkubierten, fanden durch die für Algen verbesserten Lichtverhältnisse in den Versuchsgefäßen eine um $60 \%$ erhöhte Produktion gegenüber der ungestörten Sedimentoberfläche. Die Werte aus den Messungen bei Kampen dürften wegen des geringeren Sedimentanteiles in den Inkubationsgefäßen daher gegenüber den aktuellen in situ-Werten noch stärker erhöht sein. Es kam aber für die Fragestellung der vorliegenden Untersuchungen nicht so sehr auf die absoluten Produktionswerte an als vielmehr auf die graduellen Unterschiede im Verschmutzungsgradienten.

Trotz der bisher noch mit Unsicherheiten belasteten Methodik der Produktionsmessungen im Sediment - inhomogene Durchmischungen des Sedimentes, Aktivitätsverluste an ${ }^{14} \mathrm{C}$ durch Zugabe von Formol (Lehmusluoto \& Niemi, 1977), unterschiedliche Lichtverhältnisse während der Inkubation - sind die Ergebnisse durch den Abwassereinfluß interpretierbar. Sie lassen sich außerdem gut mit denen von Grøntved (1960) vergleichen, dèr mit ähnlicher Methodik Produktionsmessungen durchführte.

Durch eine stärkere Förderung der Respiration gegenüber der Photosynthese können Abwassereinleitungen in marine Okosysteme entscheidende Änderungen der Stoff- und Energiekreisläufe verursachen (O'Sullivan, 1971). Auch im Wattengebiet bei Kampen führt die Abwassereinleitung zu einer weitaus höheren Produktion der heterotrophen als der autotrophen Mikroorganismen in der oberen Sedimentschicht.

Die Produktion der Destruenten wurde dabei auf Grund ihrer $\mathrm{CO}_{2}$-Dunkelfixierungsraten ermittelt. Auch andere Autoren benutzten diese Methode zur Bestimmung der bakteriellen Biosynthese, obwohl noch viele Schwierigkeiten bei der Interpretation der Ergebnisse bestehen (Sorokin, 1958, 1964; Steemann Nielsen, 1960; Grøntved, 1962; Overbeck, 1972). Die gesamte mikrobielle Assimilation von $\mathrm{CO}_{2}$ beruht auf der Aktivität verschiedener Gruppen von Mikroorganismen, die $\mathrm{CO}_{2}$ in unterschiedlichen Größenordnungen aufnehmen (Sorokin, 1965; Romanenko, 1964, 1965). Der Anteil des aufgenommenen $\mathrm{CO}_{2}$ an der Produktion organischer Kohlenstoffverbindungen schwankt zwischen $3 \%$ und $100 \%$ (Sorokin \& Kadota, 1972). Ferner wird noch $\mathrm{CO}_{2}-$ sowohl von autotrophen als auch den heterotrophen Organismen - im Rahmen der "Wood-Werkmann-Reaktion" fixiert, ohne daß ein Kohlenstoffgewinn resultiert (Steemann Nielsen, 1960). Diese Art der $\mathrm{CO}_{2}$-Aufnahme ist hier aber zu vernachlässigen, da sie bei Wasserverschmutzungen durch abbaubare organische Substanzen von der mikrobiellen $\mathrm{CO}_{2}$-Fixie- 
rung stark überlagert wird und somit für die Kalkulation der heterotrophen Produktion keine Rolle spielt.

Für die Umrechnung der $\mathrm{CO}_{2}$-Dunkelfixierungsraten in Kohlenstoff-Produktion werden von Sorokin \& Kadota (1972) Faktoren für unterschiedliche Sedimente angegeben, die Oxydationsgrad und Anteil an organischer Substanz berücksichtigen. $\mathrm{Da}$ dies nur empirisch gewonnene, grobe Mittelwerte sind, stellen die vorliegenden Meßwerte nur größenordnungsmäßig die Produktionsverhältnisse im Einleitungsgebiet dar.

\section{Veränderungen der Makrobenthos- Struktur als Folge von Abwassereinleitungen}

Abwassereinleitungen in Wattengebiete führen nicht zu einer Dezimierung des Artenspektrums im gesamten Einflußbereich. Die gefundenen Artenzahlen erscheinen zwar im Vergleich zu anderen Biotopen als niedrig; es muß aber berücksichtigt werden, daß Wattengebiete - als Teil der Macoma balthica-Biozönose (Remane, 1940) - an sich schon einen extremen Lebensraum darstellen, der sich durch Artenarmut (aber hohe Individuendichten) auszeichnet (Wohlenberg, 1937; Linke, 1939; Dörjes, 1970). Setzt man die bei Sylt und Vancouver ermittelten Artenzahlen in Relation zu der jeweiligen untersuchten Sedimentfläche (= Summe der Oberflächen aller Proben), so zeigt sich ebenfalls, daß die Abwasserbelastung zu keiner Reduktion der Artenvielfalt führt. Die Fauna ist in den Einleitungsgebieten teilweise sogar deutlich artenreicher als in unbelasteten Watten vor der holländischen Küste (Beukema, 1976).

Auf den trockenfallenden Wattflächen kommt es durch den zusätzlichen Eintrag von organischem Restmaterial nicht zu einer qualitativen Veränderung der trophischen Struktur des Sedimentes. Daher ist auch kein Wechsel der a priori vorhandenen Ernährungstypen innerhalb des Makrobenthos zu erwarten. Die Konstanz der Dominanz-Verhältnisse von den unbelasteten Bereichen bis in die stark verschmutzten Zonen hinein bestätigen dies. Dagegen wird im Abwasserpriel, in dem sandige Sedimente vorherrschen, das Nahrungsangebot qualitativ und quantitativ verändert: Das Abwasser induziert im Bereich starker und mittlerer Belastung dichte Algen- und Bakterienrasen auf den Sandkörnern, die von Sediment-Abweidern genutzt werden können. Mit geringer werdendem Abwassereinfluß vermindert sich diese Nahrungsquelle. Die Folge ist eine Strukturveränderung des Makrobenthos. Allerdings läßt sich eine natürliche Zonierung der Fauna zwischen Uferbereich und Niedrigwasserlinie nicht von einer durch das Abwasser verursachten Sukzession des Benthos unterscheiden. Ein Vergleich der Dominanz-Werte von 1970 und 1973 (Tab. 13) zeigt aber, daß der Verschmutzungseinfluß überwiegt, da eine Umstrukturierung des Makrobenthos 1973 bei geringerer Belastung wesentlich eher einsetzte.

Verteilungen der Individuenabundanzen und der Artenvielfalt, wie sie im Abwasserpriel bei Kampen und im Abwasserkanal bei Vancouver in den Zonen unterschiedlich starker Belastungen gefunden wurden - Degradationszone, Abundanzmaximum, BasisWerte, bei kontinuierlichem Anstieg der Artenvielfalt mit abnehmender Belastung - sind für sublitorale Abwassereinleitungen hinreichend bekannt (Leppäkoski, 1968; Tulkki, 1968, 1969; Bagge, 1969; Rosenberg, 1973; SCCWRP, 1973; Anger, 1975b; B. C. Research, 1975; Pearson, 1975). Hier machen sich zwei gegenläufig wirkende, abwasserbeein- 
flußte Okofaktoren geltend: zum einen der chemisch-physikalische Umweltkomplex und zum anderen das Nahrungsangebot. Im unmittelbaren Bereich von Abwassereinleitungen sind die Lebensbedingungen selbst für tolerante Arten trotz eines optimalen Nahrungsangebotes so wenig akzeptabel, daß es hier in der Regel zu Verödungszonen ohne Makrobenthos kommt. Mit der Entfernung von der Einleitungsstelle nimmt der Abwasserbelastungsfaktor (Tab. 2) schnell ab, während das Nahrungsangebot (organische Abwasserpartikel und dichte, vom Abwasser induzierte Algen- und Bakterienrasen) weiterhin erhöht bleibt. Die Belastung wird dann für euryöke Organismen, also für die meisten Wattarten, erträglich, so daß sie das gute Nahrungsangebot voll nutzen können (s. a. Oliff et al., 1967, und O'Sullivan, 1971). Da das Artenspektrum überwiegend auf euryöke Formen begrenzt bleibt, erreichen diese hohe Individuendichten; ferner entfällt die Nahrungskonkurrenz sensiblerer Arten. Im Anschluß an diese Zone höchster Abundanzen nimmt die Verschmutzung und damit die Zufuhr organischen Restmaterials ab, wodurch die Zahl der ökologischen Nischen ansteigt (sog. "edge effect"). Es können zunehmend empfindlichere Arten das Sediment besiedeln, ohne daß die abwassertolerierenden Formen verdrängt werden. Zusätzlich zum verminderten Nahrungsangebot führt dies zu einer Verschärfung der Nahrungskonkurrenz, wodurch Massenvorkommen einzelner Arten unterbunden werden. Die Verteilung des Benthos im Priel erfüllt damit das zweite biozönotische Gesetz von Thienemann (1941).

Dagegen entsprechen auf den Wattflächen die Verteilungsmuster der Arten und Individuen in den Zonen unterschiedlicher Belastung nicht diesem Prinzip. Die Ursache wird in den unterschiedlichen Umweltverhältnissen gesehen: Schlickwatten repräsentieren im Gegensatz zum Wattenpriel, wegen des periodischen Trockenfallens und aller damit zusammenhängenden Streßfaktoren, einen Extrembiotop, der nur von wenigen euryöken Arten besiedelt werden kann, allerdings mit der Folge, daß das hohe Nahrungsangebot in Form von sedimentiertem Detritus die Entwicklung großer Individuenzahlen ermöglicht. Durch den zusätzlichen Import von organischem Restmaterial aus dem Abwasser kommt es zu einer weiteren Erhöhung der Siedlungsdichten, die aber irn Gegensatz zum Priel mit einer Steigerung der Artenvielfalt verbunden ist. Es ist zu vermuten, daß die Nahrungskonkurrenz zwischen den euryöken Arten durch ein Überangebot an Nahrung vermindert wird und sich deshalb zusätzlich weitere, abwassertolerante, aber sonst ökologisch schwächere Wattenarten ansiedeln können. Wird der Abwassereinfluß zu groß, finden auch die tolerantesten Formen keine Existenzmöglichkeit mehr; die Folge ist eine Degradationszone im Verschmutzungszentrum.

Eine weitere Ursache für die verschiedenartige Reaktion der Priel- und Wattfauna wird darin gesehen, daß auf den Wattflächen ein direkter Abwasserkontakt nicht gegeben ist: Während bei Niedrigwasser das Prielsediment, und bei auf- und ablaufender Tide auch kurzzeitig die unmittelbar benachbarten Flächen, dem Abwasser ausgesetzt sind, beschränkt sich für die Wattensedimente der Abwassereinfluß auf ein Sedimentieren partikulärer Substanzen. Die Fauna braucht sich dort folglich nur mit den verstärkten Abbauvorgängen bei der Dekomposition der festen Abwasserbestandteile auseinanderzusetzen. $\mathrm{Da}$ das organische Material nur in Gebieten abgelagert wird, die durch die Strömungsverhältnisse schon einer natürlichen Aufschlickung unterliegen, setzt sich das hier angesiedelte Makrobenthos a priori aus Arten zusammen, die bereits an Mineralisations- und Fäulnisvorgänge im Sediment adaptiert sind. Als Indikatoren für organische 
Abwassereinleitungen in Schlickwatten sind sie daher nicht geeignet. Es liegt vielmehr nahe, die Schlickwatten-Arten daraufhin zu testen, bis zu welchem Grad sie eine zusätzliche Saprobitätserhöhung in ihrem Biotop ertragen können, ohne akute oder chronische Schädigungen davonzutragen. Auf Grund solcher Tests wird es erst möglich sein, die Auswirkungen einer geplanten Abwassereinleitung in schlickige Wattengebiete abschätzen zu können. Einen Hinweis auf Grenzwerte geben die Analysen der sedimentären organischen Substanz aus dem Untersuchungsgebiet Vancouver: Bereiche, in denen ihr Anteil im Sediment über $4 \%$ beträgt, zeichnen sich durch eine drastische Reduktion der Arten- und Individuenzahlen aus; bei Werten von über $8 \%$ bleibt der Wattenboden nahezu unbesiedelt.

Die Diversitätsindices (zur Diskussion über Diversitätsindices siehe Eberhard, 1969; Hurlbert, 1971; Hill, 1973) eignen sich gut, um den Einfluß des Abwassers auf das Makrobenthos zu zeigen. Es kann aber aus den Maximalwerten im jeweiligen Transekt nicht notwendigerweise auf optimale Umweltverhältnisse für die Benthos-Assoziationen geschlossen werden. Odum (1975) wies darauf hin, daß bei der Benutzung der Diversität als Index für Verschmutzung oder sonstige anthropogenen Eingriffe in Biotope Vorsicht geboten ist: Bei vorhandener hoher Diversität wird die biotische Mannigfaltigkeit durch Streß-Erhöhung, wie z. B. Abwassereinleitungen, in der Regel herabgesetzt (Wilhm, 1967; Bechtel \& Copeland, 1970; Boesch, 1972; B. C. Research, 1975). Hat ein System aber von vornherein eine geringe Diversität, wie es für die Watten zutrifft, so ist es möglich, daß durch anthropogene Einflüsse die Diversitätsrate sogar ansteigt. Dies trifft besonders bei einem Input von Energie, z. B. durch Abwassereinleitungen, zu (s. a. Barret, 1969).

Eine intensivere Vorreinigung des eingeleiteten Abwassers führt in den Watten zur Einschränkung oder völligen Vermeidung einer Degradationszone sowie zu einer höheren Diversität und zu einem Wechsel in der Makrobenthos-Struktur. Die Zusammensetzung der Fauna zeigt dabei eine Tendenz zu empfindlicheren Arten und geringeren Individuendichten. Solche Veränderungen weisen in der Regel auf eine Abnahme der Umweltbelastung hin.

Erstaunlicherweise reagiert das Benthos der trockenfallenden Wattenflächen genau konträr auf die Einleitung geringer belasteten Abwassers: Die wenigen dominanten Arten treten durch gesteigerte Individuenzahlen noch weiter gegenüber den individuenärmeren in den Vordergrund. Es ist zu vermuten, daß diese Reaktion durch eine verschärfte Konkurrenz um das (pro Flächeneinheit) verminderte Detritusangebot aus dem Abwasser bedingt ist. Euryöke und resistente Arten können wegen der geringeren Belastung in das Verschmutzungszentrum vordringen und diesen Raum, bei fehlender Konkurrenz, mit hohen Individuendichten besiedeln.

Erstaunlich ist das fast völlige Verschwinden von Corophium volutator. Dieser Amphipode besiedelte 1970 noch mit hohen Individuendichten die Wattflächen im Einleitungsgebiet von Kampen/Sylt (Otte, 1977). Den Grund in der veränderten Abwasserqualität zu suchen, scheint nicht zulässig, da er zu den resistenten Formen gezählt werden muß (Fraser, 1932; Clay, 1969). Auch ein vermindertes Detritusangebot kann als Ursache ausgeschlossen werden, weil er 1975 wieder mit hohen Individuenzahlen den gleichen Wattenbereich besiedelte. Wahrscheinlicher ist ein starker Befall mit Trematoden-Larven, wie ihn Ankel (1962) und Muus (1967) in dänischen Gewässern beobachteten. Beide Autoren stellten fest, daß die Hydrobien, besonders in flachen Küstengewässern, äußerst 
stark mit Cercarien befallen sind. Diese Parasitierung stellt nicht so sehr eine Gefahr für die Mollusken dar als vielmehr für die im gleichen Gebiet vorkommenden Crustaceen. So konnte Muus (1967) in der Beltsee (Nivå Bay) ein vollständiges Auslöschen der lokalen Corophium-Bestände durch die Cercarien beobachten, wobei die Parasiten von den Hydrobien stammten. Dies ist für das Wattengebiet bei Kampen auch wahrscheinlich, zumal Hydrobia ulvae in großen Dichten - besonders in der von Corophium volutator bevorzugten Zone - gefunden wurde. Außerdem sind die Mollusken im Sylter Watt, vornehmlich im Einflußbereich der Kläranlagen, stark mit Cercarien befallen (Lauckner, 1971; sowie mündliche Mitteilung von Dr. Lauckner, List/Sylt).

Untersuchungen der Fauna-Sediment-Beziehungen zeigen, daß abwasserbeeinflußte Wattengebiete überwiegend von eurytopen Schlicksandarten besiedelt werden. Eine typische, für Detritus stenöke Faunen-Assoziation ist nicht entwickelt. Alle Elemente der Schlicksand-Fauna wandern vielmehr auch in die Schlickbereiche ein, soweit die abiotischen Okofaktoren es noch erlauben. In sandige Sedimente dringen dagegen nur wenige Arten dieser Faunen-Gruppen ein.

Johnson (1970) kam bei ähnlichen Untersuchungen im marinen Sublitoral zu teilweise gegensätzlichen Ergebnissen. Er konnte nachweisen, daß in seinem Gebiet die SchlicksandArten zahlreicher im Sand als im Schlick vorkamen. Es ist anzunehmen, daß dies konträre Verhalten für die Wattenfauna charakteristisch ist. Die hohe Stabilität des "Okosystem Watt" gegenüber organischen Abwassereinleitungen könnte zum Teil durch diese Adaptionsfähigkeit des Makrobenthos für Schlick-Sedimente begründet sein.

Abschließend sei darauf hingewiesen, daß sich aus den übereinstimmenden Ergebnissen der Paralleluntersuchungen in marinen Nordsee-Watten (Sylt) und in aestuarinen pazifischen Watten (Vancouver) allgemeine Gesetzmäßigkeiten für die biologischen Auswirkungen von kommunalen Abwassereinleitungen gewinnen ließen. Dies erscheint jedoch bei ausschließlicher Verwendung des vorliegenden Datenmaterials noch zu unsicher. Nur mehrjährige Dauermessungen würden zu statistisch absicherbaren Aussagen über Veränderungen biotischer und abiotischer Faktoren führen, die die natürlichen Fluktuationen in diesem Lebensraum überlagern.

Die vorliegenden Beobachtungen beziehen sich auf ein vom Menschen vorgenommenes Großexperiment: die Auswirkung von biologisch oder nur mechanisch gereinigten Abwässern auf Wattenflächen. Die Frage, ob dieses technische Vorgehen vom Gesichtspunkt der Erhaltung eines funktionierenden Okosystems zu vertreten ist, muß jeweils nach den lokalen Gesichtspunkten diskutiert werden. Für die untersuchten Gebiete bei Sylt und Vancouver ist zumindest anzugeben, $\mathrm{da} ß$ in den Wattenbereichen keine totale Dezimierung des vorhandenen benthischen Okosystems eintrat. Lokal abgegrenzte Schädigungszonen im Einleitungszentrum ließen sich durch eine vollbiologische Vorreinigung des Abwassers weitgehend vermeiden. Es wurde herausgestellt, daß die "Potenz" solcher Gebiete für die Dekomposition zusätzlicher Mengen abbaubarer organischer Reststoffe erstaunlich hoch ist.

Danksagungen. Herrn Professor Dr. H. Caspers danke ich für die Uberlassung eines Arbeitsplatzes im Institut für Hydrobiologie und Fischereiwissenschaften der Universität Hamburg. Ihm danke ich auch, ebenso wie Herrn Dr. H. Rosenthal von der Biologischen Anstalt Helgoland, für die kritische Durchsicht des Manuskriptes. Die Untersuchungen auf Sylt wurden in der Litoralstation der Biologischen Anstalt Helgoland und die Untersuchungen in Vancouver/Canada im Pacific Environ- 
ment Institute durchgeführt. Für die in beiden Instituten gewährte Unterstützung danke ich Herrn Professor Dr. O. Kinne, Herrn Dr. E. Ziegelmeier sowie Herrn Dr. M. Waldichuk und Herrn Dr. C. Levings. Frl. J. Willführ fertigte für mich den größten Teil der Zeichnungen an. Ein Teil der Arbeiten wurde mit Mitteln der Deutschen Forschungsgemeinschaft durchgeführt. Den Aufenthalt in Canada finanzierte die Gesellschaft für Kernenergieverwertung in Schiffbau und Schiffahrt (GKSS), Geesthacht, im Rahmen eines wissenschaftlich-technologischen Austauschprogrammes zwischen der Bundesrepublik Deutschland und Canada.

\section{ZITIERTE LITERATUR}

Akeson, B., 1970. Opbryotrocha labronica as a test animal for the study of marine pollution. Helgoländer wiss. Meeresunters. 20, 293-303.

Anderson, K. J. \& Read, P. A., 1974. Pollution studies in the Firth of Forth. Mar. Pollut. Bull. 5, $121-125$.

Anderson, S. S., 1972. The ecology of Morecambe Bay. II. Intertidal invertebrates and factors affecting their distribution. J. appl. Ecol. 9, 161-178.

Anger, K. 1975 a. Benthos und Abwasser. Die Auswirkung kommunaler Abwässer auf ein ufernahes Partialökosystem der westlichen Ostsee. Diss., Univ, Kiel, 327 pp.

- $1975 \mathrm{~b}$. On the influence of sewage pollution on inshore benthic communities in the South of Kiel Bay. 2. Quantitative studies on community structure. Helgoländer wiss. Meeresunters. 27, 408-438.

- 1977. Benthic invertebrates as indicators of organic pollution in the western Baltic Sea. Int. Revue ges. Hydrobiol. 62, 245-254.

Ankel, F., 1962. Hydrobia ulvae und Hydrobia ventrosa als Wirte larvaler Trematoden. Vidensk. Meddr. dansk naturh. Foren. 124, 1-100.

Bagge, P., 1969. Effects of pollution on estuarine ecosystems. I. and II. Merentutkimuslait. Julk. 228, $1-129$.

Balogh, H., 1958. Lebensgemeinschaften der Landtiere. Akademie Verlag, Berlin, 500 pp.

Banse, K. \& Hobson, K. D., 1974. Benthic errantiate polychaetes of British Columbia and Washington. Bull. Fish. Res. Bd Can. 85, 1-111.

Barnard, J. L., 1959. Estuarine amphipods. Part II. Occ. Pap. Allan Hancock Fdn 21, 13-69.

- 1969. The families and genera of marine gammaridean amphipoda. Bull. U. S. nat. Mus. 271, $1-525$.

Barrett, G. W. P., 1969. The effects of an acute insecticide stress on a semi-enclosed grassland ecosystem. Ecology 49, 1019-1035.

B. C. Research, 1974. Environmental studies at Iona Island. Rep. B. C. Research, Vancouver, Canada, Div. Appl. Biol. 1, 1-55.

- 1975. Environmental studies at Iona Island. Rep. B. C. Research', Vancouver, Canada, Div. Appl. Biol. 2, 1-162.

Bechtel, T. J. \& Copeland, B. J., 1970. Fish species diversity indices as indicators of pollution in Galveston Bay, Texas. Contr. mar. Sci. 15, 103-132.

Berkeley, E. \& Berkeley, C., 1952. Annelida, Polychaeta Errantia. Can. Pacif. Fauna 96, 1-100.

Beukema, J. J., 1974. Seasonal changes in the biomass of the macrobenthos of a tidal flat area in the Dutch Wadden Sea. Neth. J. Sea Res. 8, 94-107.

- 1976. Biomass and species richness of the macrobenthic animals living on the tidal flats of the Dutch Wadden Sea. Neth. J. Sea Res. 10, 236-261.

Beyer, F., 1968. Zooplankton, zoobenthos, and bottom sediments as related to pollution and water exchange in the Oslofjord. Helgoländer wiss. Meeresunters. 17, 496-509.

Bianchi, A. J. M., 1973. Variations de la concentration bacteriénne dans les eaux et les sédiments littoraux. Mar. Biol. 22, 23-29.

Blegvad, H., 1932. Investigations of the bottom fauna of drains in the Sound. Rep. Dan. biol. Stn 37, $1-20$. 
Bloom, S. A., Simon, J. L. \& Hunter, V. D., 1972. Animal-sediment relations and community analysis of a Florida estuary. Mar. Biol. 13, 43-56.

Boesch, D. F., 1972. Species diversity of marine macrobenthos in the Virginia area. Chesapeake Sci. 13, 206-211.

Borowitzka, M. A., 1972. Intertidal algal species diversity and effect of pollution. Aust. J. mar. Freshwat. Res. 23, 73-84.

Brinkhurst, R. O., 1966. The use of aquatic oligochaeta worms in the detection assessment of water pollution. World Health Organisation/ EBL/62, 70, 1-20.

Buchmann, J. B. \& Longbottom, M. R., 1970. The determination of organic matter in marine muds: the effect of the presence of coal and of the routine determination of protein. J. exp. Biol. Ecol. 5, 158-169.

Burkholder, P. A., Repark, A. \& Siebert, G., 1965. Studies on some Long Sound littoral communities of microorganisms and their primary productivity. Bull. Torrey bot. Club 92, 378-402.

Cadée, G. C. \& Hegeman, J., 1974. Primary production of the benthic microflora living on tidal flats in the Dutch Wadden Sea. Neth. J. Sea Res. 8, 260-291.

Caspers, H., 1969. Der Einfluß der Elbe auf die Verunreinigung der Nordsee. Helgoländer wiss. Meeresunters. 17, 422-434.

Cavalli-Sforza, L., 1968. Biometrie. Fischer, Stuttgart, 211 pp.

Clay, E., 1967 a. Literature survey of the common fauna of estuaries. 3. Pygospio elegans. I. C. I. Paints Div., Marine Research Station, Brixham, Devon, 1-12. (Mimeogr.).

- 1967 b. Literature survey of the common fauna of estuaries. 15. Corophium volutator. I. C. I. Paints Div., Marine Research Station, Brixham, Devon, 1-21. (Mimeogr.).

Coastal Water Research Project, 1977. Southern California Coastal Water Research Project. A. Rep. 1977, 1-253.

Crawford, G. I., 1937. A review of the amphipod genus Corophium, with notes on the British species. J. mar. biol. Ass. U. K. 21, 589-630.

Croft, J. E., 1960. Pollution of coastal and estuarial waters. J. Proc. Inst. Sew. Purif. 4, 431-438.

Dale, N. G., 1974. Bacteria in intertidal sediments: Factors related to their distribution. Limnol. Oceanogr. 19, 509-518.

Day, J. H., 1967. A monograph on the Polychaeta of Southern Africa. Part I, II. Publs Br. Mus. nat. Hist. 656, 1-378.

Dörjes, J., 1970. Das Watt als Lebensraum. In: Das Watt. Hrsg. von H. Reineck, Kramer, Frankfurt, 71-105.

Downing, A. L. \& Wheatland, A. B., 1962. Fundamental considerations in biological treatment of effluents. Trans. Instn chem. Engrs 40, 91 pp.

Duedall, J. W., Bowman, M. J. \& O'Connors jr., H. B., 1975. Sewage sludge and ammonium concentrations in the New York Bight apex. Estuar. coast. mar. Sci. 3, 457-463.

Duff, S. \& Teal, J. M., 1965. Temperature change and gas exchange in Nova Scotia and Georgia saltmarsh muds. Limnol. Oceanogr. 10, 67-73.

Eberhardt, L. L., 1969. Some aspects of species diversity models. Ecology 50, 503-505.

Edwards, R. W. \& Rolley, H. L. J., 1965. Oxygen consumption of river muds. J. Ecol. 53, 1-19.

Essink, K., 1972. Onderzock naar de gevolgen van lozing van ongezuiverd industrieel afvalwater door middel van een persleiding van Hoogkerk naar de Waddenzee. Interimrapport over 1971. Zoölogisch Laboratorium der Rejksuniversiteit te Groningen Z 72/ 122, 1-24.

Fauvel, P., 1923. Polychètes errantes. Faune Fr. 5, 1-488.

- 1927. Polychètes sédentaires. Faune Fr. 16, 1-494.

Felice, F. P., 1959. The effect of wastes on the distribution of bottom invertebrates in the San Francisco Bay estuary. Wasmann J, 17, 1-70.

Fenchel, T. \& Staarup, B. J., 1971. Vertical distribution of photosynthetic pigments and the penetration of light in marine sediments. Oikos 22, 172-182.

Folk, R. L., 1966. A review of grain-size parameters. Sedimentology 6, 73-93.

Frankenberg, D. \& Westerfield, C. W., 1968. Oxygen demand and oxygen depletion capacity of sediments from Wassaw Sound, Georgia. Bull. Ga. Acad. Sci. 26, 160-172.

Fraser, J. H., 1932. Observations of the fauna and constituents of an estuarine mud in a polluted area. J. mar. biol. Ass. U. K. 18, 69-85. 
Friedrich, H., 1938. Polychaeta. Tierwelt Nord- u. Ostsee. 6 b, 1-201.

- 1965. Meeresbiologie. Borntraeger, Berlin, 436 pp.

Gadow, S., 1970. Sedimente und Chemismus. In: Das Watt. Hrsg. von H. Reineck. Kramer, Frankfurt, 23-35.

Gargas, E., 1971. "Sun-shade" adaptation in microbenthic algae from the Øresund. Ophelia 9, $107-112$.

- 1972. Measurements of microalgal primary production (phytoplankton and microbenthos) in the Smalandshavet (Denmark). Ophelia 10, 75-89.

Georgia water quality control board. 1967. Establishment of water quality standard and classifications for interstate waters in the State of Georgia. Georgia Water Quality Control Board, Atlanta, 145 pp. (Mimeogr.)

Gray, J. S., 1974. Animal-sediment relationships. Oceanogr. mar. Biol. 12, 223-261.

Grøntved, J., 1960. On the productivity of microbenthos and phytoplankton in some Danish fjords. Meddr Kommn Danm. Fisk.-og Havunders. 3, 55-92.

-1962. Preliminary report on the productivity of microbenthos and phytoplankton in the Danish Wadden Sea. Meddr Kommn Danm. Fisk.-og. Havunders. 3, 347-378.

Gross, M. G., 1972. Marine waste deposits near New York. Mar. Pollut. Bull. 3, 61-63.

Hargrave, B. T., 1972. Aerobic decomposition of sediment and detritus as a function of particle surface area and organic content. Limnol. Oceanogr. 17, 583-596.

Hartmann-Schröder, G., 1971. Annelida, Borstenwürmer, Polychaeta. Tierwelt Dtl. 58, 1-594.

Hauser, B., 1973. Bestandsänderungen der Makrofauna an einer Station im ostfriesischen Watt. Jber., ForschSt. Norderney 24, 171-203.

Hecht, F., 1933. Der Verbleib der organischen Substanz der Tiere bei meerischer Einbettung. Senkenbergiana 15, 165-249.

Hill, M. O., 1973. Diversity and eveness: a unifying notation and its consequences. Ecology 54, $427-432$.

Hoos, L. M. \& Packman, G. A., 1974. The Fraser River estuary. Status of environmental knowledge to 1974. Environment Canada. Spec. Estuary Ser. 1, 1-518.

Hopkins, J. T., 1963. A study of the diatoms of the Ouse estuary, Sussex. The movement of the mudflat diatoms in response to some chemical and physical changes. J. mar. biol. Ass. U. K. 43, 653-663.

Hughes, R. N., 1970. An energy budget for a tidal flat population of the bivalve Scrobicularia plana. J. Anim. Ecol. 39, 357-381.

Hurlebert, S. H., 1969. A coefficient of interspecific association. Ecology 50, 1-9.

Jacobs, J., 1975. Diversity, stability, and maturity in ecosystems influenced by human activities. In: Unifying concepts in ecology. Ed. by W. H. van Dobben \& R. H. Lowe-McConnell. Junk, The Hague, 187-207.

Jarvekülg, A. A., 1970. Bottom fauna as an indicator of pollution of the marine benthal in the vicinity of Tallinn. Est. Contr. int. biol. Progr. Acad. Sci. Estn. SSR, 1, 158-193.

Jenkinson, J. R., 1972. Sludge dumping and benthic communities. Mar. Pollut. Bull. 3, 102-105.

Johnson, R. G., 1971. Animal-sediment relations in shallow water benthic communities. Mar. Geol. 11, 93-104.

Johnson, W. K. \& Schroepfer, G. J., 1964. Nitrogen removal by nitrification and denitrification. J. Wat. Pollut. Control Fed. 36, 1015-1036.

Jones, N. S. \& Sanders, H. L., 1972. Distribution of Cumacea in the deep Atlantic. Deep Sea Res. 19, 737-745.

DeJonge, V. N. \& Postma, H., 1974. Phosphorus compounds in the Dutch Wadden Sea. Neth. J. Sea Res. 8, 139-153.

Knowles, G. et al., 1962. Polarographic measurement of the rate of respiration of natural sediments. Limnol. Oceanogr. 7, 481.

König, D., 1968. Biologische Auswirkung des Abwassers einer Ol-Raffinerie in einem Vorlandgebiet an der Nordsee. Helgoländer wiss. Meeresunters. 17, 321-334.

Köster, E., 1964. Granulometrische und morphometrische Meßmethoden an Mineralkörnern, Steinen und sonstigen Stoffen. Enke, Stuttgart, 336 pp. 
Kolkwitz, R. \& Marsson, M., 1908. Okologie der pflanzlichen Saprobien. Ber. dt. bot. Ges. 26, 126-159.

- 1909. Okologie der tierischen Saprobien. Int. Revue ges. Hydrobiol. 2, 126-152.

Kolt, J. \& Ben Ari, 1968. Bacteriophages as marine pollution indicators. Revue int. Océanogr. méd. 9, 203-217.

Krumbein, W. E., 1971. Sedimentmikrobiologische Untersuchungen. I. Uber die Abhängigkeit der Keimzahl von der Korngröße. Vie Milieu (Suppl.) 22, 253-264.

Lauckner, G., 1971. Zur Trematodenfauna der Herzmuscheln Cardium edule und Cardium lamarcki. Helgoländer wiss. Meeresunters. 22, 377-400.

Leach, J. H., 1970. Epibenthic algal production in an intertidal mudflat, Limnol. Oceanogr. 15, 514-521.

Lehmusluota, P. O. \& Niemi, A., 1977. Effect of fixation with formalin and Lugol's solution on ${ }^{14} \mathrm{C}$ measurements of phytoplankton production. Merentutkimuslait. Julk. 241, 97-100.

Leppäkoski, E., 1968. Some effects of pollution on the benthic environment of the Gullmarsfjord. Helgoländer wiss. Meeresunters. 17, 291-301.

- 1975. Assessment of degree of pollution on the basis of macrozoobenthos in marine and brackishwater environments. Acta Acad. åbo. (B) 25, 1-90.

Levings, C. D. \& Coustalin, J. B., 1975. Zonation of intertidal biomass and related benthic data from Sturgeon and Roberts Banks, Fraser River Estuary, British Columbia. Tech.Rep. Fish.Mar. Serv. $468,1-138$.

Liebmann, H., 1947. Die Notwendigkeit einer Revision des Saprobiensystems und deren Bedeutung für die Wasserbeurteilung. Gesundheitsingenieur 68, 33-37.

- 1960, 1962. Handbuch der Frischwasser- und Abwasserbiologie. Oldenbourg, München, 1-2; 588, 1149 pp.

Lillelund, K., 1969. Die Bedeutung von Corophium für die Rückführung von $P$ aus dem Sediment in das freie Wasser. In: Bericht über das Kolloquium "Litoralforschung/Abwasser in Küstennähe". Deutsche Forsch.Gemeinsch., Bonn, 42-44. (Mimeogr.)

Linke, O., 1939. Die Biota des Jadebusenwattes. Helgoländer wiss. Meeresunters. 1, 201-348.

Longbottom, M. R., 1970. The distribution of Arenicola marina with particular reference to the effects of particle size and organic matter of the sediments. J. exp. mar. Biol. Ecol. 5, 138-157.

Ludwig, H. F. \& Storrs, P. N., 1970. Effects of waste disposal into marine waters. A survey of studies carried out in the last ten years. Wat. Res. 4, 709-720.

Martin, D. C. \& Bella, D. A., 1971. Effect of mixing on oxygen uptake rate of estuarine bottom deposits. J. Wat. Pollut. Control Fed. 43, 1865-1876.

McArthur, R. H. \& McArthur, J. W., 1961. On bird species diversity. Ecology 42, 594-598.

McMillan, G. L. \& Verduin, C., 1953. Photosynthesis of natural communities dominated by Cladopbora glomerata und Ulothrix zonata. Ohio J. Sci, 53, 373-377.

McNulty, J. K., Work, R. C. \& Moor, H. B., 1962. Some relationships between the infauna of the level bottom and the sediments of southern Florida. Bull. mar. Sci. Gulf Caribb. 12, 322-332.

Moul, E. T. \& Mason, D., 1957. Study of diatom populations on sand and mud flats in the Woods Hole area. Biol. Bull. mar. biol. Lab., Woods Hole, 113, 351.

Muus, B. J., 1967. The fauna of Danish estuaries and lagoons. Meddr Danm. Fisk.-og. Havunders. 5, 5-316.

Nakaïma, F., 1958. Plankton as indicators of sea water pollution. Bull. Inst. publ. Hlth, Tokyo 7, $140-145$.

National Technical Advisory Committee to the Secretary of the Interior, 1968. Water quality criteria. U. S. Government Printing Office, Washington, $234 \mathrm{pp}$.

Newell, R. C., 1970. Biology of intertidal animals, Logos Press, London, 555 pp.

Nichols, M. M., 1964. Characteristics of sedimentary environment in Moriches Bay, N. Y. In: Papers in marine geology. Ed. by R. L. Miller. McMillan, New York.

O'Connor, J. S., 1972. The benthic macrofanna of Moriches Bay, N. Y. Biol. Bull. mar. biol. Lab., Woods Hole. 142, 84-102.

Odum, E. P., 1975. Diversity as a function of energy flow. In: Unifying concepts in ecology. Ed. by W. H. Dobben \& R. H. Lowe-McConnell. Junk, The Hague, 11-14. 
Odum, H. T., 1957. Primary production measurements in eleven Florida springs and a marine turtlegrass community. Limnol. Oceanogr. 2, 85-97.

Oglesby, R. T. \& Jamison, D., 1968. Intertidal communities as monitors of pollution. J. sanit. Engng Div. Am. Soc. civ. Engng 94, 541-550.

Oliff, W. D., Berrisford, C. D., Turner, W. D., Ballard, J. A. \& McWilliams, D. C., 1967. The ecology and chemistry of sandy beaches and nearshore submarine sediments of Natal. I. Pollution criteria for sandy beaches in Natal. II. Pollution criteria for nearshore sediments of the Natal coast. Wat. Res. 1, 115-129, 131-146.

Olsen, T. A. \& Burgess, F. J. (Ed.), 1967. Pollution and marine ecology. Wiley Interscience, London, $364 \mathrm{pp}$.

O'Sullivan, A. J., 1971. Ecological effects of sewage discharge in the marine environment. Proc. R. Soc. (B), 177, 331-351.

Otte, G., 1975. A laboratory key for the identification of Corophium species (Amphipoda, Corophiidae) from British Columbia. Tech. Rep. Fish. Mar. Serv. 519, 1-28.

- 1977. Organische Abwassereinleitungen in Wattengebiete: Versuch einer saprobiellen Wertung. Ergebn. Limnol. 9, 139-150.

Overbeck, J., 1972. Experimentelle Untersuchungen zur Bestimmung der bakteriellen Produktion im See. Verh. int. Verein. theor. angew. Limnol. 18, 176-187.

Pamatmat, M. M., 1968. Ecology and metabolism of a benthic community on an intertidal sandflat. Int. Revue ges. Hydrobiol. 53, 211-298.

- 1971. Oxygen consumption by the seabed. IV. Shipboard and laboratory experiments. Limnol. Oceanogr. 16, 536-550.

Patterson, J. \& Parsons, T. R., 1963. Distribution of chlorophyll a and degradation products in various marine materials. Limnol. Oceanogr. 8, 355-356.

Pearson, T. H., 1975. The benthic ecology of Loch Linnhe and Loch Eil, a sea-loch system on the west coast of Scotland. IV. Changes in the benthic fauna attributable to organic enrichment. J. exp. mar. Biol. Ecol. 20, 1-41.

Perkins, E. J. \& Abbott, O. J., 1972. Nutrient enrichment and sand flat fauna. Mar. Pollut. Bull. 3, 70-72.

Pestrong, R., 1972. Tidal-flat sedimentation at Cooley Landing, Southwest San Francisco Bay. Sediment. Geol. 8, 251-288.

Postma, H., 1954. Hydrography of the Dutch Wadden Sea. Archs neerl. Zool. 10, 405-511.

- 1960. Einige Bemerkungen über den Sinkstofftransport im Ems-Dollart-Gebiet. Verh. K. ned. geol.-mijnb. Genoot. 19, 103-110.

— \& Kalle, K., 1955. Die Entstehung von Trübungszonen im Unterlauf von Flüssen, speziell im Hinblick auf die Verhältnisse in der Unterelbe. Dt. hydrogr. Z. 8, 137-144.

Quayle, D. B., 1960. The intertidal bivalves of British Columbia. B. C. Prov. Mus. Handb. 17, 1-117.

Reineck, H. E. (Ed.), 1970. Das Watt, Kramer, Frankfurt, 142 pp.

Reish, D. J., 1955. The relation of the polychaetous annelids to harbor pollution. U. S. Pub. Health Rep. 70, 1168-1174.

- 1957. Effects of pollution on marine life. Ind. Wastes 2, 114-118.

- 1959. An ecological study of pollution in Los Angeles-Long Beach Harbors, Calif. Occ. Pap. Allan Hancock Fdn 22, 1-119.

- 1960. The use of marine invertebrates as indicators of water quality. In: Proceedings of the $1 s t$ international conference on waste disposal in the marine environment. Ed. by E. A. Pearson., New York, 92-103.

- 1970. A critical review of the use of marine invertebrates as indicators of varying degrees of marine pollution. FAO techn. conf. on mar. pollut. and its effects on living resources and fishing, Rome, Italy, FIR: MP/70/R - 9 .

- 1972. The use of marine invertebrates as indicators of varying degrees of marine pollution. In: Marine pollution and sea life. Ed. by M. Ruivo. Fishing News Books, London, 203-207.

Remane, A., 1940. Einführung in die zoologische Okologie der Nord- und Ostsee. Tierwelt Nord- u. Ostsee. 1 a, 1-238.

Rhoads, D. C. \& Young, D. K., 1970. The influence of deposit-feeding organisms on sediment stability and community trophic structure. J. mar. Res. 28, 150-177. 
Rice, T., 1972. Marine shells of the Pacific Northwest. Bradley, Seattle, Wash., 102 pp.

Romanenko, W. I., 1964. Heterotrophic assimilation of $\mathrm{CO}_{2}$ by the aquatic microflora. Microbiologia 33, 679-683.

- 1964. The potential ability of the bottom microflora to the hererotrophic assimilation of $\mathrm{CO}_{2}$ and to the chemosynthesis. Microbiologia 33, 134-139.

- 1965. The relation between the consumption of oxygen and $\mathrm{CO}_{2}$ by heterotrophic bacteria during the growth in the presence of peptone. Microbiologia 34, 391-396.

Rosenberg, R., 1971. Recovery of the littoral fauna in Saltstkällefjord subsequent to discontinued operations of a sulphite pulp mill. Thalassia jugosl. 7, 341-351.

- 1973. Succession in benthic marcrofauna in a Swedish fjord subsequent to the closure of a sulphite pulp mill. Oikos 24, 244-258.

Sachs, L., 1971. Statistische Auswertungsmethoden. Springer, Berlin, 545 pp.

Sanders, H. L., 1958. Benthic studies in Buzzard's Bay. I. Animal-sediment relationships. Limnol. Oceanogr. 3, 245-258.

- 1968. Marine benthic diversity: a comparative study. Am. Nat. 102, 243-282.

SCCWRP, 1973. The ecology of the Southern California Bight: Implications for water quality management. Southern California Coastal Water Research Project. El Segundo, California, $531 \mathrm{pp}$.

Schellenberg, A., 1942. Krebstiere oder Crustacea. IV. Flohkrebse oder Amphipoda. Tierwelt Dtl. 40, $1-242$.

Shannon, C. E. \& Weaver, W., 1949. The mathematical theory of communication. Univ. Ill. Press, Urbana, $117 \mathrm{pp}$.

Shelton, R. G. J., 1971. Sludge dumping in the Thames estuary. Mar. Pollut. Bull. 2, 24-27.

Shoemaker, C. R., 1934 a. The amphipod genus Corophium on the east coast of America. Proc. biol. Soc. Wash. 47, 23-32.

- 1934 b. Two new species of Corophium from the west coast of America. J. Wash. Acad. Sci. 24, 356-360.

- 1941. A new genus and a new species of amphipoda from the pacific coast of North America. Proc. biol. Soc. Wash. 54, 183-186.

- 1949. The amphipod genus Corophium on the west coast of America. J. Wash. Acad. Sci. 39, 66-82.

Sorokin, $\vec{Y}$. I.,, 1958. Studies of chemosynthesis in bottom sediments with the use of ${ }^{14} \mathrm{C}$. Microbiologia 27, 206-213.

- 1964. On the trophic role of chemosynthesis in water bodies. Int. Revue ges. Hydrobiol. 49, 307-324.

- 1965. On the trophic role of chemosynthesis and bacterial biosynthesis in water bodies. Memorie Ist. ital. Idrobiol. 18, 187-205.

- \& Kadota, H. (Ed.), 1972. Techniques for the assessment of microbial production and decomposition in fresh waters. Blackwell, Oxford, $112 \mathrm{pp}$.

Steel, R. G. \& Torrie, J. H., 1960. Principles and procedures of statistics. McGraw-Hill, New York, $481 \mathrm{pp}$.

Steemann Nielsen, E., 1952. The use of radio-active carbon $\left({ }^{14} \mathrm{C}\right)$ for measuring organic production in the sea. J. Cons. perm. int. Explor. Mer. 18, 117-140.

- 1960. Dark fixation of $\mathrm{CO}_{2}$ and measurements of organic productivity. With remarks on chemosynthesis. Physiologia Pl. 13, 348-357.

-1965 . On the determination of the radioactivity in ${ }^{14} \mathrm{C}$ - ampoules for measuring primary production. Limnol Oceanogr. 10, 247-252.

Straaten, L. M. J. U. van, 1954. Composition and structure of recent marine sediments in the Netherlands. Leid. geol. Meded. 19, 1-110.

Strickland, J. D. H. \& Parsons, T. R., 1972. A practical handbook of seawater analysis. Bull. Fish. Res. Bd Can. 167, 1-310.

Stugren, B., 1972. Grundlagen der allgemeinen Okologie. VEB Fischer, Jena, 223 pp.

Swan Wooster Engineering Co. Ltd., 1967. Roberts Bank and Sturgeon Bank harbour study. Topographic maps. Prep. by Lockwood Survey Corp., National Harbours Board, Vancouver, B. C. 
Tabata, S., Giovando, L. F. \& Devlin, D., 1971. Current velocities in the vicinity of the Greater Vancouver sewerage and drainage districts Iona Island outfall - 1968. Tech. Rep. Fish. Res. Bd Can. 263, 1-86.

Taylor, W. R. \& Gebelein, C. D., 1966. Plant pigment and light penetration in intertidal sediments. Helgoländer wiss. Meeresunters. 13, 229-237.

Tebble, N., 1966. British bivalve seashells. British Mus. Nat. Hist., London, 212 pp.

Thamdrup, H. M., 1935. Beiträge zur Okologie der Wattenfauna auf experimenteller Grundlage. Meddr Komn Danm. Fisk-og Havunders. 10, 1-125.

Thienemann, A., 1941. Leben und Umwelt. Bios 12, 1-122.

Tietjen, J. H., 1968. Chlorophyll and phaeo-pigments in estuarine sediments. Limnol. Oceanogr. 13, 189-192.

Trask, P. D., 1930. Mechanical analysis of sediments by centrifuge. Econ. Geol. 25, 581-599.

Tulkki, P., 1968. Effects of pollution on the benthos of Gotenburg. Helgoländer wiss. Meeresunters. 17, 209-215.

- 1969. Die Bodentiere als Indikatororganismen für Verunnreinigung in einem Brackwassergebiet. Limnologica 7, 79-85.

Ushakov, P. V., 1965. Polychaeta of the far-eastern seas of the U.S.S.R. Israel Program Scient. Transl., Jerusalem, $448 \mathrm{pp}$.

Wass, M. L., 1967. Biological and physiological basis of indicator organisms and communities. II. Indicators of pollution. In: Pollution and marine ecology. Ed. by T. A. Olsen \& F. J. Burgess, Wiley-Interscience, London, 261-283.

Watling, L., Leathem, W., Kinner, P., Wethe, C. \& Maurer, D., 1974. Evaluation of sludge dumping off Delaware Bay. Mar. Pollut. Bull. 5, 39-42.

Weber, E., 1967. Grundriß der biologischen Statistik. Fischer, Stuttgart, 674 pp.

Wilhelmi, J., 1915 a. Leitsätze für die Einleitung von Abwässern in das Meer. Wass. Abwass. 9, 513-517.

- 1915 b. Untersuchungen, besonders in biologisch-mikroskopischer Hinsicht, über die Abwasserbeseitigung von Küstenorten. Mitt. Landesanst. Wass.-Hyg. 20, 114-194.

Wilhm, J. L., 1967. Comparison of some diversity indices applied to populations of benthic macroinvertebrates in a stream receiving organic wastes. J. Wat. Pollut. Control Fed. 39, 1673-1683.

Wohlenberg, E., 1937. Die Wattenlebensgemeinschaften im Königshafen von Sylt. Helgoländer wiss. Meeresunters. 1, 1-92.

Young, P. H., 1964. Some effects of sewer effluents on marine life. Calif. Fish Game 50, 33-41.

Ziegelmeier, E., 1957. Die Muscheln (Bivalvia) der deutschen Meeresgebiete. Helgoländer wiss. Meeresunters. 6, 1-56. 